\title{
Emergent spacetime and holographic CFTs
}

\author{
Sheer EI-Showk ${ }^{a, b}$ and Kyriakos Papadodimas ${ }^{c}$ \\ ${ }^{a}$ Institut de Physique Theeorique, \\ CEA Saclay, CNRS URA 2306, F-91191 Gif-sur-Yvette, France \\ ${ }^{b}$ Institute for Theoretical Physics University of Amsterdam, \\ Science Park 904, Postbus 94485, 1090 GL Amsterdam, The Netherlands \\ ${ }^{c}$ Theory Group, Physics Department, CERN, \\ CH-1211 Geneva 23, Switzerland \\ E-mail: sheer.el-showk@cea.fr, kyriakos.papadodimas@cern.ch
}

ABSTRACT: We discuss universal properties of conformal field theories with holographic duals. A central feature of these theories is the existence of a low-lying sector of operators whose correlators factorize. We demonstrate that factorization can only hold in the large central charge limit. Using conformal invariance and factorization we argue that these operators are naturally represented as fields in AdS as this makes the underlying linearity of the system manifest. In this class of CFTs the solution of the conformal bootstrap conditions can be naturally organized in structures which coincide with Witten diagrams in the bulk. The large value of the central charge suggests that the theory must include a large number of new operators not captured by the factorized sector. Consequently we may think of the AdS hologram as an effective representation of a small sector of the CFT, which is embedded inside a much larger Hilbert space corresponding to the black hole microstates.

KEYwords: Gauge-gravity correspondence, AdS-CFT Correspondence, Conformal Field Models in String Theory, 1/N Expansion

ARXIV EPRINT: 1101.4163 


\section{Contents}

1 Introduction 1

2 Main picture: conditions for holographic CFTs 3

2.1 Some comments 6

2.2 Examples 8

$\begin{array}{lll}2.3 & \text { Counter-examples } & 13\end{array}$

2.4 Do we need gauge-symmetry? 14

3 Consistency requirements for a CFT $\quad 15$

$\begin{array}{ll}3.1 \text { Conformal bootstrap } & 16\end{array}$

$\begin{array}{ll}3.2 & \text { Modular invariance and consistency at finite temperature } \\ 3.38\end{array}$

3.3 "Cardy formula" and higher dimensional CFTs 22

4 Generalized Free CFTs and holography $\quad 22$

4.1 Generalized free fields: factorization for $\Delta>\frac{d-2}{2} \quad 23$

4.2 Conformal bootstrap for generalized free fields and multiparticle spectrum 24

4.3 Partition functions of generalized free fields 26

4.4 Decoupling of the stress tensor and the need for large $c \quad 28$

4.5 Gravitational collapse and "deconfinement" 29

4.6 Minimal generalized free CFT and gas of gravitons 31

$\begin{array}{lll}4.7 & \text { Summary } 33\end{array}$

5 Emergence of the holographic dimension 33

5.1 Why this is not just group theory $\quad 35$

$\begin{array}{lll}5.2 & \text { Operators with spin } & 35\end{array}$

5.3 Comments on bulk observables and background independence 36

$\begin{array}{llr}6 & \text { Including interactions } & \mathbf{3 7}\end{array}$

$\begin{array}{ll}\text { 6.1 Scaling of correlators with } c & 38\end{array}$

6.2 Conformal bootstrap in perturbation theory and holographic interactions $\quad 39$

6.3 CFT interpretation of Witten diagrams: "dressed" conformal blocks 41

6.4 Summary: CFT interpretation of the basic exchange Witten diagram 45

6.5 Dressing the OPE 46

6.6 Conformal bootstrap for higher $n$-point functions 47

6.7 Generalized free CFTs and expansion in Witten diagrams 50

7 The CFT at finite temperature $\quad \mathbf{5 1}$

7.1 Factorization requires an infinite number of degrees of freedom 51

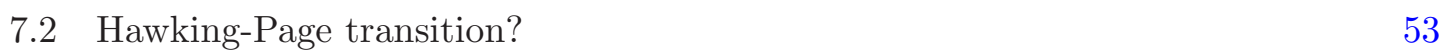

7.3 Extending the "Cardy regime"? 54 
$\begin{array}{ll}\text { A Conformal partial waves } & 57\end{array}$

B CPW expansion of Witten diagrams $\quad \mathbf{5 8}$

$\begin{array}{ll}\text { B.1 Contact Witten diagram } & 58\end{array}$

$\begin{array}{ll}\text { B.2 Exchange Witten diagram } & 60\end{array}$

C Central charges in higher dimensional CFTs 62

\section{Introduction}

One of the most important ideas considered in the last decades is the possibility that space and time may be emergent concepts. In string theory we know examples of dualities in which spacetime, or at least some of its dimensions can be reconstructed from certain underlying quantum systems. ${ }^{1}$ Besides refining the technical aspects of such dualities, it is important to identify the general principles governing the universality class of quantum systems from which a macroscopic semi-classical spacetime can emerge.

In this paper we want to revisit this question in a simplified context. We will focus on the emergent nature of gravitational theories in anti-de Sitter space. After the discovery of the AdS/CFT correspondence [2-4] it has been understood that such theories are "dual" to conformal field theories living in lower dimensions. At the moment it is only the field theory side of these dualities which can be non-perturbatively defined. From the field theory point of view the bulk anti-de Sitter spacetime, with its gravitational interactions, is an emergent concept. Is this phenomenon isolated to very special conformal field theories or is it generic? And if so, what are the general principles behind the emergence of a "dual AdS spacetime"? We propose to explore these issues by posing the following question:

Which conformal field theories have a holographic description and why?

To address this question we will review properties of well-known CFTs with holographic duals and attempt to recast them in a general language that abstracts away the specifics of the given theory. The philosophy of our approach will be the following: we want to explore why and how, for a certain class of CFTs, an observer unaware of the AdS/CFT correspondence, would naturally end-up "rediscovering" it. That is, using only the consistency of the CFT, we will try to understand why it would be natural to formulate it as an effective gravitational theory in AdS.

The general approach of our paper is closely related to various other works which appeared in the past [5-9]. Similar questions have also been addressed recently in [10-13] on which we heavily base some of our discussions. The emergence of gravity has also been

\footnotetext{
${ }^{1}$ See $[1]$ for a recent review.
} 
recently addressed in [14] from another point of view and also in [15, 16]. Many of the statements in this paper are well known to the experts and we do not have many new technical results to report. Nonetheless we hope that our presentation may be useful.

To summarize the main points, in this paper we argue that a CFT is naturally described holographically in AdS space if it has the following basic properties:

i) It has large central charge i.e. many degrees of freedom.

ii) It has a small number of operators of low conformal dimension.

iii) The correlators of the low lying operators factorize. ${ }^{2}$

Intuitively these conditions mean that we should be looking for CFTs with a large number of degrees of freedom i.e. many fundamental fields, but few weakly coupled light ${ }^{3}$ operators. $^{2}$ We will try to argue that in theories with these properties the effective interactions of the low-lying operators are naturally described in terms of a dual gravitational theory in antide Sitter space. These conditions, as well as the meaning of the holographic dual theory, will be made more precise in the rest of the paper.

Conditions i), ii), iii) are automatically satisfied for large $N$ gauge theories in the 't Hooft limit: for these theories the central charge scales like $N^{2}$, the low-lying operators are the gauge invariant combinations of traces (whose number is small i.e. $N$-independent) and factorization of single trace operators is guaranteed by the large $N$ 't Hooft factorization. We want to argue that the aforementioned conditions are the central universal features of CFTs with holographic duals. We expect that any CFT with these properties, even if it is not a standard gauge theory, will have a natural holographic description. From this point of view it would seem that the main role of gauge invariance for holography of large $N$ gauge theories is that it is an efficient way to engineer quantum field theories in which conditions i), ii), iii) are met.

In the rest of the paper we start from the bottom-up. We start by considering CFTs which contain operators whose correlators factorize. Such operators have certain features of ordinary free fields, though they do not obey linear equations of motion. We will refer to them as "generalized free fields". By uplifting these operators to one dimension higher they can be extended to ordinary free fields, thus making the underlying linearity of the system manifest. In this sense a CFT observer would have a reason to introduce one extra dimension in exchange for having linear equations of motion.

When introducing small interactions around such generalized free fields we have to make sure that they satisfy the "conformal bootstrap" conditions. We argue it is natural to reorganize the perturbed CFT correlators in terms of algebraic structures which coincide with what we would normally call "Witten diagrams" in the bulk. For this we study in some detail the relation between Conformal Partial Waves (CPWs) on the boundary and Witten diagrams in the bulk. We will try to argue that a physicist, unaware of AdS/CFT, who would try to solve the conformal bootstrap conditions around a "generalized free CFT"

\footnotetext{
${ }^{2}$ As we will explain later, condition iii) requires i), but not the other way around.

${ }^{3}$ In the sense of having small conformal dimension.
} 
would reorganize the solution in terms of objects which look like the Witten diagrams and might thus be led to the formulation of a gravitational theory in AdS.

Finally we will see that such generalized free CFTs are not fully consistent by themselves and can only be understood as being small sectors of much bigger underlying conformal field theories. The reason is that in order to have factorization of correlators it is necessary for the central charge of the CFT to be large. This implies that at large conformal dimension the theory must have a large number of operators that do not appear in the naive spectrum of generalized free fields. From the bulk point of view these operators correspond to the black hole microstates.

The approach of our paper seems to be more consistent with the idea that the bulk is the effective description of a small semi-independent sector of low-lying operators in the conformal field theory i.e. of the "confined phase" in the language of gauge theories. According to the logic followed in our presentation it seems that there would be no reason for operators with large conformal dimension, i.e. those corresponding to black hole microstates, to have representatives in the bulk in terms of local fields (though they might be represented as states living, in a sense, on black hole horizons).

The plan of the paper is as follows. In section 2 we give an overview of the main ideas of the paper, including some motivational examples, eschewing any technical details. Section 3 provides some background on standard CFT consistency conditions as well as introducing constraints following from consistency of the thermal theory. In section 4 we introduce the notion of a Generalized Free Field and study the structure of CFTs composed of such operators; in particular it is argued that such theories cannot define consistent local CFTs unless augmented by additional operators. By recasting them in a higher dimensional language where their linear structure is manifest it is argued in section 5 that such generalized free CFTs are, in a sense, inherently holographic. This is further developed in section 6 where perturbations around such theories are studied. The latter are most naturally organized in a structure that mimics Witten diagrams in a putative AdS bulk. In section 7 we return to the spectrum and argue that factorization implies a divergent entropy density. The relationship to Cardy's formula in two dimensions and the extended nature of the "Cardy regime" in AdS duals is also discussed. Section 8 closes with some discussion and proposals for future directions. A series of appendices contain technical background and results. Appendix A provides background on the Conformal Partial Wave decomposition of CFT correlation functions while appendix B includes a computation of the coefficients of such an expansion applied to Witten diagrams. Appendix $\mathrm{C}$ reviews the different notions of central charges in $d>2$.

\section{Main picture: conditions for holographic CFTs}

In this section we summarize the main picture without going into technical details. A CFT is by itself well-defined even at strong coupling (for example by simulating it on a lattice) and in principle there is no need to invoke a holographic dual description. The reason that for some CFTs we do it nevertheless is that introducing the "dual spacetime" makes the description of the theory (or perhaps a sector of the theory) simpler. In general a given 
physical theory may admit more than one equivalent mathematical description. We do not have a sharp quantitative criterion to decide which of them is the "simplest" one, however we will follow the naive intuition that if a physical theory admits a description in terms of a small number of weakly interacting local fields living on a spacetime then this description is a natural one to use.

To proceed we first have to explain what kind of dual gravitational theories we have in mind. At the moment an independent definition of what is meant by "quantum gravity in AdS" in not available. We only know what semiclassical gravity or perturbative string theory means, which implies that the Planck length must be taken to be much smaller than the effective curvature radius of the AdS space. So we will focus on analyzing CFTs which can potentially describe weakly coupled dual holographic theories (in the sense of small $g_{s}$, not necessarily small curvature). These holographic theories may look like two-derivative gravity, like weakly coupled string theory at high (string) curvatures, M-theory, higher spin gravity or something more exotic. Since we want to be general, we will take the following as the minimal requirement for a holographic bulk theory:

We will assume that as we take the weak coupling limit ${ }^{4}$ in the bulk, the number of light modes stays finite.

By this we mean that the number of modes below any given (and fixed) energy scale remains finite as we send the coupling, measured by the ratio of the Planck to the AdS length scale, to zero. This condition is satisfied in all known examples of AdS/CFT with a weak coupling limit (again, in the sense of small $g_{s}$ ) and if violated it seems unlikely that any useful notion of spacetime, classical or stringy, would emerge.

Let us translate these statements into expectations for the dual CFT. First of all, since we want to consider theories with a weak coupling limit we should be looking for families of CFTs characterized by some parameter ${ }^{5} g$, with the property that as $g \rightarrow 0$ we reach the free limit in the bulk. In standard large $N$ gauge theories this parameter would be $g \sim \frac{1}{N}$. According to the condition that we mentioned above, we will assume that in this limit the CFT has a finite number of operators of low conformal dimension. ${ }^{6}$ Moreover we will assume that in the limit $g \rightarrow 0$ the correlators of these low-lying operators factorize (in a sense that will be made more precise later). We believe that this is the most crucial element of a CFT with a holographic dual.

Note that while this may sound innocuous it is a rather non-trivial constraint: the basic axioms of a CFT imply that such a set of factorized operators can only arise as a small subsector of a much bigger conformal field theory. The basic reason for this is that the assumption of factorization of correlators conflicts with a universal interaction in conformal field theories, the one mediated by the exchange of the stress-energy tensor between operators. The coupling of the stress energy tensor to other conformal primaries

\footnotetext{
${ }^{4}$ i.e. the classical limit, not necessarily a small curvature limit.

${ }^{5}$ The parameter $g$ does not have to be a continuous variable; in particular it may not be an ordinary coupling constant. For example, it may be a discrete label in a sequence of theories.

${ }^{6}$ To be more precise, we assume that for any given $\Delta$ the number of operators with dimension lower that $\Delta$ remains finite as $g \rightarrow 0$. It is important to take the limit in this way, that is first fix $\Delta$ and then send $g \rightarrow 0$.
} 


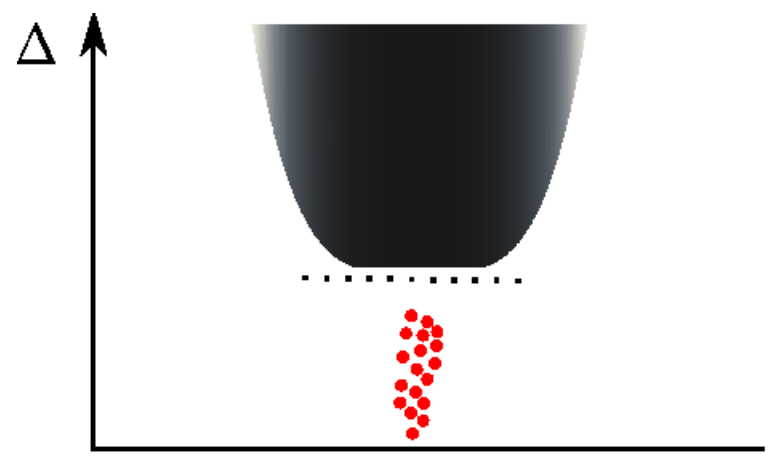

Figure 1. Typical spectrum of CFTs with holographic duals. The shaded part at large conformal dimension represents a large (c-dependent) number of heavy operators, which correspond to black hole microstates. The red dots represent a small number ( $c$-independent) light operators, whose correlators factorize. These operators are represented holographically as free (or weakly interacting) fields in AdS. This is only a cartoon and the spectrum at intermediate values of conformal dimension may have complicated form.

is fixed by the Ward identities and, as we will see, the only way to have it decoupled (which is necessary to have factorization) is that, as $g \rightarrow 0$ the central charge ${ }^{7} c$ of the CFT goes to infinity. For simplicity, and to be consistent with the case of large $N$ gauge theories, in the rest of the paper we will make the identification $g=\frac{1}{\sqrt{c}}$. The fact that the central charge goes to infinity means that the number of degrees of freedom in the theory is large. Since we assumed that we have a finite number of operators at low conformal dimension, this large number of degrees of freedom has to emerge at large conformal dimension.

Now we can phrase our criterion more precisely. We are looking for CFTs with large central charge $c$ with the property that in the $c \rightarrow \infty$ limit their spectrum has the following structure: at conformal dimensions of order $\mathcal{O}(1)$, as far as the $c$-scaling is concerned, the number of operators remains finite, while above a certain conformal dimension $\Delta_{*}$ (which scales to infinity as $c \rightarrow \infty$ ) we have a $c$-dependent proliferation of states. ${ }^{8}$ The theory has a small low-lying sector separated from the huge number of operators with large conformal dimension of order $\Delta_{*}$ and higher as depicted in figure 1 .

In the bulk the quantity $g$ plays the role of $\hbar$, hence in the limit $c \rightarrow \infty$ we have $\hbar \rightarrow 0$. The low-lying operators of the CFT are dual to the (perturbative) supergravity/closed string modes whose numbers remains finite as we send $\hbar \rightarrow 0$. On the other hand the large number of states of conformal dimension above $\Delta_{*}$ corresponds to black hole microstates whose degeneracy blows up in the classical limit $\hbar \rightarrow 0$ as can be seen from the BekensteinHawking entropy formula.

Moreover we assume that in the limit $c \rightarrow \infty$ the low-lying operators factorize i.e. they become generalized free fields. ${ }^{9}$ As we will try to argue in the rest of the paper, the effective

\footnotetext{
${ }^{7}$ Defined by the 2-point function of the stress-energy tensor.

${ }^{8}$ This is to be understood as a qualitative statement, since the change of degeneracies from $\mathcal{O}(1)$ to $\mathcal{O}(c)$ does not have to be sharp, there may be intermediate regimes.

${ }^{9}$ While we use the term "field" for these operators it should be clear that they are not "fundamental" fields of the Lagrangian over which one should path integrate.
} 
dynamics of the low-lying generalized free fields can be most naturally represented in an AdS space with semi-local ${ }^{10}$ interactions. From this point of view the hologram seems to be an effective representation of a small part of the Hilbert space of the CFT (a similar point of view is espoused in [12]). Of course according to the strong version of the AdS/CFT correspondence, it may become possible in the future to define the bulk side independently, including all of the $\mathcal{O}(c)$ degrees of freedom.

\section{$2.1 \quad$ Some comments}

In this subsection we mention some clarifications.

1. From the known examples of AdS/CFT it is clear that the holographic representation of a CFT can have various degrees of complexity. The simplest case is the one where the bulk theory is classical gravity, possibly with a few other light fields, on a background of the form $\operatorname{AdS} \times M$, where $M$ is some internal manifold whose size is large compared to the Planck and other UV scales (such as the string scale). These theories are the ones where there is a parametric separation between the mass of fields with spin up to two and those with higher spin, and the relevant conditions in the CFT were discussed in [10]. A next level of complexity corresponds to theories with a significantly larger number of light fields in the bulk, in which the aforementioned separation between spins does not exist. Examples of this kind are higher-spin gravity or classical string theory on highly curved backgrounds. In the latter class we have, for example, the $\mathcal{N}=4 \mathrm{SYM}$ at large $N$ and finite/small 't Hooft coupling $\lambda$. Finally there may exist even more exotic bulk theories, such as those dual to CFTs at finite $N$ - i.e. fully quantum gravity, or dual to CFTs where there is no qualitative separation in the degeneracies of the spectrum between small and large conformal dimensions (see "counter-examples" subsection below).

When we pose the question "which CFTs are holographic?" we have to specify what kind of bulk theories we are referring to. In this paper we decided to focus on the universality class of bulk theories which are semi-classical but may be highly curved/stringy (so, for example, we would include the $\mathcal{N}=4 \mathrm{SYM}$ at small 't Hooft coupling, but not at finite $N$ ). The reason for doing so is because this class of theories seems general enough to include various perturbative string theory backgrounds as well as some of the more general models mentioned in section 2.2. At the same time we did not want to be too general, since, had we gone into the quantum gravity regime (finite central charge), we would have to deal with the problem of defining (independently of the CFT) what we mean by quantum gravity in an AdS space of Planckian size. ${ }^{11}$

\footnotetext{
${ }^{10}$ By this we mean that the AdS theory is not necessarily a local effective field theory with a small number of light fields. It could, for example, be a highly curved (but weakly coupled) string theory.

${ }^{11} \mathrm{By}$ this we do not mean to claim that there are no such bulk theories, as this would be inconsistent with the strong version of AdS/CFT, but rather that we do not know any such theory yet and hence it is not possible for us to say anything concrete about what to expect from such theories.
} 
2. The "jump" in the degeneracies of the spectrum that we refer to should be distinguished from the gap discussed in [10]. In that paper the gap was between operators corresponding to supergravity fields and those dual to "closed string states" i.e. it was a gap within the low-lying generalized free field sector, from our point of view. The change in the qualitative properties of the spectrum ${ }^{12}$ that we are talking about is between operators with conformal dimension of order $\mathcal{O}(1)$ (dual to perturbative gravitational and stringy states) and operators of dimension $\mathcal{O}(c)$ which are dual to black hole-like states i.e. between the generalized free field sector and the other operators in the CFT. ${ }^{13}$

3. The generalized free fields have been discussed before in the literature, however we should emphasize that our point of view is quite different. We claim that such theories cannot be considered as fundamental CFTs, but only as limits of certain small sectors of much larger CFTs. ${ }^{14}$

4. The coupling $g=1 / \sqrt{c}$ is a universal coupling in conformal perturbation theory mediated by the exchange of the stress-energy tensor, as it follows strictly from conformal invariance and the Ward identities. One might then argue that the additional "black hole microstates" with $c$-dependent masses and degeneracies are, in some sense, the associated non-perturbative states. As generalized free theories do not have a local $d$-dimensional Lagrangian description this analogy can only be made precise in the bulk dual (where it is manifest).

5. In large $N$ gauge theories the conditions i), ii), iii) are automatically satisfied because of two important physical effects: confinement/deconfinement (which guarantees the right qualitative properties of the spectrum) and the 't Hooft large $N$ factorization. But as we mentioned in the introduction it is perhaps true that these two properties, spectrum and factorization, are of more fundamental importance for holography than gauge invariance itself.

6. Our approach has an important limitation: we will discuss the emergence of the bulk from the CFT but the structure that we will describe will be an effective theory in AdS, with potentially a very large (though $c$-independent) number of fields. The missing element is finding the "organizing principle" behind this effective AdS theory. For example if we were to follow our logic for the $\mathcal{N}=4 \mathrm{SYM}$ at weak 't Hooft coupling we would end up with an AdS theory with a large number of fields (corresponding to the stringy modes, whose number grows exponentially with conformal dimension), but without knowing that there is an underlying worldsheet string theory

\footnotetext{
${ }^{12}$ We would like to thank E. Kiritsis for helpful comments.

${ }^{13}$ Of course the two statements are not in contradiction since the goal of [10] was to identify CFTs with local gravity duals and not more general stringy ones. Also the property of the spectrum that we are talking about is presumably assumed in [10], though not explicitly discussed.

${ }^{14}$ Here we are referring to works done in the $60 \mathrm{~s}$ and $70 \mathrm{~s}$ in the context of axiomatic QFT (see for example [17] and also more recently to the work on "algebraic holography", for instance [18, 19], where generalized free fields are also used, but seen as fundamental theories.
} 
governing these fields. In this sense the approach initiated by R. Gopakumar [20-23] and also $[24,25]$ is more ambitious, since it attempts a direct reconstruction of the bulk worldsheet theory from the boundary correlators.

7. On the other hand it is possible that there exist CFTs with effective holographic duals which do not correspond to string theories, in the sense of perturbative worldsheet theories (examples are M-theory backgrounds or the duals of $O(N)$ models [26]). For such CFTs it is not clear what kind of structure we would expect in the bulk so we restrict ourselves to constructing an effective AdS theory.

8. It would be useful to find a precise way to quantify, from the CFT point of view, the extent to which the dual AdS theory is local. One criterion seems to be the number of single-particle operators at low conformal dimension. It is natural to expect that the fewer operators there are at low conformal dimension, the sharper the notion of locality and of classical geometry in AdS will be. This can be seen by considering three different classes of examples with increasing complexity: theories with classical gravity duals, higher spin gravity theories and highly curved (weakly coupled) string theories. The notion of geometry is most clear in the first case, it becomes more complicated in the second, and in the third the only notion of spacetime is the one provided by the worldsheet conformal field theory (i.e. spacetime is "stringy"). Correspondingly the number ${ }^{15}$ of single trace operators is small in the first case, where as emphasized in [10] there is a parametric separation in the dimensions of operators with spin 2 and higher, larger in the second and exponentially growing with conformal dimension in the third. There may of course exist other possible forms of the spectrum in holographic CFTs that have not been discovered yet.

\subsection{Examples}

To motivate and provide context for the rest of our discussion let us consider the general qualitative features of CFTs known to admit AdS duals. Most of the known examples fall broadly into four main categories: 1) large $N$ gauge theories, 2) large $N$ symmetric orbifolds, 3) large $N$ vector $O(N)$ models and 4) particular $\sigma$-models on targets spaces of parametrically high-dimension (i.e. the MSW CFT [27]).

Large $N$ gauge theories. Large $N$ gauge theories in the 't Hooft limit are the canonical example of quantum field theories with holographic duals. This was already suggested by the observation of 't Hooft that the $1 / N$ expansion in gauge theories can be interpreted as a genus expansion of a dual string theory [28]. After the AdS/CFT correspondence [2] the $\mathcal{N}=4 \mathrm{SYM}$ at large $N$ became the best studied example of a theory with a gravitational dual, along with a very large number of other holographic gauge theories analyzed in the last decade.

Let us see how large $N$ gauge theories satisfy the criteria i), ii), iii) that we presented. For simplicity we will only consider the case where the gauge theories are conformal and

\footnotetext{
${ }^{15}$ And growth with conformal dimension.
} 
we will also restrict ourselves to gauge theories with a weak coupling limit in their moduli space. ${ }^{16}$

First we study the spectrum at low conformal dimension. ${ }^{17}$ If we consider the $N \rightarrow$ $\infty$ limit then according to arguments of 't Hooft the theory simplifies considerably with correlators of gauge-invariant operators factorizing. Such factorization implies that the spectrum of the theory should enjoy a particularly simple form and at weak coupling we can compute the spectrum explicitly [29-31]. The requirement of gauge invariance restricts the spectrum of local operators to be composed of traces, thus suppressing the dependence on $N$. Single-trace operators can be constructed from the fundamental fields $\Phi_{i}$ of the gauge theory as $\operatorname{Tr}\left[\Phi_{i_{1}} \ldots \Phi_{i_{n}}\right]$ with $n \ll N$, whose degeneracy depends only on $n$. Due to large $N$ factorization the spectrum has the form of a freely generated Fock space of multi-trace operators, where the basic excitations are the single-trace operators.

To summarize, we argued that at low conformal dimension the theory has a small number of operators (i.e. their degeneracy does not scale with $N$ ), whose correlators factorize due to large $N$ combinatorics. Hence the conditions ii) and iii) are satisfied.

When, however, the length and number of traces become large (i.e. when either starts to scale with $N$ ), trace relations correct the spectrum, introducing an $N$-dependence. This is an essential feature since otherwise the finite temperature partition function would encounter a Hagedorn-type divergence at temperatures of order 1, caused by the exponential growth of single-trace operators as a function of their conformal dimension. Moreover, even if, for some reason, there was no exponential growth in the degeneracy of single-trace operators, and if the degeneracy were to continue to scale naively as a multiparticle gas of single-trace operators, then the high temperature behaviour of this theory would be inconsistent with conformal invariance (see section 4.3). Hence factorization should break down when the conformal dimension of operators becomes large (that is, $N$ dependent). Despite the very gauge-theoretic nature of this structure we will see that something similar occurs quite generally in any large $c$ CFT satisfying assumptions ii) and iii).

Let us now consider what happens at large conformal dimension. Although the central charge is not a unique notion in higher dimensional CFTs, the various notions of central charge (see e.g. Section 3.3 and appendix C) are all proportional to $N^{2}$ for gauge theories. Intuitively these $N^{2}$ degrees of freedom correspond to the $N^{2}$ components of the fundamental fields of the theory. This is a reliable estimate if the theory admits a weak coupling limit. ${ }^{18}$ We thus expect that the entropy density at very high temperatures will grow like $s \sim N^{2} T^{d-1}$ which is consistent with condition (i). The spectrum of operators of the gauge theory is related, via the state-operator map, to the spectrum of states of the CFT on $\mathbf{S}^{d-1} \times \mathbf{R}$. The high temperature entropy density mentioned above implies that

\footnotetext{
${ }^{16}$ We will also assume that the number of flavor fields does not scale with $N$, otherwise the scaling of various quantities mentioned below must be modified. We thank E. Kiritsis for comments about these issues.

${ }^{17}$ I.e. conformal dimension of order 1.

${ }^{18}$ Notice however that this is not true beyond the 't Hooft limit, for theories which are strongly coupled. For example in the ABJM theory [32] at $k=1$, while the number of fundamental fields of the theory scales like $N^{2}$ the prediction from gravity is that the entropy density scales like $N^{3 / 2}$. In this case the strong coupling dynamics invalidates the naive free-field intuition about the number of degrees of freedom [33].
} 
the entropy of operators of conformal dimension $\Delta$ grows like $S \sim N^{2 / d} \Delta^{(d-1) / d}$. This indicates a very large number of operators of large conformal dimension as depicted in figure 1.

These two regimes (of small and large conformal dimension) correspond to different phases of the theory. When we consider large $N$ gauge theories in the canonical ensemble we encounter a phase transition associated with deconfinement; the free Fock space states (and their interacting generalizations) dominate in the low temperature confining phase (dual to free gravitons in AdS) and the high temperature deconfined quark-gluon plasma phase is associated with heavy black hole microstates with an $N$-dependent entropy. At strong coupling in the bulk this is the Hawking-Page phase transition [34], which was first related to deconfinement in the gauge theory in [4]. In general large $N$ gauge theories a range of intermediate behaviours is possible [31] encapsulating various other phase transitions (e.g. an intermediate stringy regime with Hagedorn behaviour) but for our purposes the picture presented here is sufficient.

Symmetric orbifolds. A second class of theories with holographic duals are large $N$ symmetric orbifold CFTs in two dimensions. Perhaps the best studied examples are 2-d $\sigma$-models with symmetric orbifold target spaces $\left(\mathcal{M}^{N} / S_{N}\right.$ with $\mathcal{M}=K 3$ or $T^{4}$ as the best known examples), which are realized on bound states of D1 and D5 branes in IIB string theory [35]. More generally starting with any two dimensional conformal field theory $\mathrm{CFT}_{a}$ we can construct the symmetric orbifold $\mathrm{CFT}_{N} \equiv\left(\mathrm{CFT}_{a}\right)^{N} / S_{N}$. At large $N$ the theory $\mathrm{CFT}_{N}$ satisfies all the criteria that we listed and thus should have some sort of holographic dual (most likely stringy).

Let us see how the conditions i), ii), iii) are satisfied in this case. While the central charge of the theory $\mathrm{CFT}_{N}$ is $N$ times the central charge of $\mathrm{CFT}_{a}$ the low-lying spectrum does not enjoy the naive $N$ dependence one might expect from a theory with order $N$ degrees of freedom. This is because the orbifold projection restricts the spectrum only to symmetrized states so e.g. if $\mathrm{CFT}_{a}$ is a $\sigma$-model on $\mathcal{M}$ the supersymmetric zero mode wavefunctions are not $N$ copies of the cohomology of $\mathcal{M}$ but a single copy. Thus at low conformal dimension the degeneracy of operators is small, in the sense that it does not grow with $N{ }^{19}$

The correlators also enjoy a large $N$ expansion implying factorization at $N \rightarrow \infty[36-$ 38 ] and consequently we expect a simple structure for the multi-particle spectrum. These low-lying states are separated from the heavy ones by a phase transition. The phase transition in this system has a rather different origin from that of gauge theories. Here it is associated with the appearance of long strings [39]. Orbifold CFTs have twisted sectors coming from long strings with winding number $w$. These strings can be described by inserting twist operators with $\Delta \sim w$. Because these strings are long they have a lower mass gap and hence larger degeneracy at fixed temperature than a shorter string. The degeneracy of the short strings is $N$-independent since we must symmetrize over the strings whereas a single string with $w \sim N$ (the max winding number) feels an "effective"

\footnotetext{
${ }^{19}$ For supersymmetric CFTs the statements about the spectrum have to be understood in the NS sector which is dual to $\mathrm{AdS}_{3}$ in global coordinates.
} 
temperature $T \gg 1$ even if the real temperature is order one because the mass gap on this string goes as $1 / N$. Hence Cardy's formula can be used, giving an entropy density $s \sim N T$. Thus at a temperature of order one ${ }^{20}$ the increased entropy of the long string overcomes their energy cost and there is a phase transition from a short string to a long string phase. This implies that the degeneracy of operators with large conformal dimension does indeed scale with $c \sim N$. Note that while the qualitative structure is similar to a gauge theory the actual mechanism for the phase transition is quite different.

$\boldsymbol{O}(N)$ \& related vector models. Another example of a CFT which satisfies our criteria i), ii), iii) is the $O(N)$ vector model. The central charge of the CFT grows with $N$, while at low conformal dimension the number of local operators which are $O(N)$ invariant is $N$-independent i.e. small. Large $N$ factorization of "single-particle" correlators also holds.

It is believed [26] that this theory (perhaps appropriately gauged) is dual to a higher spin gravitational theory in anti-de Sitter space [40-42]. See [43-48] for related work. Further evidence for this conjecture has been provided recently with the computation of 3-point functions on both sides of the duality [49] demonstrating that they agree.

The example of the $O(N)$ model is particularly interesting because the bulk theory is not classical gravity, in the sense that it contains an infinite number of fields and there is no parametric separation between the graviton and the higher spin fields, while at the same time it is not a string theory. ${ }^{21}$ This suggests that the landscape of holographic CFTs may contain various exotic possibilities.

Recently [50] considered coset WZW models and showed that these CFTs provide a two-dimensional analog of vector $O(N)$ models. In particular they are dual to higher spin theories on $\mathrm{AdS}_{3}$. Moreover, such CFTs come in families parametrized by a coupling constant $0 \leq \lambda \leq 1$ analogous to the 't Hooft coupling of gauge theories. Unlike gauge theories, however, this coupling is bounded from above so the associated bulk geometry is never a standard gravity dual. The authors of [50] nonetheless present evidence for a complete equivalence of the spectrum in the $N \rightarrow \infty$ limit. See also [51]. This example suggests that the notion of a bulk geometry as providing an effective description of a CFT at large $c$ is not restricted to "string" bulks with a weak-coupling gravity regime. Rather, as we will argue, a large $c$ CFT is naturally described by an AdS theory in one higher dimension with a possibly large, but $c$-independent, number of (approximately free) fields.

The MSW CFT. Perhaps the most poorly understood example of a theory with a known gravitational dual is the $\mathcal{N}=(0,4)$ CFT described in [27] (see also [52]) as the lowenergy dimensional reduction (to $1+1 \mathrm{~d}$ ) of the theory on a smooth M5-brane wrapping $\mathcal{M} \times S^{1} \times \mathcal{R}_{t}^{1}$ with $\mathcal{M}$ a holomorphic divisor (four-cycle) in a CY $X$ with a Poincare dual $[p] \in H^{2}(X), S^{1}$ the M-theory circle of radius $R$, and $\mathcal{R}_{t}^{1}$ time.

\footnotetext{
${ }^{20}$ More precisely it occurs at temperature $T=1 /(2 \pi)$ which corresponds to a torus which is invariant under the modular transformation $\tau \rightarrow-\frac{1}{\tau}$. Here we assume that the CFT is defined on a spatial circle of length $2 \pi$.

${ }^{21}$ At least not a conventional one, for example the growth of single-trace operators in the CFT does not exhibit a Hagedorn (exponential) growth with conformal dimension, unlike what happens in adjoint-valued gauge theories.
} 
While little is known about this theory it is clear from the M5-decoupling limit that it it is dual to M-theory on $\mathrm{AdS}_{3} \times \mathrm{S}^{2} \times \mathrm{CY}$. The central charge of this theory is $c \sim \int_{X} p \wedge p \wedge p$, the dimension of the moduli space of the divisor. If the M5-brane wraps a cycle $\mathcal{M}$ whose size is small relative to the (M-theory Planck) volume of the CY, $V_{X}$, its dynamics can be captured by a field theory which, moreover, can be reduced to a $1+1 \mathrm{~d} \sigma$-model in the limit $R^{6} \gg V_{X}$. The latter is always satisfied in the near-horizon $\mathrm{AdS}_{3}$ decoupling limit so the dual is an effective $\mathrm{CFT}_{2}$ with at least two parameters, $c$ and $V_{X} \cdot V_{X}$ is in a supergravity hypermultiplet and seems to play the role of a coupling constant in the $\sigma$-model. This is also consistent with the validity of 5-d supergravity only in the regime $c \gg V_{X}$.

As emphasized in [27] the M5 under consideration can be thought of as a single very large, smooth brane which does not intersect itself so we may restrict attention to the abelian M5 brane theory living on $\mathcal{M}$. Thus, unlike the previous examples, there does not seem to be any large symmetry group truncating the spectrum at low conformal dimension. Rather, at a generic point, the theory seems to be nothing more than a $\sigma$-model on a parametrically high-dimensional target space (a complex torus bundle over $\mathcal{C P}^{\frac{c}{6}}[52]$ ). Thus at weak coupling one might imagine a degeneracy of order $c$ in the low-lying states of the NS sector (from the $c$ approximately free oscillator in the $\sigma$-model) but this is not consistent with the known spectrum of gravitons in the dual (global) $\mathrm{AdS}_{3}$ theory [53]. This problem even plagues the supersymmetric spectrum as half-BPS states correspond to arbitrary excitations on the non-supersymmetric side of the theory.

It may be the case that some unknown symmetry ${ }^{22}$ truncates the spectrum as occurred in other examples but, lacking this, we will consider alternative explanations here. ${ }^{23}$ The regime of validity of supergravity corresponds to the CY being small on the scale set by the divisor implying a strongly coupled theory. We can thus expect large corrections to the dimensions of generic operators in the theory. It is not surprising then that the large degeneracy of states expected by associating $c$ with the number of degrees of freedom ${ }^{24}$ is not realized at low conformal dimension as the strong-coupling of the theory generically corrects the conformal dimensions of operators. While BPS states are expected to be protected from such corrections the amount of supersymmetry present is not enough to prevent long multiplets from forming from shorter ones and indeed this is observed in orbifold conformal field theories dual to $\mathrm{AdS}_{3} \times \mathrm{S}^{3}$. Unfortunately, as we discuss in section 2.4, there are reasons why this sort of truncation of the spectrum is not entirely satisfactory.

An alternative explanation may be provided by the existence of winding modes along the torus factors in the target space. Turning on three-form flux on the original $M 5$ wrapping the divisor freezes most of its deformation moduli resulting in a much lower dimensional target space. Such configurations, however, necessarily carry $M 2$ charge so this argument would not apply to the sectors of the theory without such charges.

Another possibility is simply that the $\sigma$-model description of the CFT captures only its grossest features (i.e. symmetries and central charges) but is otherwise too naive. While

\footnotetext{
${ }^{22}$ As mentioned in [52] this theory suffers monodromies around singular points in the divisor moduli space and these may constrain the spectrum.

${ }^{23}$ We would like to thank J. de Boer for discussions on several of the possibilities mentioned below.

${ }^{24}$ Recall that in $2-\mathrm{d} c$ is directly related to the local entropy density via Cardy's argument.
} 
this model was used successfully in [27] to reproduce the (subleading) black hole entropy this actually used very few detailed properties of the theory. Thus it is possible that the naive $\sigma$-model description of this theory is incorrect. For instance, the divisor moduli space (the CFT target space) is known to have singular loci where the five-brane self-intersects, generating new light degrees of freedom which may furnish the missing gauge symmetry. One would still have to explain what error is being made in the $\sigma$-model description away from the singular locus.

While we do not understand how the low-energy spectrum of the MSW theory is truncated to match the spectrum of $\mathrm{AdS}_{3}$ we can observe that even in a trivial free-field realization this theory seems to satisfy Cardy's formula already at a $h \sim c$ [54] (whereas the standard Cardy regime is $h \gg c$ with $h$ the conformal dimension). On the other hand without a known mechanism to excise the $c$-dependence of the entropy for $h \ll c$ this will not imply any actual phase transition.

Other examples. It is believed that the theory on $N$ coincident M5 branes in eleven dimensional M-theory flows in the IR to an isolated six dimensional superconformal field theory. The central charge of this CFT scales like $N^{3}$. In the large $N$ limit the CFT is holographically dual to M-theory on $\mathrm{AdS}_{4} \times \mathrm{S}^{7}$. Unfortunately the field theory side of this duality is not well understood but from the bulk it is clear that the boundary CFT should satisfy the properties i), ii), iii) that we have mentioned.

Another class of examples are direct products ${ }^{25}$ of CFTs, each of which has a holographic description, and their small deformations. Examples of this kind were studied in [55-59] and it was argued that in a sense they correspond to multiple emergent AdS throats. These results are consistent with our discussion, as should become evident in later sections. We focus on the case where there is only one copy of AdS space in order to simplify the analysis.

Recently a new interesting class of large $N$ WZW and coset 2d CFTs and their holographic description was considered in [60], also based on earlier work [61-64] where aspects of the large $N$ limit of these CFTs was considered. It would be interesting to explore how they fit into our general discussion.

\subsection{Counter-examples}

Let us consider theories that do not exhibit the properties i), ii), iii) high-lighted above, that we claim are necessary conditions for the existence of a gravitational dual.

CFTs with small central charge. In this paper we are interested in theories with weakly coupled holographic duals; that is with duals where the Planck length is parametrically smaller than the radius of the AdS space. This can only happen when the central charge of the CFT is parametrically large. The reason that we restrict ourselves to this class is that since we have no independent definition of quantum gravity in an AdS space of Planck size we see no way of addressing the question of which CFTs have holographic

\footnotetext{
${ }^{25}$ Here we assume that the number of factors in the product is small compared to the central charge of each of the factors.
} 
duals of this kind. Rather we adopt the perspective that the bulk provides an "effective" description of large $c$ CFTs irrespective of whether it itself is (non-perturbatively) well-defined.

Of course according to the strong version of the AdS/CFT correspondence even the $\mathcal{N}=4$ SYM with small gauge group, for example $\mathrm{SU}(2)$, should have some kind of highly quantum AdS string theory dual, which should be, presumably, independently definable. We do not have any specific reason to doubt this, so CFTs with small central charge are not really counter-examples, but they simply do not fall into the class of weakly coupled holographic duals that we decided to consider in this paper.

Direct products of small CFTs. Another counterexample is to take a CFT with small central charge and construct the direct product ${ }^{26}$ of a large number $N$ of copies of it. Such a CFT has large central charge but the low-lying spectrum differs quite sharply from what we expect in an AdS dual. The number of operators of low conformal dimension grows with $N$, which would indicate that the number of light fields in the dual AdS space would blow up as we take the weak coupling limit, i.e. $\hbar \rightarrow 0$. It is doubtful that such a dual theory would be very useful. Moreover there is no sense in which such a theory factorizes as $N \rightarrow \infty$. Nor is it likely that a small perturbation of this theory will have the correct properties. ${ }^{27}$

Cyclic orbifolds in two dimensions. ${ }^{28}$ Starting with any two dimensional conformal field theory $\mathrm{CFT}_{a}$ we can construct the cyclic orbifold $\left(\mathrm{CFT}_{a}\right)^{N} / Z_{N}$. Like the symmetric orbifold $\left(\mathrm{CFT}_{a}\right)^{N} / S_{N}$, the cyclic orbifold has central charge which is $N$ times that of $\mathrm{CFT}_{a}$ so it satisfies condition i). However its spectrum at low conformal dimension does not satisfy the condition ii): the low-lying spectrum is not $N$-independent, contrary to what happens in symmetric orbifolds. Moreover these theories do not satisfy the factorization condition iii) i.e. they do not have a good $1 / N$ expansion. Hence it seems unlikely that such CFTs have reasonable holographic duals.

\subsection{Do we need gauge-symmetry?}

The collection of examples and counter-examples above leads to a broad picture of which structures are necessary in order for a bulk dual to emerge but it remains to be determined exactly which features are essentially related to holography and which are specific to certain models or classes of models.

In particular what is clearly necessary, as mentioned in the introduction, is:

i) A family of CFTs with $c \rightarrow \infty$ with a bulk dual emerging as a perturbative description about the $c=\infty$ point.

ii) These CFTs contain operators $\left\{\mathcal{O}_{\Delta}\right\}$ whose conformal dimension is $c$-independent, $\Delta \sim \mathcal{O}\left(c^{0}\right)$, and whose degeneracy is also independent of $c$ (while we'll see that conformal invariance imposes a $c$-dependence on the spectrum of operators with $\Delta \sim \mathcal{O}(c)$ ).

\footnotetext{
${ }^{26}$ I.e. without imposing any orbifold symmetrization.

${ }^{27}$ Notice that these problems do not arise if we consider the product of a small number of large CFTs as discussed at the end of the previous subsection.

${ }^{28}$ This counterexample was suggested to us by J. de Boer.
} 
iii) Correlators of the low-lying operators $\left\{\mathcal{O}_{\Delta}\right\}$ above should factorize in the $c \rightarrow$ $\infty$ limit.

While we may impose these as necessary conditions for an abstract CFT to have a bulk dual (and we will try to argue in the following that these requirements are also sufficient) we have not addressed the question of what theories exhibit these properties or, put another way, what underlying structure results in CFTs satisfying i), ii), iii). Clearly the examples above demonstrate that certain theories with a large gauge symmetry fall into the class covered by i), ii), iii) but one of the hopes of this work is to generalize beyond this canonical example.

Unfortunately, aside from abstract conformal field theories, ${ }^{29}$ it is rather difficult to define theories away from weakly coupled points. Let us then consider the following. Suppose that our putative dual CFT has a marginal deformation that takes it to a weakly coupled point. At this point must the CFT be a gauge theory? ${ }^{30}$

An argument in favor of this would be the following. The spectrum of a free theory with central charge $c$ will in general have a $c$-dependent degeneracy ${ }^{31}$ at all conformal dimensions (including the lowest levels). To avoid this we need to manually truncate the spectrum by decoupling a large number of states. This type of decoupling is characteristic of a gauge symmetry which implies that parts of the spectrum are unphysical. We might try to imagine different mechanisms that achieve this truncation but we know of no other candidate in a free theory.

In a strongly coupled theory, on the other hand, it is quite possible that generic operators are massive with a mass scale set by the coupling $\lambda$. Thus for theories without a weakly coupled point the problem cannot be made as sharp but one might still imagine that at $\Delta \sim \lambda$ the $c$-dependence of the spectrum should re-emerge (though such naive arguments may not hold in a strongly coupled theory). Since $\lambda$ is unrelated to $c$ this does not reflect the general structure we expect in the bulk in which the spectrum remains independent of $c$ for all $\Delta \ll c$ in the $c \rightarrow \infty$ limit.

It would be interesting to explore this question further by considering examples such as the MSW CFT where gauge-invariance is certainly not evident but in the following we will sidestep this issue by simply assuming the correct form of the spectrum without concerning ourselves with the mechanism that implements this.

\section{Consistency requirements for a CFT}

Before we proceed we review some basic consistency requirements that have to be satisfied by any unitary conformal field theory.

\footnotetext{
${ }^{29}$ The data for which can be specified in terms of OPE coefficients and conformal dimensions of operators as will be described in the next section.

${ }^{30} \mathrm{By}$ gauge theory we mean also more general examples like symmetric orbifolds that have a large gauge symmetry.

${ }^{31}$ This follows simply from the dependence of both $c\left(C_{T}\right.$ in [65]) and the free energy on the number of free scalar, vector and tensor fields [65].
} 


\subsection{Conformal bootstrap}

Let us say we are given a candidate set of correlation functions for all local operators of a QFT. What are the conditions that they have to satisfy if they are to come from a welldefined conformal field theory? The basic ingredients of a conformal field theory are the conformal primary operators $\mathcal{O}_{i}$, which are labeled by their conformal dimension $\Delta_{i}$ and spin. The normalization of the operators is arbitrary and we can choose a basis so that their 2-point functions, in the case of scalar operators, have the form

$$
\left\langle\mathcal{O}_{i}(x) \mathcal{O}_{j}(y)\right\rangle=\frac{\delta_{i j}}{|x-y|^{2 \Delta_{i}}}
$$

A basic property of a CFT is that there is an operator product expansion (OPE): the product of two local operators can be expressed as a sum over other local operators which has a finite radius of convergence. In general

$$
\mathcal{O}_{i}(x) \mathcal{O}_{j}(y)=\sum_{k} c_{i j}^{k}(x-y) \mathcal{B}_{k}(y)
$$

where the sum runs over all local operators $\mathcal{B}_{k}$, not necessarily primary, and $c_{i j}^{k}(x-y)$ are functions dependent on the dimensions and spins of the operators involved, as well as on the dynamics of the theory. The equality (3.1) holds in the sense that we can replace the product on the l.h.s. by the sum on the r.h.s. inside correlation functions, as long as there are no other operators at smaller distances from $y$ than $|x-y|$.

The expression (3.1) can be greatly simplified by imposing the requirement of conformal invariance. Then it can be shown that the coefficients $c_{i j}^{k}(x-y)$ for the descendant operators can be uniquely determined by kinematics from those between conformal primaries. So all the dynamical information is contained in the OPE coefficients between primaries ${ }^{32}$

$$
\mathcal{O}_{i}(x) \mathcal{O}_{j}(0)=\sum_{k} C_{i j}^{k} \mathcal{O}_{k} \frac{1}{|x|^{\Delta_{i}+\Delta_{j}-\Delta_{k}}}+\text { descendants }
$$

where now $C_{i j}^{k}$ are position independent constants. The full OPE (including the descendant contributions) can be reconstructed as

$$
\mathcal{O}_{i}(x) \mathcal{O}_{j}(y)=\sum_{k} C_{i j}^{k} \hat{\mathcal{F}}_{k}\left(x-y, \partial_{y}\right) \mathcal{O}_{k}(y)
$$

where the differential operator $\hat{\mathcal{F}}_{k}\left(x-y, \partial_{y}\right)$ only depends on kinematics i.e. on the dimensions and spins of the primaries $\mathcal{O}_{i}, \mathcal{O}_{j}, \mathcal{O}_{k}$ and not on the dynamics of the CFT. ${ }^{33}$

The OPE can be used to compute higher $n$-point functions of local operators. Starting with an $n$-point function, we can first take two of the operators which are close to each

\footnotetext{
${ }^{32}$ For simplicity we write down only the contribution from scalar primaries. For intermediate primaries with nonzero spin the form of the OPE is more complicated but still fixed in terms of a single constant.

${ }^{33}$ This differential operator can be determined by multiplying both sides of (3.3) with $\mathcal{O}_{k}(z)$ and demanding that the resulting 3-point function on the l.h.s. is reproduced by the differential operator acting on the 2-point function on the r.h.s., see for example [66].
} 
other and replace them with their complete OPE. In this way we can rewrite the $n$-point function as an (infinite) sum over $(n-1)$-point functions. Similarly the $(n-1)$-point functions can be reduced by an OPE to sums over $(n-2)$-point functions and so on, until we get to the basic 2- and 3-point functions. Hence the entire CFT can be reconstructed from knowing the spectrum of conformal primaries $\mathcal{O}_{i}$, i.e. their conformal dimensions $\Delta_{i}$ and spins, and the 3-point functions $C_{i j}^{k}$.

A natural question is whether every choice of the data $\left(\Delta_{i}, C_{i j}^{k}\right)$ corresponds to a consistent conformal field theory. The answer is negative and this can be understood as follows. When we use successive OPEs to reduce the $n$-point functions to 2- and 3-point functions there is an ambiguity in the choice of the order that we perform the OPE. To end up with a consistent theory the answer for the $n$-point function should be independent of the order in which the OPEs are performed. This leads to certain conditions that have to be satisfied by the data $\left(\Delta_{i}, C_{i j}^{k}\right)$.

This can be seen in the simplest way by considering a four point function

$$
\left\langle\mathcal{O}_{1}\left(x_{1}\right) \mathcal{O}_{2}\left(x_{2}\right) \mathcal{O}_{3}\left(x_{3}\right) \mathcal{O}_{4}\left(x_{4}\right)\right\rangle
$$

which can evaluated by doing the OPE between the two operators at $x_{1}$ and $x_{2}$ and at the same time between $x_{3}$ and $x_{4}$, or by doing the OPE between $x_{1}$ and $x_{4}$ and at the same time between $x_{2}$ and $x_{3}{ }^{34}$. In the first case, i.e. in the channel $(12) \rightarrow(34)$, the 4-point function becomes

$$
\left\langle\mathcal{O}_{1}\left(x_{1}\right) \mathcal{O}_{2}\left(x_{2}\right) \mathcal{O}_{3}\left(x_{3}\right) \mathcal{O}_{4}\left(x_{4}\right)\right\rangle=\sum_{k} C_{12}^{k} C_{34}^{k} \hat{\mathcal{F}}_{k}\left(x_{1}-x_{2}, \partial_{x_{2}}\right) \hat{\mathcal{F}}_{k}\left(x_{3}-x_{4}, \partial_{x_{4}}\right) \frac{1}{\left|x_{2}-x_{4}\right|^{2 \Delta_{k}}}
$$

As we explained, the only terms in this expression which are dependent on the dynamics are the OPE coefficients $C_{12}^{k}, C_{34}^{k}$. So we introduce the following functions

$$
\mathbf{G}_{k}^{12,34}\left(x_{1}, x_{2}, x_{3}, x_{4}\right) \equiv \hat{\mathcal{F}}_{k}\left(x_{1}-x_{2}, \partial_{x_{2}}\right) \hat{\mathcal{F}}_{k}\left(x_{3}-x_{4}, \partial_{x_{4}}\right) \frac{1}{\left|x_{2}-x_{4}\right|^{2 \Delta_{k}}}
$$

The functions $\mathbf{G}_{k}^{12,34}$ are the so-called conformal blocks, or conformal partial waves (CPWs). They correspond to the contribution of an operator $\mathcal{O}_{k}$ and all of its descendants to the double OPE between (12) and (34). The conformal blocks are determined by kinematics of the conformal group. Explicit expressions for the CPWs in the case of $d=4$ can be found in $[66,67]$ and are summarized in appendix A. Once the kinematic factors have been absorbed into the CPWs, all of the dynamics of the theory lies in the information about conformal dimensions and the 3-point functions $C_{i j}^{k}$.

We evaluated the 4-point function by performing the double OPE in the "direct channel" $(12) \rightarrow(34)$. Alternatively we could have performed the OPE in the "crossed channel" $(14) \rightarrow(23)$ and we would have derived a similar expression as (3.4) with the roles of the points 2 and 4 interchanged. Demanding that the resulting 4-point function is the same

\footnotetext{
${ }^{34}$ There is also the channel $(13) \rightarrow(24)$ but it is sufficient to check the conformal bootstrap between the other two channels mentioned above.
} 


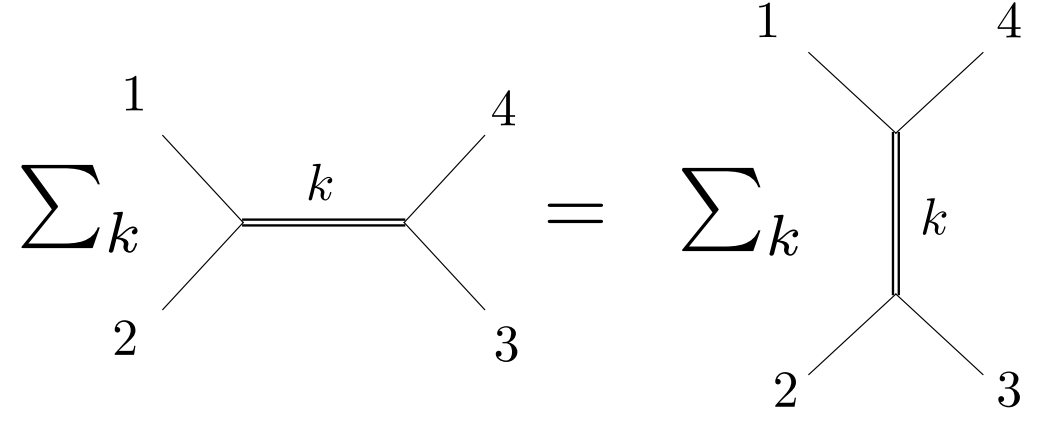

Figure 2. Conformal bootstrap condition for the 4-point function.

gives the following condition

$$
\sum_{k} C_{12}^{k} C_{34}^{k} \mathbf{G}_{k}^{12,34}\left(x_{1}, x_{2}, x_{3}, x_{4}\right)=\sum_{k} C_{14}^{k} C_{23}^{k} \mathbf{G}_{k}^{14,23}\left(x_{1}, x_{4}, x_{2}, x_{3}\right)
$$

which is called the conformal bootstrap condition (also called "crossing symmetry", if the four external operators are the same) and is depicted schematically in figure 1. Notice that we have such a condition for each choice of the four external operators. Any consistent CFT must satisfy the bootstrap condition. ${ }^{35}$ It can be shown that if the bootstrap condition is satisfied for the 4-point function then the reduction of all higher $n$-point functions via successive OPEs will be consistent, independent of the order that we choose to perform the OPE.

Notice that the unitarity of the theory requires that the coefficients $C_{i j k}$ are real. See [68-73] for recent work were the unitarity of the CFT in $d>2$ was used to derive certain constraints for the data $\Delta_{i}, C_{i j k}$, in special cases.

\subsection{Modular invariance and consistency at finite temperature}

The conditions (3.6) are necessary conditions for the consistency of the CFT. However they are not sufficient. This can be seen even in the simpler case of two dimensional CFTs. While the conformal bootstrap equations guarantee the consistency of correlators on the plane, the same is not true if we place the theory on a nontrivial manifold, for example the torus or the cylinder. ${ }^{36}$ Then either by modular invariance or by arguments similar to those described below we find new constraints on the theory which go beyond the conformal bootstrap conditions.

In two dimensions modular invariance is derived by considering the CFT on a torus. Conformal invariance together with invariance under large diffeomorphisms of the torus

\footnotetext{
${ }^{35}$ Let us notice that if we have $N$ conformal primaries, then the number of data $\left(\Delta_{i}, C_{i j}^{k}\right)$ grows like $N^{3}+N$. On the other hand the number of conditions of the form (3.6) grows like $N^{4}$. This suggests that it might be possible to fully determine the dynamics of all CFTs simply by imposing the bootstrap conditions. In practice it is difficult to solve these conditions, especially since $N$ is infinite. The program of solving CFTs via the "conformal bootstrap" approach has had some success in special two dimensional CFTs (for example in the case of the minimal models).

${ }^{36}$ The requirement of having a consistent theory on certain nontrivial manifolds (i.e. $\mathbf{R}^{d-1} \times \mathbf{S}^{1}$ or $\mathbf{S}^{d-1} \times$ $\mathbf{S}^{1}$ ) corresponds, from a physical point of view, to demanding consistency of the CFT at finite temperature.
} 
implies that the path integral of the theory must be invariant under the transformation $\tau \rightarrow-1 / \tau$ of the Teichmuller parameter. This implies an invariance of the thermal partition function under the transformation $T \rightarrow 1 /\left(L^{2} T\right)$ of the temperature, where $L$ is the length of the spatial circle on which the CFT is defined. This relates the spectrum of large conformal dimension operators (which dominate at large $T$ ) to that of low lying spectrum (which dominate for small $T$ ).

Modular invariance is a very strong constraint on the spectrum of a CFT and is moreover independent of the conformal bootstrap conditions on the plane. To see this let us consider a two dimensional CFT consisting only of the Virasoro module of the identity operator (which includes the stress tensor $T(z)$ ). All $n$-point functions of this theory, for example correlators of the form $\left\langle T\left(z_{1}\right) \ldots T\left(z_{n}\right)\right\rangle$ can be directly computed because of holomorphy ${ }^{37}$ and they only depend on the central charge $c$. It is straightforward to show that they satisfy the bootstrap equations if we assume that fields only fuse within the $T$-module. So the theory seems to be consistent on the plane, in the sense that crossing symmetry is satisfied. On the other hand its partition function is not modular invariant, ${ }^{38}$ hence such a theory cannot exist.

In higher dimensions there is no direct analogue of modular invariance. If we consider the CFT on a $d$-dimensional torus $\mathbf{T}^{d}$ then the partition function has to be invariant under $\operatorname{SL}(d, \mathbf{Z})$ large diffeomorphisms but it is not clear how to translate this invariance to a condition for the spectrum or correlators of the CFT. ${ }^{39}$ Nonetheless demanding the consistency of the CFT at finite temperature does indeed introduce some new constraints which go beyond the bootstrap conditions. We refer to such constraints as "generalized modular invariance" though they are clearly much more difficult to analyze than modular invariance of a two dimensional CFT on a torus.

To begin, let us consider a conformal field theory on $\mathbf{R}^{d-1} \times \mathbf{S}^{1}$ where the size of $\mathbf{S}^{1}$ is $\beta=\frac{1}{T}$. Notice that on this space, unlike what happens on $\mathbf{R}^{d}$, operators can have nonzero expectation values: we have introduced the scale $\beta$, so an operator $\mathcal{O}_{k}$ of dimension $\Delta_{k}$ can have an expectation value of the form

$$
\left\langle\mathcal{O}_{k}\right\rangle_{\beta}=\frac{A_{k}}{\beta^{\Delta_{k}}}
$$

where the constant $A_{k}$ is independent of $\beta$.

These thermal 1-point functions can be computed from the 3-point functions $C_{i j}^{k}$ as follows: consider the CFT living on a sphere $\mathbf{S}^{d-1}$ of radius 1 , at inverse temperature $\beta$.

\footnotetext{
${ }^{37}$ An $n$-point function of this form is meromorphic with respect to any of its arguments, so it can be determined by its singularities. It has poles whenever one of the operators approaches the other insertions. These poles are fixed by the TT OPE which is completely determined by conformal invariance and only contains $T$ and its descendants. Hence the poles of this correlator are related to $(n-1)$-point functions of $T$. Recursively all $n$-point functions of this type can be reconstructed from the TT 2-point function and the TT OPE. The answer depends only on the value of the central charge $c$.

${ }^{38}$ Defining $q=e^{-\beta}$ the partition function of this theory would be $Z(q)=q^{-c / 24} \prod_{n=2}^{\infty} \frac{1}{1-q^{n}}$ which is not modular invariant, so it cannot be a consistent theory.

${ }^{39}$ We would like to thank J. de Boer for discussions related to this.
} 
By definition we have

$$
\left\langle\mathcal{O}_{k}\right\rangle_{\beta}^{\prime}=\frac{\sum_{|\psi\rangle}\left\langle\psi\left|\mathcal{O}_{k}\right| \psi\right\rangle e^{-\beta E_{\psi}}}{Z(\beta)}
$$

where $Z(\beta) \equiv \sum_{|\psi\rangle} e^{-\beta E_{\psi}}$ is the thermal partition function of the system. The sum runs over all states of the Hilbert space, $E_{\psi}$ is the energy of the state $|\psi\rangle$ and the prime refers to the fact that the 1-point function is evaluated on $\mathbf{S}^{d-1} \times \mathbf{S}^{1}$ unlike (3.7) which was on $\mathbf{R}^{d-1} \times \mathbf{S}^{1}$. Using the state operator map $|\psi\rangle \leftrightarrow \mathcal{O}$ this can be thought of as a sum over all local operators $\mathcal{O}$ of the CFT (not necessarily conformal primaries) with the identifications $E_{\psi} \leftrightarrow \Delta_{\mathcal{O}}$ and $\left\langle\psi\left|\mathcal{O}_{k}\right| \psi\right\rangle \leftrightarrow C_{\mathcal{O O O O}_{k}}$ (assuming we work in a basis where the $\mathcal{O}$ 's are hermitian). The high temperature limit of the 1-point function on $\mathbf{S}^{d-1} \times \mathbf{S}^{1}$ should be the same as that on $\mathbf{R}^{d-1} \times \mathbf{S}^{1}$, in other words $\lim _{\beta \rightarrow 0} \frac{\left\langle\mathcal{O}_{k}\right\rangle^{\prime}}{\left\langle\mathcal{O}_{k}\right\rangle}=1$, so we finally find

$$
A_{k}=\lim _{\beta \rightarrow 0}\left(\beta^{\Delta_{k}} \frac{\sum_{\mathcal{O}} C_{\mathcal{O O O}_{k}} e^{-\beta \Delta_{\mathcal{O}}}}{\sum_{\mathcal{O}} e^{-\beta \Delta_{\mathcal{O}}}}\right)
$$

Thus in principle if we know all the data $\left(\Delta_{k}, C_{i j}^{k}\right)$ of the zero temperature CFT, we can compute the thermal 1-point functions $A_{k}$.

Let us now consider correlation functions of operators at finite temperature starting with a thermal 2-point function $\left\langle\mathcal{O}_{i}(x) \mathcal{O}_{j}(y)\right\rangle_{\beta}$. If the points $x$ and $y$ are close to each other then we can consider the OPE between the two operators. Notice that the OPE is a short distance expansion (or in other words an operator statement) so it should hold even when the theory is placed at finite temperature. The only difference is that operators on the r.h.s. can have nontrivial 1-point functions. In other words, if we know the exact OPE of two operators

$$
\mathcal{O}_{i}(x) \mathcal{O}_{j}(y)=\sum_{k} C_{i j}^{k}(x-y) \mathcal{O}_{k}(y)
$$

then we can express the thermal 2-point function of the operators in terms of 1-point functions as

$$
\left\langle\mathcal{O}_{i}(x) \mathcal{O}_{j}(y)\right\rangle_{\beta}=\sum_{k} C_{i j}^{k}(x-y)\left\langle\mathcal{O}_{k}\right\rangle_{\beta}=\sum_{k} C_{i j}^{k}(x-y) \frac{A_{k}}{\beta^{\Delta_{k}}}
$$

where the constants $A_{k}$ were introduced in (3.7). Notice that this has to be understood as an expansion for $x \rightarrow y$, which may not converge when $|x-y|>\beta$, but which can (presumably) be analytically continued everywhere. ${ }^{40}$ Of course the analytic continuation can be done only after summing over $k$ and not term-by-term, so in practice it may be difficult to perform.

The 2-point function constructed in this way has to be periodic around the thermal circle (i.e. to satisfy the KMS condition)

$$
\left\langle\mathcal{O}_{i}(\tau, \mathbf{x}) \mathcal{O}_{j}(y)\right\rangle_{\beta}=\left\langle\mathcal{O}_{i}(\tau+\beta, \mathbf{x}) \mathcal{O}_{j}(y)\right\rangle_{\beta}
$$

where $\mathbf{x}$ is the coordinate on $\mathbf{R}^{d-1}$. This already imposes some new conditions among the 3 -point functions $C_{i j}^{k}$ and the conformal dimensions $\Delta_{i}$ via equations (3.8), (3.9) and (3.10).

\footnotetext{
${ }^{40}$ See $[74,75]$ for some applications of the finite temperature OPE in $d>2$ dimensional CFTs.
} 

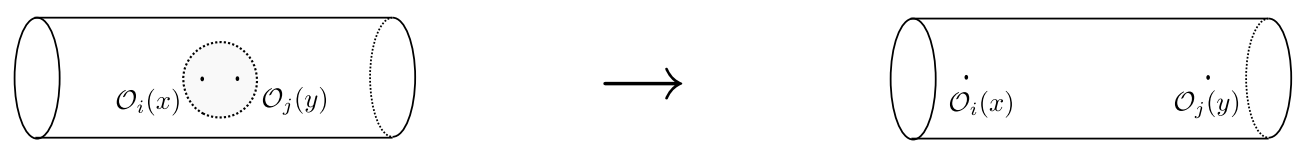

Figure 3. The thermal 2-point function $\left\langle\mathcal{O}_{i}(x) \mathcal{O}_{j}(y)\right\rangle_{\beta}$ can be evaluated by the OPE in the limit $x \rightarrow y$, in terms of thermal 1-point functions. In the opposite limit where $|x-y| \rightarrow \infty$ it should factorize to the product of 1-point functions $\left\langle\mathcal{O}_{i}\right\rangle_{\beta}\left\langle\mathcal{O}_{j}\right\rangle_{\beta}$.

Moreover when we consider the limit where $|x-y| \rightarrow \infty$, i.e. when the two points are widely separated in the spatial directions, we expect that the 2-point function will factorize to a product of 1-point functions

$$
\lim _{|x-y| \rightarrow \infty}\left\langle\mathcal{O}_{i}(x) \mathcal{O}_{j}(y)\right\rangle_{\beta}=\left\langle\mathcal{O}_{i}\right\rangle_{\beta}\left\langle\mathcal{O}_{j}\right\rangle_{\beta}=\frac{A_{i} A_{j}}{\beta^{\Delta_{i}+\Delta_{j}}}
$$

This condition gives more non-trivial constrains for the data $\left(\Delta_{i}, C_{i j}^{k}\right) .{ }^{41}$

Finally we have the following condition: let us consider a small variation of the temperature. The temperature is related to the size of the thermal circle, or equivalently to the value of the metric component $g_{00}$. Changing the temperature can be understood as changing $g_{00}$. We know that by its definition, the operator $T_{00}$ is "dual" to the component $g_{00}$ in the sense that $g_{00}$ is the source for $T_{00}$. Changing the metric infinitesimally is equivalent to inserting the stress tensor in the correlators. Hence we arrive at the following relation

$$
\frac{\partial\left\langle\mathcal{O}_{k}\right\rangle_{\beta}}{\partial \beta}=-\frac{1}{\beta} \int d^{d} x\left\langle T_{00}(x) \mathcal{O}_{k}(0)\right\rangle_{\beta}^{c}
$$

where the integral ${ }^{42}$ is over $\mathbf{R}^{d-1} \times \mathbf{S}^{1}$ and the superscript $c$ in the 2-point function stands for "connected". ${ }^{43}$ The l.h.s. of this equation can be immediately evaluated from (3.7) while the r.h.s. from the (analytic continuation of) the OPE (3.9). The set of equations (3.12) (one equation for each operator $\mathcal{O}_{k}$ ) give additional nontrivial conditions that have to be satisfied by any conformal field theory in any dimension.

To summarize, in this section we have formulated some additional general constraints (3.10), (3.11) and (3.12) for consistent CFTs which go beyond the conformal bootstrap conditions, though we have not analyzed these constraints sufficiently to understand the extent to which they are all independent. As we will see in the next subsection they impose some non-trivial conditions on the spectrum of operators, reminiscent of those coming from modular invariance in two dimensional CFTs.

Before we close this section let us mention that the constraints from modular invariance of the boundary CFT, in the context of the $\mathrm{AdS}_{3} / \mathrm{CFT}_{2}$ correspondence, have been recently discussed in [76-81].

\footnotetext{
${ }^{41}$ The constants $A_{k}$ can be computed from (3.8) and then the l.h.s. by the (analytic continuation of) (3.9)).

${ }^{42}$ The integral has to be regularized and (temperature-independent) divergences have to be removed.

${ }^{43}$ The connected 2-point function of two operators is defined as $\left\langle\mathcal{O}_{i}(x) \mathcal{O}_{j}(y)\right\rangle_{\beta}^{c} \equiv\left\langle\mathcal{O}_{i}(x) \mathcal{O}_{j}(y)\right\rangle_{\beta}-$ $\left\langle\mathcal{O}_{i}(x)\right\rangle_{\beta}\left\langle\mathcal{O}_{j}(y)\right\rangle_{\beta}$.
} 


\section{3 "Cardy formula" and higher dimensional CFTs}

Notice that in a two-dimensional CFT if we apply the condition (3.12) by taking the operator $\mathcal{O}_{k}$ to be $T_{00}$ itself, then we can derive the Cardy formula without direct reference to modular invariance: on general grounds the thermal expectation value of the stress tensor on $\mathbf{R} \times \mathbf{S}^{1}$ (i.e. the thermal energy density) has the form

$$
\left\langle T_{00}\right\rangle_{\beta}=\frac{\pi}{6} \widetilde{c} T^{2}
$$

where $T$ is the temperature. The constant $\widetilde{c}$ is a priori unrelated to the central charge $c$, defined by the 2-point function of the stress-energy tensor on the plane. Standard thermodynamic arguments imply that the associated entropy density is

$$
s=\frac{\pi}{3} \widetilde{c} T
$$

Now, the thermal 2-point function $\left\langle T_{00}(x) T_{00}(y)\right\rangle_{\beta}$ in a $2 \mathrm{~d}$ CFT can be exactly computed by the cylinder-to-plane exponential map and is proportional to the central charge c. By imposing condition (3.12) and performing the integral one can then show [82] that in two dimensions

$$
\widetilde{c}=c
$$

This leads to the Cardy formula, which determines the entropy of the CFT at high temperatures in terms of the central charge $c$.

Can this argument be used in higher dimensional CFTs to relate the thermal entropy density to the "central charge" of the CFT? In a higher dimensional CFT the entropy density has the form $s \sim \widetilde{c} T^{d-1}$. Let us call $c$ the constant which appears in the 2point function of the stress tensor in flat space. While equation (3.12) is still true for higher dimensional CFTs, the argument described above cannot be applied and there is no analogue of the Cardy formula. In particular, the constants $c$ and $\widetilde{c}$ can in general be different (and do differ in known examples ${ }^{44}$ ). The problem with applying the previous argument is that there is no way to evaluate the thermal 2-point function $\left\langle T_{00}(x) T_{00}(y)\right\rangle_{\beta}$ in a simple way, unlike what happens in $2 \mathrm{~d}$ CFTs. In higher dimensions there is no analogue of the exponential map between $\mathbf{R}^{d-1} \times \mathbf{S}^{1}$ and $\mathbf{R}^{d}$ and the thermal 2-point function of the stress tensor is not fixed by conformal invariance. However, as we discuss in section 7.1, we may still be able to use the condition (3.12) to derive some qualitative statements about the high-temperature entropy of the CFT, in the spirit of the Cardy formula.

\section{Generalized Free CFTs and holography}

We now return to our goal of identifying the class of $d$-dimensional CFTs for which a holographic gravitational theory provides an effective description, at least in some regime.

As we explained in the previous sections, the minimum structure necessary in order for a CFT to have a (weakly coupled) bulk dual is that it contains in its Hilbert space

\footnotetext{
${ }^{44}$ For example in the large $N \mathrm{SU}(N) \mathcal{N}=4 \mathrm{SYM}$ the ratio $\widetilde{c} / c$ is believed to continuously interpolate between the value $4 / 3$ at $\lambda \ll 1$ and the value 1 at $\lambda \gg 1$. Also in free four-dimensional CFTs the ratio $\widetilde{c} / c$ can take various values, see [83] for a recent discussion.
} 
a sector which consists of Generalized Free Fields (GFF) i.e. operators whose correlators factorize. By analyzing a CFT containing such fields we are naturally led to the notion of "multiparticle states". These GFF and the multiparticle states are defined in an expansion about large central charge, $c \rightarrow \infty$, and satisfy crossing symmetry to zeroth order in a $1 / c$ expansion. Even at this order, however, we will find an inconsistency emerging from attempting to reconcile the spectrum of this theory with other constraints from conformal invariance: such CFTs fail to satisfy the "generalized modular invariance" conditions mentioned in 3.2 .

For finite but large $c$ we argue that our analysis of the spectrum receives corrections for operators of large dimension (states with $\Delta \sim c$ ) resolving the inconsistency. This hints at an essential property of such theories that is manifest in their dual bulk description: they must be completed by the addition of "black hole states" satisfying $\Delta \sim c$. We will return to this notion in later sections.

\subsection{Generalized free fields: factorization for $\Delta>\frac{d-2}{2}$}

Let us now explain what is special about "generalized free fields". Intuitively we think of a field as being "free" when it obeys linear equations of motion. A basic consequence of linearity is that it allows the superposition of solutions, or superposition of excitations of the field. Similarly, we think of a "weakly interacting" field as one which is a small deformation of a free one, i.e. when it obeys equation of motion which have small nonlinearities around linear equations of motion. The nonlinearities are controlled by a coupling constant $g$, and as $g \rightarrow 0$ the field becomes linear.

In a CFT in $d$ spacetime dimensions, the condition that a scalar operator $\mathcal{O}$ is free is equivalent to the fact that its conformal dimension is $\Delta=\frac{d-2}{2}$. This can be shown from the conformal algebra. To see whether $\nabla^{2} \mathcal{O}=0$ we consider the norm of the state $P_{\mu} P^{\mu}|\mathcal{O}\rangle$. Using the conformal algebra we find that

$$
\| P_{\mu} P^{\mu}|\mathcal{O}\rangle\left\|^{2}=\left\langle\mathcal{O}\left|K_{\nu} K^{\nu} P_{\mu} P^{\mu}\right| \mathcal{O}\right\rangle=8 d \Delta\left(\Delta-\frac{d-2}{2}\right)\right\||\mathcal{O}\rangle \|^{2}
$$

so the condition $\nabla^{2} \mathcal{O}=0$ is equivalent to $\Delta=\frac{d-2}{2}$.

However there is another notion of a free field, characterized by the fact that its correlation functions factorize to products of 2-point functions. For a standard free field with $\Delta=\frac{d-2}{2}$ the factorization is an immediate consequence of the equation of motion $\nabla^{2} \mathcal{O}=0$. However, more generally we may have fields with $\Delta>\frac{d-2}{2}$ whose correlators factorize. These are called "generalized free fields". The reason that such fields should be called free is that there is a sense in which we can superimpose excitations created by these fields, as a result of their factorization property. Generalized free fields have been discussed before, in various contexts, in the literature [17]. However, as we will explain later, our perspective is different since we argue that such fields can only exist in a limiting sense (so some statements in the older literature may be less relevant from our point of view).

More precisely let us define a generalized free field ${ }^{45} \mathcal{O}$ of dimension $\Delta>\frac{d-2}{2}$ as one

\footnotetext{
${ }^{45}$ By the term "field" we mean a local operator in the CFT, not necessarily a fundamental field of the Lagrangian. If the CFT is a gauge theory we are obviously talking about gauge invariant operators.
} 
whose correlators take the form

$$
\left\langle\mathcal{O}\left(x_{1}\right) \ldots \mathcal{O}\left(x_{n}\right)\right\rangle=\left\langle\mathcal{O}\left(x_{1}\right) \mathcal{O}\left(x_{2}\right)\right\rangle \ldots\left\langle\mathcal{O}\left(x_{n-1}\right) \mathcal{O}\left(x_{n}\right)\right\rangle+\text { permutations }
$$

In particular $n$-point functions for $n$ odd vanish ${ }^{46}$. One peculiar property of these fields is that while they are free, in the sense that the obey some kind of superposition principle ${ }^{47}$, they do not obey linear equations of motion. In particular, because $\mathcal{O}$ has the wrong dimension to be a free field we cannot describe it in terms of a local free Lagrangian in the $d$-dimensional flat space where the CFT lives.

For example if we choose the normalization of the operators appropriately then the 2 -point function is

$$
\langle\mathcal{O}(x) \mathcal{O}(y)\rangle=\frac{1}{|x-y|^{2 \Delta}}
$$

which does not obey a linear differential equation, unless $\Delta=\frac{d-2}{2}$. As we will see in section 5 the lack of linear equations for such fields on the boundary is an important point related to their holographic description.

\subsection{Conformal bootstrap for generalized free fields and multiparticle spectrum}

Let us now make the assumption that our CFT contains in its spectrum a GFF i.e. a scalar operator $\mathcal{O}$ of dimension $\Delta>\frac{d-2}{2}$ whose correlators factorize. What conclusions can we draw from this?

It is easy to see that the bootstrap conditions cannot be satisfied if we assume that $\mathcal{O}$ is the only operator in the CFT. In particular we will see that we must introduce composite operators made out of products of $\mathcal{O}$. These are the equivalent of multi-trace operators in gauge theories. Thus the existence of a generalized free field, combined with crossing symmetry automatically implies a Fock space structure for the Hilbert space.

For example let us consider the 4-point function

$$
\left\langle\mathcal{O}\left(x_{1}\right) \mathcal{O}\left(x_{2}\right) \mathcal{O}\left(x_{3}\right) \mathcal{O}\left(x_{4}\right)\right\rangle=\frac{1}{\left|x_{12}\right|^{2 \Delta}\left|x_{34}\right|^{2 \Delta}}+\text { permutations }
$$

By considering a conformal partial wave expansion of the correlator in the channel $(12) \rightarrow(34)$ we can infer that the conformal field theory must contain a tower of conformal primary operators with conformal dimension $2 \Delta+2 n+l$ and $\operatorname{spin} l$ [66]. These operators can be written as $\mathcal{O}_{n, l}^{(2)}=: \mathcal{O} \hat{\partial}_{\left[\mu_{1}\right.} \ldots \hat{\partial}_{\left.\mu_{l}\right]}\left(\hat{\nabla}^{2}\right)^{n} \mathcal{O}:$ where the brackets denote the symmetric traceless combination and $\hat{\partial} \equiv \vec{\partial}-\overleftarrow{\partial}$ in order to project out descendant contributions. Intuitively these operators can be understood as "two-particle" states made out of the field $\mathcal{O}$.

In other words the OPE of $\mathcal{O}$ with itself has the form

$$
\mathcal{O}(x) \mathcal{O}(0)=\frac{1}{|x|^{2 \Delta}}+\sum_{n, l} C_{n, l}\left(|x|^{2 n+l} \mathcal{O}_{n, l}^{(2)}+\text { descendants }\right)
$$

\footnotetext{
${ }^{46}$ Conformal invariance implies that one point functions vanish in a conformally invariant vacuum.

${ }^{47}$ This is due to factorization. For example, the energy of states created by acting repeatedly with the field $\mathcal{O}$ is additive. In gauge theory language this is expressed by the fact that the conformal dimension of a multi-trace operator is equal to the sum of the conformal dimensions of its constituent single-trace operators, at infinite $N$.
} 
with OPE coefficients $C_{n, l}$ computed in [66] and we have suppressed Lorentz indices for simplicity.

Notice that the existence of the operators $\mathcal{O}_{n, l}^{(2)}$ is a very nontrivial statement from the CFT point of view: in a general CFT if we have an operator $\mathcal{O}$ with dimension $\Delta$ there is no reason to expect the existence of an operator : $\mathcal{O O}$ : of dimension $2 \Delta$. It is precisely the fact that our field $\mathcal{O}$ is, in some sense, "free" that is responsible for the existence of such operators. By analogy with large $N$ gauge theories or via a presumed gravitational dual, we will refer to these new operators as multi-particle operators. Likewise the original $\mathcal{O}$ we will refer to as a single-particle operator.

Correlation functions (or OPEs) of the 2-particle operators $\mathcal{O}_{n, l}^{(2)}$ can be computed by taking limits of correlators of $\mathcal{O}$. For example, to compute the 3 -point function of 2-particle operators

$$
\left\langle\mathcal{O}_{n_{1}, l_{1}}^{(2)}\left(x_{1}\right) \mathcal{O}_{n_{2}, l_{2}}^{(2)}\left(x_{2}\right) \mathcal{O}_{n_{3}, l_{3}}^{(2)}\left(x_{3}\right)\right\rangle
$$

we start by considering the 6-point function of the single-particle operator $\mathcal{O}$, which by the assumption of factorization is

$$
\left\langle\mathcal{O}\left(x_{1}\right) \ldots \mathcal{O}\left(x_{6}\right)\right\rangle=\frac{1}{\left|x_{12}\right|^{2 \Delta}\left|x_{34}\right|^{2 \Delta}\left|x_{56}\right|^{2 \Delta}}+\text { permutations }
$$

By acting with the appropriate combination of derivatives, considering the $x_{12}, x_{34}, x_{56} \rightarrow 0$ limit and using the OPE (4.4) for each of the pairs of points (12), (34), (56) we can isolate the desired 3-point function (4.5). Similarly we can also determine the OPE between 2particle operators. Working iteratively this way (making further subtractions before taking the limits) we can determine the OPEs of various two-particle states. Thus the OPEs of the two-particle states are completely determined by the OPE of the single-particle states.

Similarly by considering the conformal partial wave expansion of correlators of 2particle operators with $\mathcal{O}$ we can infer the existence of operators with the quantum numbers corresponding to "3-particle" operators : $\mathcal{O O O}$ : etc. Following this procedure we can inductively show that the conformal field theory must contain a sector of operators which has the structure of a freely generated Fock space, where the basic excitation has the quantum numbers of the operator $\mathcal{O}$ and of its conformal descendants. We want to emphasize that this follows from the single assumption that correlators of the field $\mathcal{O}$ factorize.

More generally we consider a CFT which has more than one generalized free fields, which we call $\mathcal{O}_{i}$. By definition these are operators whose correlators factorize and they define the set of single-particle states in the theory. By considering the conformal partial wave decomposition of various correlators of such operators we can show that the theory will have to contain the "multi-particle" operators which can be constructed out of the main building blocks $\mathcal{O}_{i}$. For example if we have two generalized free fields $\mathcal{O}_{1}, \mathcal{O}_{2}$ of conformal dimension $\Delta_{1}, \Delta_{2}$, then by considering the 4-point function

$$
\left\langle\mathcal{O}_{1}\left(x_{1}\right) \mathcal{O}_{2}\left(x_{2}\right) \mathcal{O}_{1}\left(x_{3}\right) \mathcal{O}_{2}\left(x_{4}\right)\right\rangle=\frac{1}{\left|x_{13}\right|^{2 \Delta_{1}}\left|x_{24}\right|^{2 \Delta_{2}}}
$$

and decomposing it in CPWs in the $(12) \rightarrow(34)$ channel we can see that there must be a scalar operator with dimension $\Delta_{1}+\Delta_{2}$ which we denote by : $\mathcal{O}_{1} \mathcal{O}_{2}$ : i.e. a "two-particle" operator, and so on. 


\subsection{Partition functions of generalized free fields}

While the freely generated Fock space structure described above looks reasonable at first sight, it suffers from some pathologies related to the conditions of "generalized modular invariance" that we reviewed in section 3.2. For simplicity let us assume that there is only one generalized free field $\mathcal{O}$ in the theory. The "single particle states" are operators of the form

$$
\mathcal{O}, \partial_{i} \mathcal{O}, \partial_{i} \partial_{j} \mathcal{O} \ldots
$$

of dimension $\Delta, \Delta+1, \Delta+2 \ldots$. The 2-particle states are

$$
: \mathcal{O O}:,: \mathcal{O} \partial_{i} \mathcal{O}:, \ldots
$$

with dimensions $2 \Delta, 2 \Delta+1, \ldots$

The single particle states form a representation of the conformal group $\mathrm{SO}(2, d)$. Let us call this representation $V_{\Delta}$. The 2-particle states also form a representation, which can be understood as $\operatorname{Sym}\left(V_{\Delta} \otimes V_{\Delta}\right)$, where $S y m$ stands for symmetrization reflecting the fact that the excitations behave like identical bosons. More generally the $N$-particle states are the representation $\operatorname{Sym}\left(\otimes^{N} V_{\Delta}\right)$.

Let us now consider the generalized free CFT on $\mathbf{S}^{d-1} \times$ time and compute its thermal partition function ${ }^{48}$

$$
Z(q)=\sum_{\text {operators }} q^{\Delta}, \quad q=e^{-\beta}
$$

where $\beta=\frac{1}{T}$ is the inverse temperature and we take the radius of the $\mathbf{S}^{d-1}$ to be equal to one (we will restore it in the final formula). In principle we have to sum over one, two-, three- etc. particle states. However since the Hilbert space is a freely generated Fock space, we can use a standard statistical mechanics argument to determine the full partition function, starting from the "single-particle partition function". We first define the single-particle partition function as

$$
Z_{1}(q)=\sum_{\text {single particle }} q^{\Delta}
$$

Then the multi-particle partition function is given by the formula ${ }^{49}$

$$
\log Z(q)=\sum_{n=1}^{\infty} \frac{1}{n} Z_{1}\left(q^{n}\right)
$$

Let us apply this formula to the generalized free CFT, where for simplicity we will assume that there is only one generalized free field $\mathcal{O}$.

\footnotetext{
${ }^{48}$ In this section we ignore contribution from the Casimir energy on the sphere.

${ }^{49}$ The way to understand this formula the following: each of the single particle states behaves as a simple harmonic oscillator which can be excited arbitrary number of times so its partition function is $\frac{1}{1-q^{\Delta}}$. Since all oscillators are independent the full partition function is $Z(q)=\prod_{\Delta} \frac{1}{1-q^{\Delta}}$. Taking the logarithm, using the expansion $\log (1-q)=-\sum_{n=1}^{\infty} \frac{q^{n}}{n}$ and interchanging the order of summation over $\Delta$ and $n$ we arrive at (4.7).
} 
In the case of a generalized free field $\mathcal{O}$ with $\Delta>\frac{d-2}{2}$ all single particle states can be constructed by acting on $\mathcal{O}$ with an arbitrary number of derivatives $\partial_{i}, i=1, \ldots, d$, where $d$ is the spacetime dimension of the CFT. The derivatives are all independent. Adding a derivative increases the conformal dimension by 1 . Since the derivatives are independent we can think of them as $d$ independent harmonic oscillators. Then the single particle partition function is simply

$$
Z_{1}(q)=q^{\Delta} \frac{1}{(1-q)^{d}}
$$

and the full partition function of the CFT is given by formula (4.7).

Let us now try to study the high temperature limit which corresponds to $\beta \rightarrow 0$ or $q \rightarrow 1$. We have

$$
\log Z(q)=\sum_{n=1}^{\infty} \frac{1}{n} \frac{q^{n \Delta}}{\left(1-q^{n}\right)^{d}}
$$

When $q \rightarrow 1$ the partition function goes like

$$
\log Z(q) \approx \frac{1}{(1-q)^{d}} \sum_{n=1}^{\infty} \frac{1}{n^{d+1}}
$$

or using $q=e^{-\frac{1}{T}}$

$$
\log Z(q) \approx \zeta(d+1) T^{d}
$$

where $\zeta$ is the Zeta function. Now we consider the free energy $F$ defined by $\log Z=$ $-F / T$. Reinstating the dependence on the radius $R$ of the $\mathbf{S}^{d-1}$ sphere we find that at high temperatures

$$
F \approx-\zeta(d+1) R^{d} T^{d+1}
$$

This result seems problematic in two (related) ways: first it is not extensive since it does not scale with the volume $R^{d-1}$ and second the $T$ dependence corresponds to that of a $d+1$ dimensional gas, while the CFT lives in $d$ dimensions! By contrast conformal invariance in $d$ spacetime dimensions implies that at very high temperatures $F$ must be proportional to $T^{d}$ on dimensional grounds. ${ }^{50}$ This suggests that the GFF Fock space is inconsistent with the basic assumptions of a local $d$-dimensional conformal field theory.

Notice that this problem does not arise for a standard free field of $\Delta=\frac{d-2}{2}$. Such a field obeys a $d$-dimensional equation of motion $\nabla^{2} \mathcal{O}=0$ on the boundary, so the derivatives are not all independent i.e. they obey the relation $\partial_{i} \partial^{i}=0$, so the equivalent of (4.8) is $Z_{1}(q)=\frac{q^{\frac{d-2}{2}}}{(1-q)^{d}}-\frac{q^{\frac{d+2}{2}}}{(1-q)^{d}}$, where we subtracted a term to compensate for operators which are zero due to the equations of motion. Effectively there is one less dimension for a field of $\Delta=\frac{d-2}{2}$ than one with $\Delta>\frac{d-2}{2}$ and the free energy of the former grows like $T^{d}$, which is consistent with the expectations for a $d$-dimensional CFT.

Going back to generalized free fields with $\Delta>\frac{d-2}{2}$, clearly one of our assumptions must be wrong. Namely, as we will argue shortly, the spectrum cannot have the structure

\footnotetext{
${ }^{50}$ Here we are assuming that in the high temperature limit, the leading term of the energy density on the sphere behaves like the thermal energy density on the plane.
} 
of a freely generated Fock space for arbitrarily large operators. In other words, a generalized free field cannot exist in an exact sense in a consistent CFT since it has the wrong thermodynamic properties.

We will see that there are two important ways that the spectrum of the theory has to be modified at large conformal dimension: first, we must truncate the growth of multiparticle states and, second, a new sector of states will have to be added to the theory, which are the analogue of black hole microstates.

Before we proceed let us notice that the same argument about the growth of the free energy with $T$ can be used to show that a non-gravitational theory in AdS (for example a free scalar field in AdS) cannot have a holographic dual CFT [84]. The free energy of a free hot gas in AdS is the same as (4.9). In order for this theory to have a holographic dual CFT the growth of the spectrum with $T$ has to be truncated by some mechanism in order to be consistent with that expected from a CFT i.e. $F \sim T^{d}$ and not (4.9). In gravitational theories this is achieved by gravitational collapse and the formation of black holes at high energies. See also [81] for an interesting related discussion in 2-dimensional CFTs.

\subsection{Decoupling of the stress tensor and the need for large $c$}

We now present another reason why a generalized free CFT cannot be a consistent theory by itself. A special operator present in any local conformal field theory is the stress energy tensor $T_{\mu \nu}$. It is an operator of dimension $\Delta=d$ transforming in the traceless symmetric 2-tensor representation of $\mathrm{SO}(d)$. Dropping Lorentz indices for simplicity, the 2-point function of the stress energy tensor has the following general form

$$
\langle T(x) T(y)\rangle=\frac{c}{|x-y|^{2 d}}
$$

where the constant $c$ plays the role of a "central charge" of the CFT. ${ }^{51}$

Recall that the OPE of $T_{\mu \nu}$ with a primary operator $\mathcal{O}$ is fixed by the Ward identities [65]. In other words the following 3-point function is exactly determined by the conformal dimension of $\mathcal{O}$ to be

$$
C_{T \mathcal{O O}} \sim\left\langle T_{\mu \nu} \mathcal{O O}\right\rangle \sim \Delta\langle\mathcal{O O}\rangle
$$

up to convention-dependent factors of order 1 . This means that when we consider the 4-point function of $\mathcal{O}$ and expand it in conformal blocks there should be a contribution from the block of the stress energy tensor. The coefficient with which this conformal block contributes is proportional to $\Delta^{2} / c$. More precisely the overall contribution from the stress tensor exchange to the 4-point function is

$$
\frac{\Delta^{2}}{c} \mathbf{G}_{T}\left(x_{1}, x_{2}, x_{3}, x_{4}\right)
$$

where the function $\mathbf{G}_{T}$ is completely fixed by conformal invariance (see [66] for the explicit expression of $\mathbf{G}_{T}$ in $d=4$ ). So as long as $\Delta>0$ this contribution cannot be zero or

\footnotetext{
${ }^{51}$ Here we are not careful about factors of order one in the normalization of $c$ since our arguments are qualitative. The precise relation between the constant in the 2-point function of the stress tensor and the conformal anomaly $c$ can be found in [65].
} 
cancelled by anything else. However a simple analysis of the factorized correlator (4.3) shows that no such conformal block appears! How is this possible? The only explanation is if we assume that the contribution (4.10) vanishes because $c=\infty$.

For a given and fixed CFT it does not make a lot of sense to assume that $c=\infty$. What is more reasonable is to understand this as a limiting sequence of theories for which $c \rightarrow \infty$. So a generalized free CFT can only be understood as a sector in a sequence of theories of ever increasing $c$.

In general $c$ is related to the number of degrees of freedom of the theory. ${ }^{52}$ Since $c \rightarrow \infty$ we expect a $c$-dependent growth in the number of degrees of freedom which is not apparent in the spectrum of a generalized free CFT, i.e. the free energy computed in section 4.3 was finite and $c$-independent. This implies that a generalized free CFT can only be a small part of a theory with a very large number of degrees of freedom, with the peculiar property that at low conformal dimension only a finite number of operators remain.

From now on and for the rest of the paper we will use the term generalized free CFT in the following sense: we assume that there is a sequence of CFTs of ever increasing central charge with the property that in the $c \rightarrow \infty$ limit a sector of a finite number of generalized free operators develops at low conformal dimensions. It may be more convenient to introduce a "coupling constant" $g$ such that when $c \rightarrow \infty$ we have $g \rightarrow 0 .{ }^{53}$ The coupling (4.10) to the stress tensor suggests that we define $g$ as

$$
g=\frac{1}{\sqrt{c}}
$$

Before we proceed let us see why the statement that factorization for GFFs implies $c \rightarrow \infty$ is consistent with the fact that correlators of ordinary free fields factorize even though the central charge is finite. For a genuinely free field of $\Delta=\frac{d-2}{2}$ there is a conformal block corresponding to the exchange of an operator with the quantum numbers of a "stress tensor" i.e. $\Delta=d$ and symmetric traceless, which is the two-particle operator $\left(: \partial_{\mu} \mathcal{O} \partial_{\nu} \mathcal{O}\right.$ : $+\cdots)$ where the dots denote the quadratic terms which appear in the stress tensor of a conformally coupled scalar. Of course this is not the stress tensor of the full CFT but only of the part of the CFT consisting of the free field $\mathcal{O}$, which is decoupled from the rest of the CFT since the field $\mathcal{O}$ is literally free. Notice that for a GFF with $\Delta>\frac{d-2}{2}$ the same two-particle operator has dimension greater than $d$ and thus it is not conserved.

\subsection{Gravitational collapse and "deconfinement"}

By now we have argued that while a generalized free CFT seems to satisfy the conformal bootstrap in flat space, it suffers from two pathologies: first if we assume that the Fockspace structure of the generalized free CFT persists all the way to arbitrarily high conformal

\footnotetext{
${ }^{52}$ In a weakly coupled CFT this is clear, since $T_{\mu \nu}$ couples to all fields and $c$ is proportional to the 2-point function of $T$ with itself. We give a few more arguments about the relation between $c$ and the degrees of freedom in section 7.1 .

${ }^{53}$ If the theory has more parameters than $c$, then we may have to tune them in an appropriate way in order to end up with a CFT satisfying factorization. For example in a gauge theory we may have to take the 't Hooft limit i.e. $N \rightarrow \infty, g_{Y M}^{2} N=$ const.
} 
dimension then the growth of states in the theory is not consistent with the one expected for a $d$-dimensional local CFT. Second, we found that for correlators to factorize we have to be able to decouple the stress energy tensor for which we have to assume that $c \rightarrow \infty$, in an appropriate limiting sense. The central charge $c$ is related to the degrees of freedom of the theory, so if $c \rightarrow \infty$ one would expect a $c$-dependent proliferation of states which seems not to take place for a generalized free CFT.

The most natural way to avoid these problems (suggested by what happens in large $N$ gauge theories) is to assume that a generalized free CFT is a "light" (in the sense of low conformal dimension) decoupled sector of a much bigger conformal field. If we probe the generalized free CFT at sufficiently high conformal dimension we will discover two things: that the multiparticle Fock space is truncated and that a new big sector of states (with $c$-dependent entropy) will kick in. The truncation of the multiparticle spectrum can be understood as the analogue of gravitational collapse while the new states are the "black hole" microstates.

That the free Fock space structure of a generalized free CFT has to be truncated at large conformal dimension can be understood as a dynamical effect in the following way: we saw that by taking $c \rightarrow \infty$ we can decouple the stress energy tensor. What happens though, if we look at correlators of operators with conformal dimension of the order $\Delta \sim c$ at the same time that we take the $c \rightarrow \infty$ limit? Then it is clear that the $\frac{1}{c}$ suppression in equation (4.10) is compensated by the $\Delta$ in the numerator and the conformal partial wave of the stress energy tensor cannot be ignored even if $c \rightarrow \infty$. This means that correlators of operators with conformal dimension of order $c$ cannot be factorizable.

More generally let us consider a many-particle state : $\mathcal{O O} \ldots \partial \mathcal{O} \ldots \partial \partial \mathcal{O}$ : of total (naive) conformal dimension $\Delta_{0}$, as computed by the Fock-space counting of conformal dimensions for multi-particle states in a generalized free CFT. If we consider the contribution from the exchange of the stress energy tensor between various constituents of the multiparticle operator, we find that it will inevitably introduce a correction $\delta \Delta$ to the naive conformal dimension $\Delta_{0}$. When the correction is of the same order as the naive dimension i.e. when $\delta \Delta / \Delta_{0} \sim 1$ then the freely generated Fock-space structure will definitely not be reliable. This happens when $\Delta_{0} \sim c$. Notice that this can happen even if the operator $\mathcal{O}$ has low conformal dimension, provided that we take sufficiently many insertions of $\mathcal{O}$ or $\partial$. Thus if we take sufficiently big multi-particle operators they will deviate from the free-Fock space no matter how large $c$ is.

In the bulk the equivalent statement is that if we take a gas of particles of total mass $M$ spread in a volume of the AdS radius, then the gravitational correction to their energy is of the order $G_{N} M^{2} / R_{\mathrm{AdS}}^{d-2}$, where $G_{N}$ is the bulk Newton's constant. In the limit $G_{N} R_{\text {AdS }}^{1-d} \rightarrow 0$ (which is equivalent to $c \rightarrow \infty$ ) the backreaction is negligible as long as $M$ is kept fixed. If however we scale $M \sim G_{N}^{-1} R_{\mathrm{AdS}}^{d-2}$ then we find that the backreaction is of the same order as the bare mass $M$ even in the $G_{N} \rightarrow 0$ limit. Such states cannot be made arbitrarily big because at some point they will hit their "Chandrasekhar" bound and will undergo gravitational collapse towards a black hole. See $[85,86]$ for related discussions.

To summarize we do not expect the spectrum of such large operators to arrange itself into a simple free Fock space as we naively assumed above. The effects of gravity (exchange 
of $T_{\mu \nu}$ in the boundary CFT ${ }^{54}$ ) cannot be ignored. Thus, we expect a modification of the simplistic multiparticle Fock space at conformal dimensions of order $c$ and a truncation due to "gravitational collapse".

What is more, in this regime (i.e. $\Delta>c$ ) we expect new sorts of states to emerge corresponding to black hole states. These states have a very different structure from the original multiparticle operators as their conformal dimensions and degeneracy is $c$ dependent (explaining why such states decouple in the limit $c \rightarrow \infty$ ). In particular, we expect the entropy of these new states to diverge as $c \rightarrow \infty$ (unlike the low-lying generalized free fields whose entropy is $c$ independent). This can be motivated as follows: from general arguments we expect that at very high temperature $T$ the expectation value of the stress tensor in a $d$-dimensional CFT goes like

$$
\left\langle T_{00}\right\rangle \sim \widetilde{c} T^{d}
$$

for some constant $\widetilde{c}$, from which we can derive that the entropy density is $S \sim \widetilde{c} T^{d-1}$. In section 7.1 we give some indications that $c \rightarrow \infty$ implies $\tilde{c} \rightarrow \infty$.

A natural question ${ }^{55}$ is whether these operators can be thought, in some sense, as multiparticle operators of GFF which, due to interactions, have received large corrections to their dimensions. For this, the corrections would have to be large enough so that even the qualitative scaling of their degeneracy with $c$ would be modified. In known examples, this would be analogous to assuming that there is some way to think of the quark-gluon plasma of a large $N$ gauge theory as a gas of glueballs with large corrections due to interactions, or a black hole as a strongly-interacting gas of gravitons. Both of these possibilities seem counter-intuitive. So perhaps it is more reasonable to assume that the spectrum at high conformal dimension corresponds to a genuinely new phase of the theory, in which new degrees of freedom emerge, and hence it is unreasonable to describe this high temperature phase in terms of excitations of the low temperature phase.

\subsection{Minimal generalized free CFT and gas of gravitons}

It is interesting to consider the smallest possible generalized free CFT, which would be the one whose only single particle operator is the stress energy tensor $T_{\mu \nu}$. As we argued, if we assume that in the $c \rightarrow \infty$ limit its correlators factorize then we necessarily have to add to the spectrum the multi-particle states made out of the stress energy tensor of the

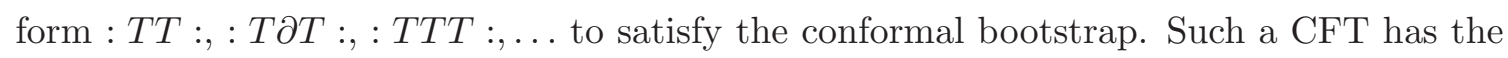
same spectrum as a linearized free graviton gas around AdS. As we explained before, such a CFT is inconsistent by itself and should be thought of as the low-conformal dimension sector of a much larger (sequence of) CFT with large $c$. In the bulk all these additional states would represent the black hole microstates. Such a theory was recently considered by E. Witten [76], for the case of three-dimensional AdS bulk. In two-dimensional CFTs the module of the stress energy tensor closes on itself and correlation functions of the stress tensor are completely fixed by holomorphy, with the only input being the value

\footnotetext{
${ }^{54}$ And all kinds of other operators of course, we only mention $T_{\mu \nu}$ because its coupling is universal.

${ }^{55} \mathrm{We}$ would like to thank J. de Boer for useful comments along these lines.
} 
of the central charge $c$ i.e. they do not contain any non-trivial dynamics. This is not the case for higher dimensional CFTs where the conformal group is not large enough to constrain the correlators of the stress energy tensor, which thus contain non-trivial dynamical information.

A natural question is whether such a minimal generalized free CFT is dual to (semi)classical gravity in the large $c$ limit. From the CFT point of view the question is whether the assumption that at $c=\infty$ the spectrum consists solely of the stress-tensor and multiparticle states is powerful enough to constrain the solutions of the bootstrap equations at order $1 / c$, to be those predicted by tree-level gravity. ${ }^{56}$ While this is a very interesting question we have not been able to say anything new about it, essentially because the CPW expansion of stress-tensor four-point functions is still not known.

Notice that according to our previous discussion one can also see that linearized gravity around AdS cannot be holographic unless the spectrum of (approximately) free gravitons is truncated at some point by gravitational interactions and the formation of black holes.

The stress tensor in dimension $d$ has $n=\frac{d(d+1)}{2}-1$ independent components and single particle states associated to it are

$$
T_{i j}, \partial_{i} T_{j k}, \partial_{i} \partial_{j} T_{k l}, \ldots
$$

The degeneracy at each level is $n$ times the degeneracy coming from the derivatives giving a (unconstrained) single-particle partition function

$$
\tilde{Z}_{1}(q)=\frac{n q^{d}}{(1-q)^{d}}
$$

Where we used that the conformal dimension of $T_{\mu \nu}$ is $d$. In any theory the stress-tensor satisfies the conservation equation $\partial^{i} T_{i j}=0$ which implies $d$ relations between derivatives. It is straightforward to find a generating function for these vanishing contributions (i.e. derivatives acting on $\partial^{i} T_{i j}$ )

$$
Z_{1}^{s}(q)=\frac{d q^{d+1}}{(1-q)^{d}}
$$

The corrected single particle partition function is the difference of these two

$$
Z_{1}(q)=\tilde{Z}_{1}(q)-Z_{1}^{s}(q)=\frac{q^{d}(n-d q)}{(1-q)^{d}}
$$

This is quite similar to the free scalar case above because $n>d$ for $d>2$ so in the limit $\beta \rightarrow 0$ we get

$$
Z_{1}(q)=\frac{n-d}{\beta^{d}}
$$

which will give a multi-particle partition function $\log Z(q) \approx T^{d}$ as in the generalized scalar case.

\footnotetext{
${ }^{56}$ Obviously one can write down bulk theories whose only light field is the graviton and which have higher derivative corrections. The question is to what extent such bulk theories can be related to a consistent boundary CFT.
} 


\subsection{Summary}

Let us review what we have discussed so far. We defined a "large $c$ generalized free CFT" by assuming it has a sector of "single-particle operators" $\left\{\mathcal{O}_{i}\right\}$ defined by the fact that their correlators factorize in an expansion in $1 / c$. Examining the bootstrap equations for such a theory we find that such fields cannot exist alone and in fact imply the existence of an entire tower of "multiparticle states" constructed from powers of $\mathcal{O}_{i}$ and derivatives.

At infinite $c$ the Hilbert space of such a "generalized free CFT" has the structure of a freely generated Fock space made out of the basic building blocks $\mathcal{O}_{i}$. Taking into account the finiteness of $c$ we concluded that such theories can only exist as small sectors of big conformal field theories.

The existence of the freely generated Fock space at infinite $c$ implies we are on the right track, as it is a necessary condition for the existence of a weakly coupled bulk dual. From the dual gravity point of view, the factorized nature of the large $c$ CFT is due to the fact that the correlators do satisfy a linear differential equation in $d+1$ dimensions and, as such, constitute a genuinely free theory implying a Fock space structure of the Hilbert space.

\section{Emergence of the holographic dimension}

We will now review arguments which indicate that when a large $c$ conformal field theory contains a sector of generalized free fields in its spectrum, then that sector and its interactions ${ }^{57}$ can most naturally be described by an effective higher dimensional theory which inevitably contains gravity. ${ }^{58}$ In this section we basically review statements made in $[5,6,8,87-90]$. We start with what happens in the free theory (i.e. at $c=\infty$ ).

A first hint towards the fact that a generalized free field should be represented in terms of an ordinary free field living in higher dimensions is the fact that the free energy of the former scales in the right way, according to the analysis of section 4.3. The free energy of a generalized free field is the same as that of a thermal gas of free particles living in $\mathrm{AdS}_{d+1}$.

A second important observation is that while the generalized free fields are in a sense "free", i.e. they generate a Fock space of excitations, they do not obey linear equations of motion on the boundary, which seems to be counter-intuitive. A freely-generated Fock space suggests that it is possible to superimpose excitations, which is a property of linear systems. The underlying linearity of a generalized free field can be made more manifest as follows: let us call $\mathbf{b}$ the $d$ Cartesian coordinates of the space on which the CFT lives and introduce a new set of $d+1$ auxiliary coordinates $(\mathbf{x}, z)$. We define the following family of operators

$$
\phi(\mathbf{x}, z)=\int d^{d} \mathbf{b} G(\mathbf{x}, z ; \mathbf{b}) \mathcal{O}(\mathbf{b})
$$

where $G(\mathbf{x}, z ; \mathbf{b})$ is a kernel, also called the "transfer function", which is defined by certain superposition of normalizable modes in AdS (see the references above for details). This

\footnotetext{
${ }^{57}$ When we move away from the strict $c \rightarrow \infty$ limit.

${ }^{58} \mathrm{By}$ this we do not necessarily mean "classical gravity" but also "stringy gravity" and other possible exotic versions.
} 
kernel satisfies the equation

$$
\left(\nabla_{d+1}^{2}-m^{2}\right) G(\mathbf{x}, z ; \mathbf{b})=0
$$

for a Laplacian defined on the manifold parametrized by the coordinates $(\mathbf{x}, z)$ and equipped with the $\mathrm{AdS}_{d+1}$ metric

$$
d s^{2}=\frac{d z^{2}+d \mathbf{x}^{2}}{z^{2}}
$$

Here $d \mathbf{x}^{2}=-d t^{2}+d x_{i}^{2}$ is in Lorentzian signature. The mass $m$ and conformal dimension $\Delta$ of the operator are related by the familiar formula

$$
\Delta=\frac{d}{2}+\sqrt{\frac{d^{2}}{4}+m^{2}}
$$

The operators $\phi(\mathbf{x}, z)$ are hermitian operators acting on the Hilbert space of the conformal field theory i.e. they are still operators "in the CFT", even though they are labeled by one additional parameter $z$. However they are not local operators. They are constructed by smearing local operators with the kernel $G(\mathbf{x}, z ; \mathbf{b})$.

The important property of the operators $\phi(\mathbf{x}, z)$ is that they satisfy a linear wave equation in the auxiliary $d+1$ dimensional manifold

$$
\left(\nabla_{d+1}^{2}-m^{2}\right) \phi=0
$$

Hence by lifting these operators in one additional dimension we can make the underlying linearity of the system manifest.

Correlation functions of the operators $\phi$ can be computed using the correlators of $\mathcal{O}$. To the extent that the factorization of correlators of $\mathcal{O}$ on the boundary is obeyed, we can see that correlators of the field $\phi$ in the "bulk" will be the same as those of a free massive field in AdS satisfying (5.5). For example the 2-point function of the field in the bulk can be computed by

$$
\left\langle\phi(\mathbf{x}, z) \phi\left(\mathbf{x}^{\prime}, z^{\prime}\right)\right\rangle=\iint d^{d} \mathbf{b} d^{d} \mathbf{b}^{\prime} G(\mathbf{x}, z ; \mathbf{b}) G\left(\mathbf{x}^{\prime}, z^{\prime} ; \mathbf{b}^{\prime}\right)\left\langle\mathcal{O}(\mathbf{b}) \mathcal{O}\left(\mathbf{b}^{\prime}\right)\right\rangle
$$

It is not hard to show using (4.2) that the 2-point function in the bulk computed this way coincides with the (bulk-to-bulk) Green's function of (5.5). In particular if this computation is performed in Lorentzian signature one finds that ${ }^{59}$

$$
\left[\phi(x, z), \phi\left(x^{\prime}, z^{\prime}\right)\right]=0
$$

if the two points are separated by a spacelike distance, as measured by (the Lorentzian version of) the metric (5.3). Hence the field (5.1) has the behavior of a local free field in AdS space.

What we have achieved so far is the following: we started with the $d$-dimensional CFT and we have introduced an emergent AdS space, which is used as a parameter space to

\footnotetext{
${ }^{59}$ For this equation to hold as an operator equation (i.e. to hold inside correlators with additional insertions) it is necessary to use the factorization of the correlators of $\mathcal{O}$.
} 
label operators of the form (5.1). What we have gained by this is that we have constructed a set of operators which obey linear equations of motion. In this sense the emergent bulk geometry makes the linearity of the theory more manifest.

The Fock space structure of the Hilbert space of the boundary conformal field theory can now be understood more directly as the standard Fock space of a free field in the bulk. The importance of this emergent AdS space will become more clear when we consider turning on small interactions.

Applying this procedure to the stress energy tensor, which is an operator present in any CFT, leads to a massless spin 2 field in the AdS space which implies that the effective holographic theory will be gravitational.

\subsection{Why this is not just group theory}

One might think that the association of an AdS space with a conformal field theory is somewhat trivial based on the identification of the isometry group of AdS with the conformal group in one less dimension. However this is not entirely correct. That the states in the Hilbert space of a CFT fall into representations of $\mathrm{SO}(2, d)$ is not sufficient to guarantee that they should have an interpretation as states living in a space with isometry group $\mathrm{SO}(2, d)$.

When we think of "emergent space" what we usually imagine is that we have an emergent manifold $M$ together with some quantum fields living on it (at least at long wavelengths). This means that the states should not only fall into representations of the isometry group of $M$, but also that the types and degeneracies of representations that appear in the Hilbert space must be consistent with those of (approximately) local quantum fields living on $M$.

According to our previous discussions the introduction of the dual spacetime is motivated by the fact that the low-lying Hilbert space of the CFT can be represented in terms of free (or weakly interacting) fields in the bulk, so it is important that the representations of $\mathrm{SO}(2, d)$ which appear in the spectrum of the CFT correspond to those of a free field in AdS. In particular they should have a Fock space structure and the correct extensive entropy. This requirement is satisfied for generalized free CFTs but would not be true for CFTs at finite central charge. For example it would not be true for the $\mathcal{N}=4$ SYM with gauge group, say, $\mathrm{SU}(2)$.

To summarize, the association of an AdS space ${ }^{60}$ to a CFT is not only based on group theory but also on the factorization of the low-lying operators, which is a dynamical assumption going beyond symmetry considerations.

\subsection{Operators with spin}

So far we have focused our discussion on scalar operators, but the same logic can be applied to operators of the CFT with spin. Generalized free operators of higher spin can

\footnotetext{
${ }^{60}$ As mentioned in section 2 , we restrict our attention to cases where there is at least some semi-classical notion of AdS space, which might be "stringy" but not "quantum", since we have no (independent) formulation of what quantum gravity means on an "AdS space" whose radius is comparable to the Planck scale.
} 
be associated to higher spin bulk fields. Everything we discussed in the previous subsection is readily generalizable.

Special care has to be taken if a generalized free operator of nonzero spin saturates the unitarity bound of the conformal field theory. In that case the boundary operator satisfies first order equations ${ }^{61}$ (for example for a conserved current this would be the conservation equation). Notice that operators with nonzero spin at the unitarity bound behave qualitatively differently from scalar operators at the unitarity bound, which satisfy second order equations (4.1).

The difference is that the first-order equations at the unitarity bound of operators with spin do not imply that the operator is "free" in any sense. For example, consider a conserved current $J^{\mu}$ in a 4 -dimensional CFT. It is an operator of $\Delta=3$ and $l=1$ which saturates the unitarity bound and obeys the first order equation $\partial_{\mu} J^{\mu}=0$. Unlike the second order equation $\nabla^{2} \mathcal{O}=0$ at the unitarity bound for a scalar field of $\Delta=1$, the first order conservation equation for a current does not allow us to compute correlation functions of $J_{\mu}$ with itself. ${ }^{62}$ In particular the correlators of $J_{\mu}$ with itself do not factorize to products of 2-point functions. In other words the current $J_{\mu}$ is not a generalized free field, even though it satisfies the first order equation $\partial_{\mu} J^{\mu}=0$. It is a genuinely interacting field.

If we now impose the additional assumption that a conserved current is "generalized free" (in the sense that its correlators factorize) then we have the following situation: as before, we try to construct "bulk" fields by applying a procedure similar to (5.1). The goal is to construct bulk fields which obey standard second order equations of motion. In the process of doing this the first order conservation equations on the boundary play important role. The resulting bulk fields have gauge invariance. In the case of a generalized free current on the boundary we get a free U(1) gauge field in the bulk, and for the case of the stress tensor we get a bulk spin two field with the gauge invariance of a linearized graviton. This is the well known relation between global symmetries in the CFT (which emerge when currents hit the unitarity bound) and gauge symmetries in the bulk.

\subsection{Comments on bulk observables and background independence}

Before we close this section we would like to make some comments about the construction of the bulk observables. While we can always define the operator (5.1) as a (non-local) operator in the CFT, the fact that it obeys (5.5) and (5.6) is not an exact statement but only true at infinite $c$ keeping certain other "parameters" of the problem fixed. To be more precise, if we want (5.5) and (5.6) to be satisfied as operator equations, then they must hold even when they are inserted in correlation functions evaluated on states of the CFT. If these states have a large enough mass to backreact on the geometry (i.e. if they have energy of order $c$ ) then we do not expect (5.5) and (5.6) to be true.

To see an example where this issue arises, let us consider a CFT with classical gravity dual, which is placed at finite temperature. Then we expect that correlators of gauge in-

\footnotetext{
${ }^{61}$ Because the norm of the first conformal descendant vanishes at the unitarity bound.

${ }^{62}$ Of course the conservation equation constrains the form of the correlation functions of $J_{\mu}$ but it is not powerful enough to fully determine them, unlike the equation $\nabla^{2} \mathcal{O}=0$ for a scalar at the unitarity bound $\Delta=1$.
} 
variant operators can be approximated by evaluating gravity correlators on the background of an AdS-Schwarzschild black hole. This means that if we want to "uplift" the generalized free fields $\mathcal{O}$ off the boundary and into regular free fields in the bulk, for the purpose of computing finite temperature correlators, we should be using a different kernel in (5.1), namely the kernel corresponding to propagation on a black hole background.

From the boundary point of view the deviation from (5.5) and (5.6) in these situations can be intuitively understood as follows: these relations were derived from the assumption of factorization of correlators of the boundary fields. As we explained, factorization is not an exact statement but only true in a $1 / c$ expansion. If we evaluate correlators on states with $c$-enhancing factors (for example if their conformal dimension is of order $c$ ) then the $1 / c$ suppression of interactions will no longer hold.

Moreover (5.6) may receive corrections if the points $x, x^{\prime}$ are brought very close to each other, that is, close enough so that the $1 / c$ suppression of interactions can be compensated. This means that the observables (5.1) are not infinitely localizable in the "bulk" unless we take $c \rightarrow \infty$ first.

The conclusion from this is the following: the effective bulk theory which we introduced in order to make manifest the linearity of the generalized free fields is not static and fixed but rather dynamical, in the sense that it depends on the saddle point/state of the CFT on which one is evaluating correlation functions. This is consistent with our expectation about a gravitational theory not having well defined (background independent) local observables.

It seems quite nontrivial that (at least in theories with classical gravitational duals) there are many classes of states/ensembles of the CFT on which correlators of operators can be easily computed by evaluating correlators of free fields propagating in some dual classical geometry which has to obey certain equations of motion ${ }^{63}$.

\section{$6 \quad$ Including interactions}

In section 5 we constructed operators in the generalized free CFT which behave like free fields in an emergent AdS space. The factorized correlators of this generalized free CFT have to be understood as the limiting form of correlators in a sequence of CFTs where $c \rightarrow \infty$. If we move a little bit away from the strict $c \rightarrow \infty$ limit we expect that the boundary CFT is no longer "generalized free", i.e. correlators of single particle operators no longer factorize. This indicates that there are certain effective ${ }^{64}$ interactions between them. We want to understand to what extent we can represent these interactions as local interactions in the emergent bulk space.

More specifically, our goal is to argue that the $1 / c$ expansion of a generalized free CFT can be naturally organized in terms of structures which coincide with "Witten diagrams" in anti de Sitter space.

\footnotetext{
${ }^{63}$ i.e. the Einstein equations and generalizations.

${ }^{64}$ Not to be confused with the interactions of the underlying fundamental fields of the theory which may be strong even in the strict $c \rightarrow \infty$ limit.
} 


\subsection{Scaling of correlators with $c$}

We now wish to consider the leading finite $c$ corrections. Thus we expand the CFT data $\left(\Delta_{i}, C_{i j}^{k}\right)$ in the small "coupling constant" $g \equiv \frac{1}{\sqrt{c}}$ around the point $g=0$. The choice of $\frac{1}{\sqrt{c}}$ as the expansion parameter is suggested by the coupling of the stress-tensor to other operators. If we rescale the stress tensor so that its 2-point function is order 1, i.e if we define $\widetilde{T}=\frac{1}{\sqrt{c}} T$, then the Ward identities fix the coupling to any other conformal primary to be of the form $\langle\widetilde{T} \mathcal{O O}\rangle \sim \frac{1}{\sqrt{c}} \Delta$, where $\Delta$ is the conformal dimension of $\mathcal{O}$. This suggests that the natural expansion parameter is $\frac{1}{\sqrt{c}}$, though one can imagine more complicated situations where various sectors of the CFT have different effective coupling. Since we want to focus on qualitative aspects we will ignore such complications and, in fact, much of what we say below is insensitive to the origin of the $g>0$ perturbation.

The corrections to the CFT data will thus take the form

$$
\begin{aligned}
\Delta_{i} & =\Delta_{i}^{(0)}+g \Delta_{i}^{(1)}+g^{2} \Delta_{i}^{(2)}+\ldots \\
C_{i j}^{k} & =C_{i j}^{k(0)}+g C_{i j}^{k(1)}+g^{2} C_{i j}^{k(2)}+\ldots
\end{aligned}
$$

These corrections to the conformal dimensions and to the OPE coefficients must be such that the bootstrap conditions are satisfied order by order in $g$.

To proceed let us first define the connected correlators $\left\langle\mathcal{O}_{i_{1}}\left(x_{1}\right) \ldots \mathcal{O}_{i_{n}}\left(x_{n}\right)\right\rangle^{c}$ of single trace operators by the following formal relation

$$
\begin{aligned}
\sum_{n=1}^{\infty} \int d x_{1} \ldots d x_{n} J_{i_{1}}\left(x_{1}\right) \ldots J_{i_{n}}\left(x_{n}\right)\left\langle\mathcal{O}_{i_{1}}\left(x_{1}\right) \ldots \mathcal{O}_{i_{n}}\left(x_{n}\right)\right\rangle \\
\quad=\exp \left(\sum_{n=1}^{\infty} \int d x_{1} \ldots d x_{n} J_{i_{1}}\left(x_{1}\right) \ldots J_{i_{n}}\left(x_{n}\right)\left\langle\mathcal{O}_{i_{1}}\left(x_{1}\right) \ldots \mathcal{O}_{i_{n}}\left(x_{n}\right)\right\rangle^{c}\right)
\end{aligned}
$$

Since 1-point functions vanish due to conformal invariance, we have for the 2 - and 3 -point functions

$$
\begin{aligned}
\left\langle\mathcal{O}_{1}\left(x_{1}\right) \mathcal{O}_{2}\left(x_{2}\right)\right\rangle^{c} & \equiv\left\langle\mathcal{O}_{1}\left(x_{1}\right) \mathcal{O}_{2}\left(x_{2}\right)\right\rangle \\
\left\langle\mathcal{O}_{1}\left(x_{1}\right) \mathcal{O}_{2}\left(x_{2}\right) \mathcal{O}_{3}\left(x_{3}\right)\right\rangle^{c} & \equiv\left\langle\mathcal{O}_{1}\left(x_{1}\right) \mathcal{O}_{2}\left(x_{2}\right) \mathcal{O}_{3}\left(x_{3}\right)\right\rangle
\end{aligned}
$$

The connected 4-point function is

$$
\begin{aligned}
\left\langle\mathcal{O}_{1}\left(x_{1}\right) \mathcal{O}_{2}\left(x_{2}\right) \mathcal{O}_{3}\left(x_{3}\right) \mathcal{O}_{4}\left(x_{4}\right)\right\rangle^{c} \equiv\left\langle\mathcal{O}_{1}\left(x_{1}\right) \mathcal{O}_{2}\left(x_{2}\right) \mathcal{O}_{3}\left(x_{3}\right) \mathcal{O}_{4}\left(x_{4}\right)\right\rangle \\
-\left\langle\mathcal{O}_{1}\left(x_{1}\right) \mathcal{O}_{2}\left(x_{2}\right)\right\rangle\left\langle\mathcal{O}_{3}\left(x_{3}\right) \mathcal{O}_{4}\left(x_{4}\right)\right\rangle \text { - permutations }
\end{aligned}
$$

and so on.

In order to proceed we will make the simplifying assumption that the connected correlators of single-particle operators scale as

$$
\left\langle\mathcal{O}_{i_{1}}\left(x_{1}\right) \ldots \mathcal{O}_{i_{n}}\left(x_{n}\right)\right\rangle^{c} \sim g^{n-2}
$$

plus subleading corrections, which scale with higher powers of $g \sim \frac{1}{\sqrt{c}}$. From the CFT point of view there is no a priori reason to assume this specific scaling, but we choose it as it is 
the one corresponding to theories that we are familiar with and leads to simple results. ${ }^{65}$ It would be interesting to study which of our following statements will be modified if a different scaling is assumed. The scaling (6.7) holds in the usual large $N$ expansion of gauge theories [28] (where $c \sim N^{2}$ and thus $g \sim \frac{1}{N}$ ) and in large $N$ symmetric orbifold CFTs in two dimensions [36-38]. From a string theory point of view this scaling means that each time we add a vertex operator to a genus zero diagram we get a power of the string coupling constant which in this case would be identified with $g$.

In the rest of this paper we will only discuss the leading terms of the connected correlators (6.7) which should be thought of as tree level correlators of the dual AdS theory. We will make the crucial assumption that at this order the conformal bootstrap can be solved purely within the low-lying generalized free field sector. In other words we will assume that to leading order in the $g$ expansion, there are no operators dual to black hole microstates running in the decomposition of the correlators of light fields. It would be interesting to analyze in more detail what constraints this assumption implies about the degeneracies and couplings of heavy operators and also how this condition is modified at higher orders in the $g$-expansion.

The scaling assumption (6.7) basically fixes the $g$ scaling of various OPE coefficients. For example from (6.7) we find that the OPE coefficient between three single trace operators starts at order $g=\frac{1}{\sqrt{c}}$. Similarly the OPE coefficient between two single particle states $\mathcal{O}_{1}, \mathcal{O}_{2}$ and the composite two-particle operator : $\mathcal{O}_{1} \mathcal{O}_{2}$ : starts at order 1 (from the disconnected correlator) and the next correction can start at order $g^{2}=\frac{1}{c}$, as indicated by the scaling of the connected correlator $\left\langle\mathcal{O}_{1} \mathcal{O}_{1} \mathcal{O}_{2} \mathcal{O}_{2}\right\rangle$. The leading terms of the OPE of two single particle operators $\mathcal{O}_{1}(x)$ and $\mathcal{O}_{2}(0)$ will have the following schematic form

$$
\mathcal{O}_{1} \mathcal{O}_{2} \sim \frac{1}{x^{\Delta_{1}+\Delta_{2}}}\left[g \mathcal{O}_{k} x^{\Delta_{k}}+\left(\delta_{i\{1} \delta_{2\} j}\left(1+g^{2} \log x\right)+g^{2}\right) \mathcal{O}_{n, l}^{(i j)} x^{\Delta_{i}+\Delta_{j}+2 n+l}\right]
$$

So a consequence of (6.7) is the vanishing of the order $g$ terms in the multiparticle contribution to the single-particle OPE. Note we only indicate the $g$-dependence above; in principle each term has a free coefficient. The logarithmic terms arise from corrections to the conformal dimensions of the two-particle operators.

\subsection{Conformal bootstrap in perturbation theory and holographic interactions}

We now wish to study solutions of the bootstrap equations perturbatively in $g$ around the generalized free field solution. Our goal is to argue that such a perturbation is most naturally described in terms of Witten diagrams in the AdS space introduced in the previous section.

First of all we consider the converse problem: i.e. we start with the free bulk theory constructed in section 5 and consider a perturbation by adding local interactions in the bulk. If we assume that correlators on the boundary are still related to correlators in

\footnotetext{
${ }^{65}$ One can construct examples with more complicated scaling, for example by taking products of CFTs with different ranks of the gauge groups and turning on interactions between them. Our goal is not to consider the most general case, but rather to find examples which capture the essential physics without adding unnecessary complications.
} 

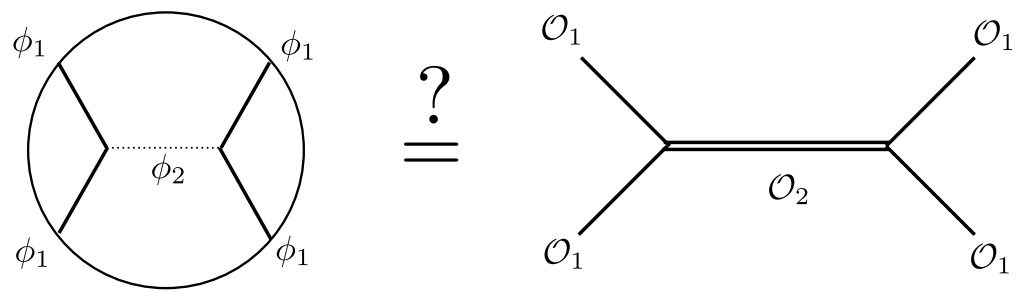

Figure 4. Witten diagram and conformal partial wave.

the bulk by the usual AdS/CFT prescription then the perturbed CFT correlators will be related to "Witten diagrams" in the bulk [5]. For example turning on an interaction of the form $\int d^{d+1} x \sqrt{g} \phi_{1} \phi_{2} \phi_{3}$ will correspond to a nonzero boundary 3-point function $\left\langle\mathcal{O}_{1} \mathcal{O}_{2} \mathcal{O}_{3}\right\rangle$, where the three operators $\mathcal{O}_{1}, \mathcal{O}_{2}, \mathcal{O}_{3}$ on the boundary dual to fields $\phi_{1}, \phi_{2}, \phi_{3}$ in the bulk.

From the symmetries of the problem it is clear that if the interacting correlators are computed from Witten diagrams they will automatically satisfy some of the consistency requirements of the CFT, such as having an expansion consistent with an OPE and crossing symmetry. This does not imply full consistency from the CFT point of view, as one still has to check the unitarity of the theory. In other words a Witten diagram expansion does not guarantee that the OPE coefficients that will be computed from Witten diagrams will necessarily be real (or their square positive). ${ }^{66}$ Of course it would be extremely interesting to use this approach to find constraints on possible effective actions in AdS spaces, but we will not do it in this paper (but see e.g. [12]).

The logic of our approach is the opposite and we want to understand the emergence of the interactions in the bulk starting from the CFT. In other words we want to explain why a physicist studying the perturbations of a "generalized free CFT" would naturally introduce the concept of a "Witten diagram". From the CFT point of view interactions between operators are naturally described in a conformal partial wave expansion. To understand why in generalized free CFTs this expansion can be reorganized in terms of Witten diagrams we have to clarify the precise relation between conformal partial waves and Witten diagrams.

Let us consider a basic exchange Witten diagram with 4 external $\phi_{1}$ lines and the exchange of a particle of type $\phi_{2}$. Intuitively one would expect that it is dual to the conformal block corresponding to the exchange of the operator $\mathcal{O}_{2}$ (and all of its descendants) between four external $\mathcal{O}_{1}$ operators, as depicted in figure 3 . However a careful comparison of the two shows that they are not exactly the same [91]. The exchange Witten diagram can be explicitly evaluated and expanded in a basis of conformal blocks. One then finds

\footnotetext{
${ }^{66}$ For example consider a 4-point function of single trace operators $\left\langle\mathcal{O}_{1}\left(x_{1}\right) \mathcal{O}_{2}\left(x_{2}\right) \mathcal{O}_{1}\left(x_{3}\right) \mathcal{O}_{2}\left(x_{4}\right)\right\rangle$ which we compute by using effective Witten diagrams in the bulk. Let us say that in the channel (12) $\rightarrow(34)$ a single trace operator $\mathcal{O}_{3}$ appears. The corresponding CPW comes multiplied by the coefficient $p=\left(C_{12}^{3}\right)^{2}$, which is of order $1 / c$. Unitarity implies that $p$ must be positive, which (presumably) imposes certain constraints on the effective Witten diagrams used to compute the 4-point function. In [10] constraints from unitarity were not relevant because they only considered the exchange of two-particle operators in the intermediate channel, which means that the relevant constraints would only appear at higher order in $1 / \sqrt{c}$.
} 
that it is equal to the conformal block of the exchange of $\mathcal{O}_{2}$ plus additional conformal blocks corresponding to the exchange of 2-particle states of the form : $\mathcal{O}_{1} \partial \ldots \partial \mathcal{O}_{1}:$. The exchange of these operators is not represented by additional diagrams in the bulk, instead they are already encoded in the basic exchange Witten diagram.

This raises the questions: what is the precise relation between Witten diagrams and conformal blocks? What is the role of these additional 2-particle states? Why are they automatically included in the Witten exchange diagram? We will address these questions in the next subsection. Notice that the relation between Witten diagrams and conformal blocks has been discussed in several works in the past [66, 91-99].

\subsection{CFT interpretation of Witten diagrams: "dressed" conformal blocks}

These questions were partly addressed by the authors of $[10,11]$. They considered the 4-point function of an operator $\mathcal{O}$ in an $\frac{1}{N}$ expansion and showed that the solutions of crossing symmetry, under certain technical assumptions about the spins of the intermediate fields, are in one-to-one correspondence with the possible contact Witten diagrams that can be written in the bulk for the dual field $\phi$. This provides evidence that the tree level interactions of the generalized free field $\mathcal{O}$ can be described in terms of an effective action in AdS for the dual field $\phi$.

Here we develop a related perspective by focusing on the CFT interpretation of the scalar exchange Witten diagrams. Our goal is to explain why, in perturbed generalized free CFTs, it is more natural to reorganize the $\frac{1}{c}$ expansion into an expansion in certain linear combinations of conformal partial waves. These linear combinations of conformal partial waves coincide with the Witten diagrams. Hence we will try to argue that even if we did not know about AdS/CFT, we would still have a motivation to introduce the Witten diagrams in order to describe the perturbative expansion of generalized free CFTs.

The main point is that a single conformal partial wave, once expanded in the crossed channel, has undesirable behavior: it cannot be written as a superposition of conformal blocks in the crossed channel, consistently with the scaling assumption (6.7) for the perturbed correlators. We will explain this point below.

Of course this is not a problem in principle, since we are supposed to solve the bootstrap conditions by summing over all conformal partial waves in the direct channel and writing this sum in terms of another sum of conformal partial waves in the crossed channel. That is, the bootstrap conditions apply to the sums and not to individual terms. However, relating two infinite sums over conformal partial waves makes solving the bootstrap equations difficult and it would be useful if we could break down the problem into smaller pieces. In particular it would be helpful if we could redefine the conformal blocks in such a way that each individual "redefined block" had nice factorization properties in all channels, consistent with the large $c$ scaling. If that was possible we could then combine these redefined blocks to get the most general solution of the bootstrap equations. As we will argue, this is possible for generalized free CFTs, and these "redefined blocks" are precisely the Witten diagrams.

We will now explain how this works in the case of a 4-point function and we will discuss higher order correlators in a later subsection. We consider the correlation function of four 
single-particle scalar operators

$$
\left\langle\mathcal{O}_{1}\left(x_{1}\right) \mathcal{O}_{2}\left(x_{2}\right) \mathcal{O}_{3}\left(x_{3}\right) \mathcal{O}_{4}\left(x_{4}\right)\right\rangle
$$

To avoid certain technical complications we assume that the conformal dimensions are different generic real numbers. ${ }^{67}$ This implies that at leading order (order $g^{0}$ ) the correlator vanishes, so we do not have to deal with disconnected contributions to the perturbed correlator. According to our scaling assumption the 4-point function will become nonzero at order $g^{2}$. Moreover we will assume that the conformal dimensions of the single-trace operators do not receive any corrections as we turn on $g$. This is a harmless simplifying assumption in order to avoid technical complications. It is true, for example, for chiral primaries in supersymmetric CFTs. ${ }^{68}$

Let us consider the conformal partial wave $\mathbf{G}_{i}^{12,34}$, which was defined in (3.5), corresponding to the exchange of the operator $\mathcal{A}_{i}$ and its descendants in the channel (12) $\rightarrow(34)$ and the conformal partial wave $\mathbf{G}_{j}^{14,23}$ for the exchange of an operator $\mathcal{A}_{j}$ in the channel $(14) \rightarrow(23)$. The bootstrap condition is

$$
\sum_{i} C_{12}^{i} C_{34}^{i} \mathbf{G}_{i}^{12,34}=\sum_{j} C_{14}^{j} C_{23}^{j} \mathbf{G}_{j}^{14,23}
$$

where $C_{i j}^{k}$ are the OPE coefficients.

This is a complicated equation for the OPE coefficients $C_{i j}^{k}$. How can we solve it? In a general CFT the problem is too difficult and, apart from free (and generalized free) CFTs, has only been solved for certain simple two-dimensional CFTs. However, in a perturbed generalized free CFT we can use the assumption (6.7) to derive some additional information about the kind of operators which appear in the summation over $i$ and $j$. The OPE between $O_{1}$ and $O_{2}$ is constrained by the scaling (6.7) and has a low order expansion (in $g$ ) given in (6.8). At order $g^{0}$ we can only have 2-particle operators of the form : $O_{1} \partial \ldots \partial O_{2}$ : and : $O_{2} \partial \ldots \partial O_{1}$ : while at order $g$ we can only have new single particle operators $O_{i}$. At order $g^{2}$ we can have corrections to the coefficients of operators which appeared at lower order (including the order 1 coefficient) and new 2-particle operators of the form $: O_{i} \partial \ldots \partial O_{j}$ : with $(i, j) \neq(1,2)$. In particular we can have 2-particle operators of the form : $O_{3} \partial \ldots \partial O_{4}:$. Similar results are true for the OPEs in the crossed channel. ${ }^{69}$

Because of this scaling, when we consider the double OPE in the (12) $\rightarrow$ (34) channel we see that there is no possible contraction at order $g^{0}$ or $g^{1}$. At order $g^{2}$ we have two kinds of contractions: the order $g^{2}$ 2-particle operator on one side can contract with a leading order operator on the other side, or the order $g$ single-particle operators on both sides can

\footnotetext{
${ }^{67}$ i.e. no linear combination of the dimensions with integer coefficients is equal to an integer number. Otherwise logarithmic terms may appear due to corrections to the dimensions of double-trace operators.

${ }^{68}$ Though in this case it is more likely that the conformal dimensions will be integer numbers and thus logarithmic corrections will have to be considered.

${ }^{69}$ Notice that because we chose to use four different operators we do not have any contribution from the correction to conformal dimensions of double trace operators. While these corrections are in general nonzero, they enter at higher order in perturbation theory. This would not be the case if we had considered four-point functions of the same operators on the external legs.
} 
contract together. So the solution of the bootstrap equations has to be constructed by using only the operators mentioned above.

Using these operators we would like to find basic building blocks, i.e. basic solutions of the bootstrap equations, which we will then be able to combine to get more complicated solutions. Let us start with the exchange of just one single-particle operator $\mathcal{O}_{m}$ in the $(12) \rightarrow(34)$ channel, i.e. we take $C_{12}^{i}, C_{34}^{i} \sim \delta^{i m}$. Then the l.h.s. of (6.9) is equal to a single conformal block $\mathbf{G}_{m}^{12,34}$ in this channel. Now we ask whether we can choose the coefficients $C_{14}^{j}, C_{23}^{j}$ in such a way that the equation is solved.

For this let us try to see whether a single conformal block in the direct channel can be expanded in the basis of conformal blocks in the crossed channel as

$$
\mathbf{G}_{m}^{12,34}=? \sum_{n, \text { allowed }} K_{m n} \mathbf{G}_{n}^{14,23}
$$

where $K_{m n}$ is a matrix to be determined. While trying to solve this equation we additionally have to make sure that the operators labeled by $n$, running in the crossed channel, are consistent with the assumptions about the OPE between (13) and (24) i.e. that they are only single-particle operators at order $g$ and double-particle operators at orders $g^{0}$ and $g^{2}$. With these additional restrictions it is easy to check that it is not possible to write a single conformal block in the direct channel in terms of the allowed blocks in the crossed channel i.e. it is not possible to find $K_{m n}$ which satisfies (6.10). ${ }^{70}$

This means that if we start with a single conformal block in the direct channel, we have to "dress it up" with other allowed 2-particle operators to make it have a nice (i.e. consistent with the OPE and the scaling (6.7)) expansion in the crossed channel.

This can be done in more than one ways, but we would like to find the minimal modification of a basic conformal block so that it can solve the bootstrap conditions by itself. So we start with the conformal block $\mathbf{G}_{m}^{12,34}$ corresponding to the exchange of $m$ in the $(12) \rightarrow(34)$ channel and we add to it a superposition of other conformal blocks $\mathbf{G}_{n}^{12,34}$ corresponding to the exchange of other operators $n$ in the same channel, and we try to adjust this superposition in such a way that when expanded in the (14) $\rightarrow$ (23) channel it can be expressed as a superposition of the allowed (that is, allowed by the scaling (6.7)) operators. So we define the object

$$
\mathbf{W}_{m}^{12,34}\left(x_{1}, x_{2}, x_{3}, x_{4}\right)=\mathbf{G}_{m}^{12,34}\left(x_{1}, x_{2}, x_{3}, x_{3}\right)+\sum_{n \in \text { direct channel }} c_{m}^{n} \mathbf{G}_{n}^{12,34}\left(x_{1}, x_{2}, x_{3}, x_{4}\right)
$$

where the sum over $n$ runs only over the allowed operators ${ }^{71}$ in the direct channel. We want to choose the coefficients $c_{m}^{n}$ so that we can write

$$
\mathbf{W}_{m}^{12,34}\left(x_{1}, x_{2}, x_{3}, x_{4}\right)=\sum_{n \in \text { crossed channel }} d_{m}^{n} \mathbf{G}_{n}^{14,23}\left(x_{1}, x_{4}, x_{2}, x_{3}\right)
$$

\footnotetext{
${ }^{70}$ For example when the conformal block $\mathbf{G}_{m}^{12,34}$ is expanded in the crossed channel, it has logarithmic terms in the conformal cross-ratios. This logarithmic behavior is inconsistent with an OPE expansion in the crossed channel, since we argued that due to the large $c$ scaling assumption (6.7), and for 4-different operators on the external legs, there are no contributions of anomalous dimensions in the correlator at this order, which might introduce logarithmic dependence.

${ }^{71}$ i.e. allowed by the assumption (6.7) for the scaling with $g$ of the connected correlators.
} 
for appropriate coefficients $d_{m}^{n}$. Here the index $n$ runs over the allowed operators in the crossed channel. This is a problem for the unknown numbers $c_{m}^{n}, d_{m}^{n}$.

To find the simplest possible solution we first make the assumption that there is no other single particle operator involved in the sums, apart from $\mathcal{O}_{m}$ in the direct channel, but we can have 2-particle operators running in both channels, allowed by (6.7). Even after imposing these restrictions, there is still more than one solution to our problem. Motivated by [10], we try to look for the simplest possible solution by restricting the maximum spin of the 2-particle operators which are exchanged. If we demand that all 2-particle (primary) operators involved in the summations above are scalars then we drastically reduce the number of solutions of the bootstrap equations.

However even with these constraints the solution is still not unique. It turns out that with the constraints that we imposed in the last paragraph, there is still a one-parameter family of solutions. To understand more intuitively why there is an ambiguity let us consider the problem we are trying to solve: we assume the exchange of the operator $\mathcal{O}_{m}$ (and of its descendants) and ask how we can dress it up by adding contributions of 2particle scalar operators in such a way that the combination has nice factorization in the crossed channel. The ambiguity comes from the fact that there is actually a combination of the 2-particle states which by itself (without adding the contribution of $\mathcal{O}_{m}$ ) solves the bootstrap. This solution is the one corresponding to the basic contact Witten diagram in the bulk. The explicit expansion of the contact Witten diagram in terms of CPWs can be found in appendix B. This solution can be added with arbitrary overall coefficient to any other solution of the original problem, containing the exchange of $\mathcal{O}_{m}$. In a sense this is like trying to solve an inhomogeneous linear problem, where the solution is defined up to an ambiguity of adding any solution of the corresponding homogeneous problem.

As usual this ambiguity is fixed by imposing appropriate boundary conditions. The "homogeneous solution" (the solution involving only 2-particle scalar operators) is unique [10] and is exactly equal to the contact Witten diagram in the bulk. With appropriate boundary conditions, the "dressed conformal block" should become unambiguously defined and as we will see it will precisely coincide with the exchange Witten diagram.

What is the relevant "boundary condition" is not obvious a priori. We can guess what it should be by using the intuition from the Witten diagram picture: we have to identify a qualitative difference between an exchange and a contact diagram. One difference is that the contact diagram is "harder" at very high energies. In the case of the exchange diagram the propagator of the exchanged particle suppresses the diagram if the momentum of the intermediate particle is very large. Such a suppression does not take place in the contact diagram. In CFT language this difference can be translated into a statement about the singularity structure of the 4-point functions. This singularity becomes obvious in the Lorentzian continuation of the 4-point function. Since these points are somewhat technical they will be not be explained here but we refer the reader to the relevant papers $[10,100-$ 103]. The important point is that the 4-point function, as computed by tree level Witten diagrams, has a singularity as a function of the conformal cross ratios when there is a common point in the light-cones of the 4 operators. The strength of this singularity is qualitatively different between a contact and an exchange Witten diagram. 


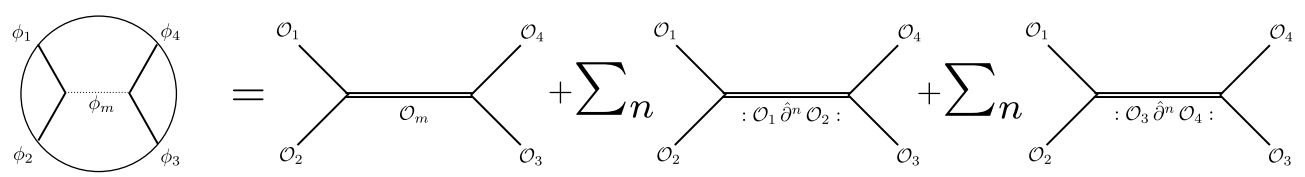

Figure 5. Conformal partial wave expansion of a scalar exchange Witten diagram in the direct channel. The coefficients of the various CPWs are computed in appendix B.

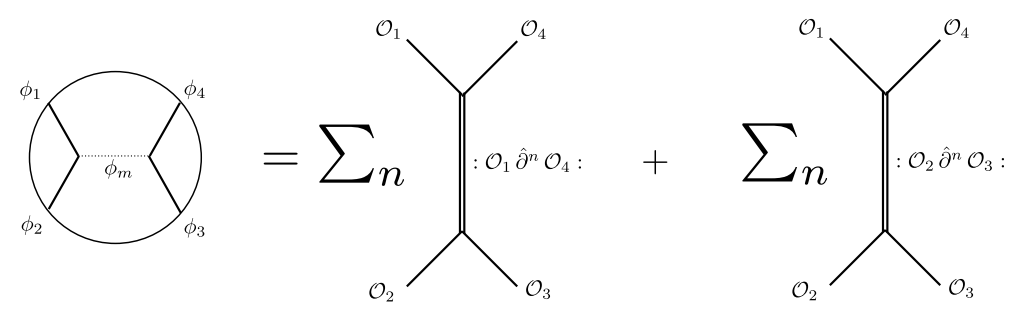

Figure 6. Conformal partial wave expansion of a scalar exchange Witten diagram in the crossed channel.

The conclusion is that if we demand that the solution has as soft a singularity as possible then it is unique: there is a unique way to dress up a conformal partial wave by 2-particle operators of spin zero so that it solves the bootstrap equation keeping the short distance singularity as soft as possible. This unique solution is the exchange Witten diagram.

\subsection{Summary: CFT interpretation of the basic exchange Witten diagram}

Let us review the previous section: the basic scalar exchange Witten diagram is equal to a single conformal block dressed up with 2-particle scalar operators in such a way that its expansion in the crossed channel is consistent with an OPE, large $c$ scaling, is as simple as possible (i.e. it only involves scalar 2-particle operators), and the singularity in the Lorentzian regime is as weak as possible. The explicit form of the 2-particle coefficients of this solution are expressed in appendix B.

Solutions of the same problem where we include higher spin intermediate 2-particle operators correspond to Witten exchange diagrams with derivative interactions (or can be reinterpreted as having mixing with contact Witten diagrams with derivative interactions [10], since there is always an ambiguity related to field redefinitions [91]). It would be interesting to demonstrate this more explicitly. Finally solutions of the problem where no single particle operator is involved at all (i.e. the "homogeneous" solutions mentioned above) correspond to contact Witten diagrams as demonstrated in [10].

Similar statements should hold for exchange diagrams of intermediate fields with spin, such as the graviton which is dual to the stress energy tensor. Though we have not checked this explicitly, we expect that the graviton exchange diagram ${ }^{72}$ is equal to the conformal partial wave of the stress-tensor exchange, dressed up with 2-particle states in such a way that the total contribution factorizes into allowed 2-particle states in the crossed channel.

\footnotetext{
${ }^{72}$ In Einstein-Hilbert gravity.
} 


\subsection{Dressing the OPE}

In the previous section we explored the utility of the bulk in providing a perturbative solution of crossing symmetry. The CFT data, however, is specified not by the conformal partial waves but rather by the OPE coefficients and conformal dimensions. Ideally then we would like to argue that bulk interactions provide a natural way to "dress" the OPE of a large $c$ CFT so that it manifestly satisfies crossing symmetry. This extends, to the interacting theory, the intuition that the bulk provides a manifest realization of the "weakcoupling" of the GFF.

Imagine we try to find a solution to crossing symmetry by perturbing some OPE coefficients of the factorized theory. For instance we can try turning on a non-trivial single-particle OPE $C_{12}^{k} \sim \mathcal{O}(g)$ for some $k$ and ask if this is a consistent deformation of the CFT. This deformation is, of course, constrained by crossing symmetry of $\left\langle\mathcal{O}_{1} \mathcal{O}_{2} \mathcal{O}_{1} \mathcal{O}_{2}\right\rangle$ but this constraint is difficult to analyze because corrections to the conformal dimension of $\mathcal{O}_{1} \mathcal{O}_{2}$ two particle states enter at leading order (ie. $g^{2}$ ) and generate logarithmic terms in the expansion.

In order to avoid this subtlety we consider simultaneously deforming both $C_{12}^{k}$ and $C_{34}^{k}$ (subject to the constraints on the conformal dimension mentioned in the previous section). From the previous section and appendix B we see that this, by itself, is not a consistent deformation of the CFT. Rather any such deformation must be accompanied by a deformation of $C_{12}^{: 34 n}$ : and $C_{34}^{: 12 n}$ : where : $12 n$ : and : $34 n$ : indicate multiparticle states of the form $\mathcal{O}_{1} \nabla^{n} \mathcal{O}_{2}$ and $\mathcal{O}_{3} \nabla^{n} \mathcal{O}_{4}$, respectively. These deformations must occur in a precise ratio given by comparing (B.7) and (B.8). Moreover, we must also deform $C_{14}^{: 23 n}$ :

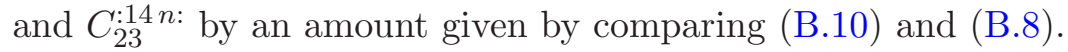

From (B.7)-(B.10) it is thus possible to extract a prescription for a one parameter family of consistent deformations of the GFF OPE coefficients. Of course from the bulk this is nothing more than the two parameter deformation given by $g_{12 k} \phi_{1} \phi_{2} \phi_{k}$ and $g_{34 k} \phi_{3} \phi_{4} \phi_{k}$ but the CFT is only constrained by the product $g_{12 k} g_{34 k}$ (we could extract constraints associated only to $g_{12 k}$ but we would have to consider $\left\langle\mathcal{O}_{1} \mathcal{O}_{2} \mathcal{O}_{1} \mathcal{O}_{2}\right\rangle$ or some more complicated combination of correlators).

This suggests that the OPE coefficients are somehow not the most natural basis to perturb a large $c$ CFT with but rather we would like to work with "dressed" coefficients $\tilde{C}_{12}^{k}$ which are particular linear combinations of the original OPE which correspond, in some sense, to a bulk three-point vertex. It may be that correlators in a large $c$ CFTs enjoy an alternate OPE expansion that manifestly solves crossing symmetry (perhaps not unrelated to the proposal of $[104]^{73}$ or that of [13]). In such an expansion the holographic nature of perturbed GFF correlators may well be more manifest.

As mentioned in the previous section, however, the above prescription or deformation, is not unique. We also have the freedom to switch on single-multiparticle interactions without any single-single particle interactions. We could e.g. switch on $C_{12}^{: 34}$ so long as we also switch on $C_{34}^{: 12:}, C_{14}^{: 23:}$ and $C_{23}^{: 14:}$ in a way constrained by the function $a_{n}$ of (B.3) (i.e. the ratio of these coefficients is fixed by the ratio of the $a_{n}\left(\Delta_{i}, \Delta_{j}, \Delta_{k}, \Delta_{l}\right)$ with its arguments

\footnotetext{
${ }^{73}$ We would like to thank S. Rychkov for bringing this paper to our attention.
} 
appropriately permuted). As a consequence of this freedom it is far from obvious that there is a natural way to modify the OPE expansion so that it corresponds to a bulk vertex expansion (as the former is always cubic while the latter can involve higher point vertices).

It would be interesting to explore this construction further but for now we wish merely to point out that the constraints coming from the CPW expansion enjoy a natural reformulation in terms of deformations of OPE coefficients. Note further that if one performs the minimal deformation (consistent with having only a three-point vertex in the bulk) this defines a consistent CFT including multi-particle correlators as well. More general solutions, however, are possible corresponding to introducing four and higher point vertices in the bulk.

\subsection{Conformal bootstrap for higher $n$-point functions}

We now sketch what happens when we consider higher $n$-point functions of single-particle operators, but still at tree level i.e. focusing on the leading terms in the scaling (6.7). Before doing so let us mention some basic facts. In a general conformal field theory the bootstrap conditions correspond to checking equation (3.6) for all primary operators on the external legs. For a CFT which is a perturbation of a "generalized free CFT" there is an alternative formulation of the bootstrap conditions: instead of checking that the 4point function of all conformal primaries factorizes correctly in all channels, we can check that the $n$-point function of single particle operators factorizes correctly in all possible OPE decompositions. ${ }^{74}$ In this way we have to check the factorization of correlators of fewer operators (only the single-particle ones) but the trade-off is that we have to consider all $n$-point functions instead of just the 4-point functions. Let us refer to this equivalent formulation as the "single-particle bootstrap".

If we check that the "single-particle bootstrap" is true for all single particle operators on the external legs, then we can show that the standard bootstrap is also satisfied for all possible multi-particle operators on the external legs of 4-point functions: this can be done by constructing the latter as a limit of the former. For example the factorization properties of a correlator of 4 two-particle operators can be derived by the factorization properties of a correlator of 8 single-particle operators by taking the limit of pairs of insertions coming together and subtracting singular terms. As we will see later the "single-particle bootstrap" condition is more natural to impose for a generalized free CFT. This is also suggested by the fact that Witten diagrams in supergravity are dual to correlators of single particle operators. ${ }^{75}$ This is again a special property we expect for CFTs with a gravity dual at large $c$; a generic CFT does not have a sub-basis of "single-particle" operators from which the full set of operators can be generated.

Let us explain in more detail what the "single-particle bootstrap" condition means. An $n$-point function of single-particle operators can be evaluated by performing successive OPEs between the operators. For this we need to choose an order in which to perform

\footnotetext{
${ }^{74}$ Of course this condition only guarantees the consistency of the low-lying sector of generalized free fields and not of the entire CFT

${ }^{75}$ The correlators of multi-particle operators can be computed in AdS/CFT by taking limits of higherpoint Witten diagrams of single-particle operators.
} 


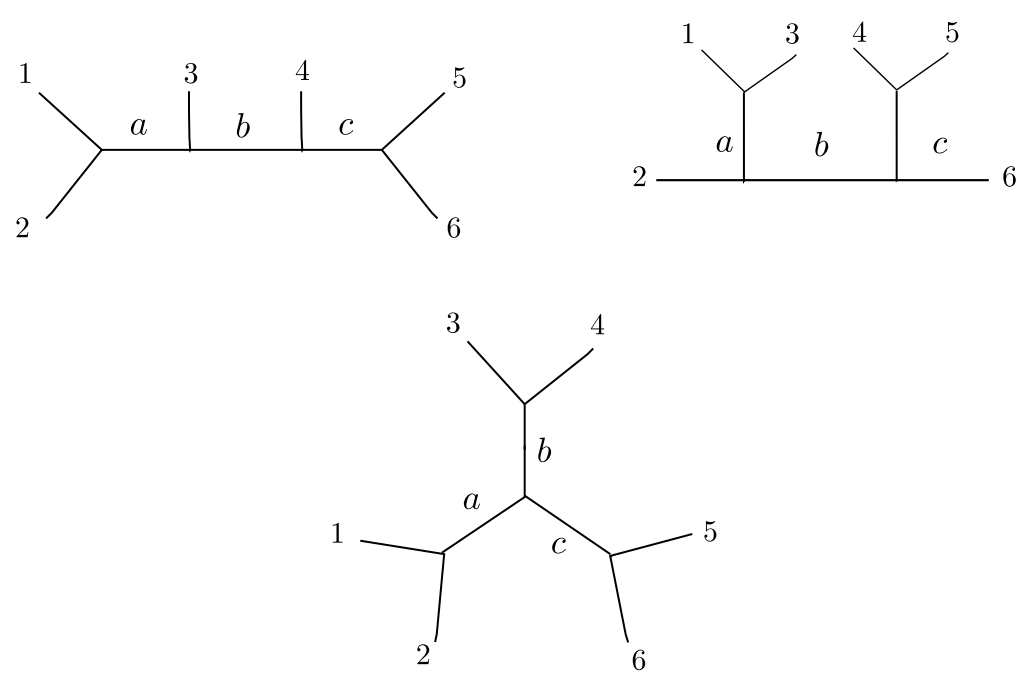

Figure 7. Three of the possible different ways to compute a 6 -point function by successive OPEs. Summation over the intermediate operators $a, b, c$ is implied.

the OPE. The different orderings can be encoded in trivalent tree diagrams with (singleparticle) operators on the external lines. For example let us consider a 6-point function $\left\langle\mathcal{O}_{1} \ldots \mathcal{O}_{6}\right\rangle$. Let us do the OPE in the following order $(1 \cdot 2) \rightarrow a,(a \cdot 3) \rightarrow b,(b \cdot 4) \rightarrow$ $c,(c \cdot 5) \rightarrow 6$. This is depicted in the first graph in figure 3 . In the same figure we also show two other possible orderings of doing the OPE.

Here it is convenient to introduce a generalization of the conformal partial waves for higher $n$-point functions $\left\langle\mathcal{O}_{1}\left(x_{1}\right) \ldots \mathcal{O}_{n}\left(x_{n}\right)\right\rangle$ i.e. to introduce special functions which encode parts of CFT correlators which depend on kinematics and not on the dynamics of any specific CFT. First we choose a given order of doing the successive OPEs to compute the $n$-point function. This ordering can be represented by a trivalent graph $\mathbf{T}$. The external legs of the graph are attached to the operators $\left\{\mathcal{O}_{i}\left(x_{i}\right)\right\}$ whose correlator we want to compute. Each of the internal legs is labeled by a conformal primary operator of given dimension and spin. At each vertex of the tree we perform the OPE and we keep only the operator indicated by the corresponding internal line of the graph together with all of its conformal descendants. This procedure gives a contribution to the $n$-point function which is equal to a certain product of OPE coefficients $C_{i j}^{k}$ times a special function of the external positions $x_{1}, \ldots, x_{n}$ which only depends on the choice of the tree $\mathbf{T}$ and the conformal dimensions and spins of the operators appearing on the legs of the tree. This special function $\mathbf{G}_{\left\{\mathcal{O}_{i}\right\},\{a, b, c \ldots\}}^{\mathbf{T}}\left(x_{1}, \ldots, x_{n}\right)$ is the $n$-point analogue of the conformal partial wave. Its functional dependence on the coordinates of the external legs is completely fixed by kinematics. While we do not have explicit expressions for these generalized CPWs we hope it is clear from their definition that in principle these functions do exist and they are indeed determined by kinematics of the conformal group.

If we sum over all possible intermediate operators (not necessarily single-particle ones) we reproduce the full $n$-point function. Hence the $n$-point function can be written as a 
sum of the form

$$
\left\langle\mathcal{O}_{1}\left(x_{1}\right) \ldots \mathcal{O}_{n}\left(x_{n}\right)\right\rangle=\sum_{a, b, c \ldots}[C C \ldots]^{\mathbf{T}} \mathbf{G}_{\left\{\mathcal{O}_{i}\right\},\{a, b, c \ldots\}}^{\mathbf{T}}\left(x_{1}, \ldots, x_{n}\right)
$$

where the generalized conformal partial waves $\mathbf{G}$ depend only on kinematics and the conformal dimensions and spins of the involved operators, and all the dynamics is contained in the 3-point function coefficients. The notation $[C C \ldots]^{\mathbf{T}}$ refers to a product of OPE coefficients for the operators $\mathcal{O}_{i}$ and $a, b, c \ldots$ where the way that the indices are contracted is dictated by the tree graph $\mathbf{T}$.

The conformal bootstrap conditions are based on the idea that we can do the OPE in any order we want and the resulting $n$-point function will be the same. In equations this means that we must have

$$
\sum_{a, b, c \ldots}[C C \ldots]^{\mathbf{T}_{\mathbf{i}}} \mathbf{G}_{\left\{\mathcal{O}_{i}\right\},\{a, b, c \ldots\}}^{\mathbf{T}_{\mathbf{i}}}\left(x_{1}, \ldots, x_{n}\right)=\sum_{a, b, c \ldots}[C C \ldots]^{\mathbf{T}_{\mathbf{j}}} \mathbf{G}_{\left\{\mathcal{O}_{i}\right\},\{a, b, c \ldots\}}^{\mathbf{T}_{\mathbf{j}}}\left(x_{1}, \ldots, x_{n}\right)
$$

for all possible trees $\mathbf{T}_{i}, \mathbf{T}_{j}$.

These equations are generalizations of the basic conformal bootstrap equations (3.6) for higher $n$-point functions. As we said, if we impose (3.6) for all possible operators on the external legs then the general condition (6.12) will be satisfied.

Conversely, in a generalized free CFT if we impose (6.12) for all $n$, but only for singleparticle operators on the external legs (and arbitrary operators on the internal legs), then the conformal bootstrap (3.6) will be automatically satisfied for all many-particle operators.

In the previous section we argued that the solution to the bootstrap equations for the 4-point function can be naturally reorganized in terms of tree-level Witten diagrams. This reorganization was natural because each Witten diagram solved the bootstrap conditions by itself. Now we want to sketch how the same reorganization might be performed for higher $n$-point functions of single-particle operators. In other words we want to consider the leading order $n$-point function and show how it can be naturally divided into certain parts, each of which solves the bootstrap conditions by itself. We want to argue that these parts coincide with tree-level Witten diagrams.

Let us consider the correlator $\left\langle\mathcal{O}_{1}\left(x_{1}\right) \ldots \mathcal{O}_{n}\left(x_{n}\right)\right\rangle$ and isolate from the sum (6.11) a term $\mathbf{T}$ which contains only single-particle operators in all the intermediate lines. We want

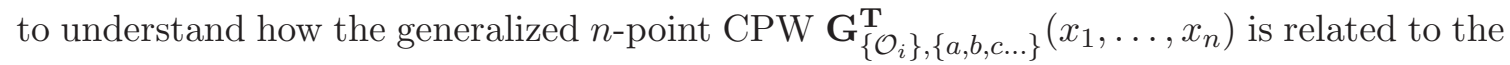
corresponding Witten diagram with trivalent vertices. It is not hard to see that the $n$-point $\mathrm{CPW}$ has nice factorization properties (i.e. consistent with an OPE and the scaling (6.7)) in the "direct channel" but not in the crossed channels.

From the discussion of the 4-point function in the previous subsection it is clear what we have to do to fix this problem. We add to the $n$-point CPW a series of $n$-point CPWs where in the intermediate channel we have multi-particle operators running, in such a way that the resulting "dressed" CPW has nice factorization properties in all channels. Presumably this can be done in a unique way if we restrict the spin of the intermediate operators and demand that the resulting amplitude has the correct singularities in the 
Lorentzian regime. We expect that the dressed $n$-point CPW thus constructed should coincide with the corresponding trivalent tree level Witten diagram.

In addition to these trivalent diagrams, we also have to include the contributions from quartic and higher interactions in order to reproduce the full $n$-point function, in analogy to what happened for the 4-point function.

\subsection{Generalized free CFTs and expansion in Witten diagrams}

To summarize: in a perturbed generalized free CFT, the solution to the bootstrap equations at "tree level" i.e. leading order in the $1 / c$ expansion, can be expressed as follows: to each single particle operator we assign a dual field $\phi_{i}$. For each 3-point function between single particle operators include a coupling $C_{i j k} \int d^{d+1} x \phi_{i} \phi_{j} \phi_{k}$. With this bulk Lagrangian we compute all exchange Witten diagrams in all possible channels. These should reproduce the part of the $n$-point functions with single particle operators running in any possible factorization channel. To reproduce the remaining part of the $n$-point function we add quartic and higher interactions terms (possibly with higher derivatives).

Formally any perturbed generalized free CFT can be reorganized in this way. In particular we have not assumed that the bulk theory corresponds to a large macroscopic AdS space. To see what this means let us consider for example the $\mathcal{N}=4 \mathrm{SYM}$ at large $N$ and small 't Hooft coupling where we know that the dual theory is IIB string theory in a highly curved AdS space. According to what we have discussed it is possible to reorganize the $1 / N$ expansion of this theory in terms of "Witten diagrams", i.e. in terms of dressed conformal blocks. On a basic level the $1 / \mathrm{N}$ expansion of the $\mathcal{N}=4 \mathrm{SYM}$ can be written in terms of Feynman diagrams. Since the theory remains conformal in the $1 / N$ expansion, the Feynman diagram expansion can be regrouped and organized into a conformal partial wave expansion. Finally, as we argued above, this conformal partial wave expansion can be further reorganized in terms of dressed conformal blocks (or "Witten diagrams"), since this basis is more natural for the $1 / N$ expansion of large $N$ gauge theories. The main difference between the "Witten diagram expansion" of the $\mathcal{N}=4$ at weak and strong 't Hooft coupling is that in the first case we have a very large number (exponentially growing with conformal dimension ${ }^{76}$ ) of intermediate single particle states, while in the second case we only have a small number corresponding to the chiral primaries of the CFT which survive at strong coupling.

However, our perspective is not to distinguish between CFTs which have classical gravity duals from those with "stringy" duals. For us the main distinction is to be made between theories whose low-lying spectrum has an effective description in terms of a finite number (with respect to the $c$-scaling) of degrees of freedom in AdS from those where such a dual description is not useful at all. The latter case would correspond to large $c$ CFTs with a low-lying spectrum whose degeneracy grows with $c$, as explained in section 2.3.

Before closing this section let us mention that in this section we have only discussed the conformal bootstrap of the low-lying sector of the CFT which consists of the (perturbed)

\footnotetext{
${ }^{76}$ but the number is $N$-independent and in that sense it is still a small number of exchanged operators in comparison to the $N^{2}$ fundamental fields of the Lagrangian.
} 
generalized free fields. In principle the bootstrap conditions have to be checked for all local operators of the CFT, even those whose conformal dimension is of order $c$ and which are not generalized free fields in any sense. In other words, even if we show that the correlators of low-lying operators satisfy the bootstrap conditions it is not a sufficient condition to guarantee the existence of such a CFT but only a necessary one.

\section{The CFT at finite temperature}

In this section we will discuss some issues about the finite temperature behavior of the theories under consideration.

\subsection{Factorization requires an infinite number of degrees of freedom}

As we argued in section 4 we can have factorization of correlators only if $c \rightarrow \infty$, where $c$ is defined by the 2-point function of the stress energy tensor. In a certain sense $c$ is sensitive to the number of fields in the theory i.e. the number of degrees of freedom. ${ }^{77}$ In this section we want to give some additional explanations of this statement and thus argue that a sector with factorized correlators can only emerge in a theory with an infinite number of degrees of freedom. ${ }^{78}$ The equivalent statement in the bulk is that gravity can be decoupled only if $G_{N} R_{\text {AdS }}^{1-d} \rightarrow 0$. In this limit the entropy of a black hole of finite size in AdS units diverges, as can be seen from the formula $S=\frac{A}{4 G_{N}}$, indicating a very large number of degrees of freedom.

A more intuitive way to count the number of degrees of freedom of a CFT is the following: consider the CFT on $\mathbf{R}^{d-1}$ and at temperature $T$. By scale invariance the entropy density must be of the form

$$
s \sim \widetilde{c} T^{d-1}
$$

with some convention-dependent constant of proportionality of order one. The constant $\widetilde{c}$ can be thought of as counting the degrees of freedom. In two dimensions Cardy's formula implies that $\widetilde{c}=c$ (in the appropriate conventions). In higher dimensional theories there is no such direct relation between $c$ and $\widetilde{c}$. Notice that if the CFT has a classical gravitational dual $^{79}$ then again it is true that $\widetilde{c}=c$, but more generally this relation does not have to be satisfied. For example in the $\mathcal{N}=4 \mathrm{SYM}$ the ratio $\widetilde{c} / c$ varies continuously between the value $4 / 3$ at weak 't Hooft coupling to the value 1 at strong coupling. This directly shows that there cannot be a simple general relation between the two constants in higher dimensional CFTs.

However, we would like to claim that if for a sequence of theories $c \rightarrow \infty$, then we must also have $\widetilde{c} \rightarrow \infty$. Ideally it would be useful if we could find some model-independent

\footnotetext{
${ }^{77}$ See appendix $\mathrm{C}$ for a discussion of the notion of central charge and the number of degrees of freedom in higher dimensional CFTs.

${ }^{78}$ Of course we are talking only about the nontrivial version of factorization, that is for operators which are not ordinary free fields i.e. for operators with $\Delta>\frac{d-2}{2}$. Obviously for operators with $\Delta=\frac{d-2}{2}$ we have factorization even for finite number of degrees of freedom, but this case is of no interest to us as these fields do not have a holographic description.

${ }^{79}$ I.e. a gravitational dual theory governed by the Einstein-Hilbert action.
} 
bounds for the ratio $\frac{\widetilde{c}}{c}$. Let us say that we could argue that independent of the CFT, there are two positive real numbers $\lambda_{1}, \lambda_{2}$ such that

$$
\lambda_{1} \leq \frac{\widetilde{c}}{c} \leq \lambda_{2}
$$

Then our claim would follow immediately. We have not been able to show such a statement. Notice however that if one considers the most general weakly coupled CFT with $n_{s}$ scalars, $n_{f}$ fermions and $n_{v}$ vectors, and one scans all possible values of $n_{s}, n_{f}, n_{v}$ one then finds that the ratio $\frac{\widetilde{c}}{c}$ is indeed bounded by two numbers $\lambda_{1}, \lambda_{2}$ [83], while for strongly coupled CFTs with gravity duals the ratio is equal to one. These facts are an indication that such a bound may actually exist.

In any case, without such a model-independent bound, our only hope is to find an indirect argument about the relation between $c$ and $\widetilde{c}$.

In two dimensional conformal field theories we have $c=\widetilde{c}$ due to the Cardy formula, which is usually proved by using modular invariance. As we explained in section 3.3 there is an alternative derivation of the two dimensional Cardy formula which may be more relevant in generalizing to higher dimensional CFTs: for any CFT in any dimension the following equation must hold

$$
\frac{\partial\left\langle T_{00}\right\rangle_{\beta}}{\partial \beta}=-\frac{1}{\beta} \int d^{d} x\left\langle T_{00}(x) T_{00}(0)\right\rangle_{\beta}^{c}
$$

where the integral is over $\mathbf{R}^{d-1} \times \mathbf{S}^{1}$ and the superscript $c$ in the 2-point function stands for "connected". ${ }^{80}$ The left hand side of equation (7.2) is related to the constant $\widetilde{c}$ by the following form of the thermal expectation value

$$
\left\langle T_{00}\right\rangle_{\beta} \sim \frac{\widetilde{c}}{\beta^{d}}
$$

which can be derived by basic thermodynamics from (7.1).

In two dimensions the thermal 2-point function $\langle T(z) T(0)\rangle_{\beta}$ on $\mathbf{R} \times \mathbf{S}^{1}$ can be computed exactly using the exponential map, or from holomorphy. Then using formula (3.12) we can derive $\widetilde{c}=c$. In higher dimensions we cannot compute the thermal 2-point function $\left\langle T_{00}(x) T_{00}(0)\right\rangle_{\beta}$ exactly. We can, however, still try to see how it scales with $c$. We consider the OPE of the stress tensor with itself. It has the following general form, suppressing Lorentz indices

$$
T(x) T(0)=\frac{c}{|x|^{2 d}}+\ldots+\frac{T(0)}{|x|^{d}}+\ldots
$$

Evaluating this at finite temperature we find schematically

$$
\left\langle T_{00}(x) T_{00}(0)\right\rangle_{\beta}=\frac{c}{|x|^{2 d}}+\ldots+\frac{\widetilde{c}}{\beta^{d}} \frac{1}{|x|^{d}}+\ldots
$$

Inserting into (3.12) we find an equation relating $c$ and $\widetilde{c}$, along with other 1-point functions. Unlike in $2-d$ we cannot use this relation to fix the ratio $\widetilde{c} / c$ because we need to know the

\footnotetext{
${ }^{80}$ I.e. $\left\langle T_{00}(x) T_{00}(0)\right\rangle_{\beta}^{c} \equiv\left\langle T_{00}(x) T_{00}(0)\right\rangle_{\beta}-\left\langle T_{00}(0)\right\rangle_{\beta}^{2}$.
} 
1-point functions of other operators which appear on the r.h.s. of (7.3). However unless certain nontrivial cancellations take place, we can see that the statement that $c \rightarrow \infty$ suggests that $\widetilde{c} \rightarrow \infty$. Even though it is definitely not a proof, this argument suggests that the central charge $c$ in the 2-point function of the stress tensor can go to infinity only if we have an infinite number of degrees of freedom (i.e. only if $\widetilde{c} \rightarrow \infty$ ).

\subsection{Hawking-Page transition?}

What happens to a generalized free CFT when we turn on a small temperature? As we explained, a generalized free CFT should be thought as a low-lying sector in a sequence of CFTs of increasing central charge $c$. In the limit $c \rightarrow \infty$ and at low conformal dimension (i.e. of order $c^{0}$ ) we have a finite number (i.e. their number does not scale with $c$ ) of generalized free fields, together with their multiparticle states. On the other hand at large enough conformal dimension the spectrum has to be modified and the entropy at high enough temperature should become $c$ dependent.

Intuitively, and judging from what happens in large $N$ gauge theories, one would expect that a generalized free CFT has two phases, one at low temperatures, which can be effectively described as a finite temperature Fock-space of generalized free field excitations and one at high temperatures where the generalized free fields are no longer a good description and where the underlying fundamental degrees of freedom are deconfined. One would expect the low temperature phase to have an entropy density which scales like $c^{0}$ while the high temperature phase has an entropy density of order $c$. More precisely, we have shown that so long as the GFF's free Fock space structure approximately holds, i.e. $\Delta \ll c$, the theory is "confined" as its entropy $S \sim T^{d}$ is $c$-independent. At sufficiently high temperatures, however, conformal invariance implies that this must give way to a "deconfined" holographic phase with extensive entropy $S \sim c T^{d-1}$, dominated by states with $\Delta \gg c$.

Moreover at $\Delta \sim c$ we expect the freely generated Fock space structure to be modified by interactions. Recasting these estimates ${ }^{81}$ using thermodynamic relations the confined phase has $S \sim \Delta^{\frac{d}{d+1}}$ while the deconfined phase is characterized by $S \sim c^{\frac{1}{d}} \Delta^{\frac{d-1}{d}}$. The crossover between these entropies occurs at $\Delta \sim c^{d+1}$, far beyond the regime of validity of the free Fock space approximation. The high temperature entropy is valid if $T=\left(\frac{\Delta}{c}\right)^{1 / d} \gg 1$ so it does not necessarily hold in the regime where the Fock space structure starts breaking down, $\Delta \sim c$. Nonetheless, even slightly beyond this regime (e.g. $\Delta \sim c^{1+\epsilon}$ ) the temperature is parametrically large (at large $c$ ) and the black hole entropy is significantly larger than the (uncorrected) Fock space entropy. In this regime,

$$
\frac{S_{\text {deconfined }}}{S_{\text {confined }}} \sim c^{\frac{1}{d+1}}
$$

suggesting that the transition from the confined to the deconfined phase is not likely smooth and thus has the character of a phase transition. In particular it is hard to imagine that corrections to the GFF spectrum are sufficient to account for this parametrically large entropy difference.

\footnotetext{
${ }^{81}$ Trading the temperature for the mean conformal dimension $\langle\Delta\rangle$ in both the confined and the deconfined ensemble. This is of course only valid in the regimes $\Delta \ll c$ and $\Delta \gg c$, respectively.
} 
Thus quite generally we expect that the two phases are separated by a phase transition. Bulk intuition would suggest that this occurs at some finite temperature of order one as it does in the Hawking-Page phase transition [30, 31, 105]. This is indeed what happens in weakly coupled large $N$ gauge theories with fields in the adjoint of the gauge group and in large $N$ symmetric orbifold CFTs in two dimensions as we reviewed in section 2.2.

However there are indications that the story may be more complicated and the phase structure of generalized free CFTs may involve additional possibilities than those which are realized in large $N$ gauge theories. In particular it seems that there are examples of generalized free CFTs where the analogue of the Hawking-Page phase transition does not take place at temperatures of order 1 but at a temperature which goes to infinity as we take $c \rightarrow \infty$. This suggests the peculiar result that there are no standard black holes in the bulk (i.e. whose temperature is of the order of the AdS scale).

\subsection{Extending the "Cardy regime"?}

From the discussion in the last subsection it is clear that in the "generalized Cardy regime", $\underset{\tilde{c}}{\Delta} \gg 1$, the dual CFT exhibits black hole-like entropy suggesting the bulk is dominated, in this regime, by a large AdS black hole (which can be stringy in nature [31, 106]). On the other hand, arguments from general relativity [34] imply that the transition to a black hole dominated phase occurs at a temperature of order one (in AdS units). From the relation between $c$ and $\tilde{c}$, however, it follows that the generalized Cardy regime corresponds to a much higher temperature $T \sim\left(\frac{\Delta}{c}\right)^{\frac{1}{d}} \gg 1$. Thus CFTs which admit Einstein-Hilbert gravity duals ${ }^{82}$ require that the generalized Cardy formula discussed in the previous section to hold already at $T \sim \mathcal{O}(1)$.

In the black hole phase $S \sim c T^{d-1}$ and $\Delta \sim c T^{d}$ so if this is to hold already at $T \sim \mathcal{O}(1)$ then the CFT must have $e^{c}$ states with $\Delta \sim c$. This extended Cardy regime is, in fact, evident in the examples discussed in section 2.2. For instance, in $\mathcal{N}=4$, the entropy $S \sim N^{1 / 2} E^{3 / 4}$ can be derived from general CFT considerations at high-temperature, $T \sim$ $\left(E / N^{2}\right)^{1 / d} \gg 1$. In the free theory, however, it is not hard to see that already at $E \sim N^{2}$ we have $S \sim N^{2}$ so the entropy formula seems to apply already at $T \sim \mathcal{O}(1)$. From the growth $S \sim N^{2}$ at $E \sim N^{2}$ we see that, in the canonical ensemble, the phase transition alluded to above occurs at $T \sim \mathcal{O}(1)$ because the free energy goes as $F \sim \alpha N^{2}-\gamma N^{2} T$ with $\alpha, \gamma$ order one numbers. In symmetric orbifold theories the existence of twist sectors with twist $k$ and a mass gap $1 / k$ results in an "effective temperature" in these sectors that grows with $k$ even while the real temperature (measured in e.g. units of the CFT circle) is order one.

While $\mathcal{N}=4 \mathrm{SYM}$ and symmetric orbifolds seem to exhibit this "extended Cardy regime" there is no reason for it to hold more generally. On the other hand $T \sim 1$ is well-outside the Cardy regime in 2-d CFTs yet, in many known examples, Cardy's formula seems to hold in this regime nonetheless. In particular this seems to hold for free bosons in two-dimensional theories which provide a rather simple example.

\footnotetext{
${ }^{82}$ Recall our general approach incorporates bulk duals that may include many other degrees of freedom beyond pure GR - e.g. bulks that are highly curved in "string units".
} 


\section{Discussions}

We have tried to highlight the basic features of conformal field theories with holographic duals. We emphasized the importance of operators whose correlators factorize as the basic ingredient for the emergence of the bulk. We also explained that such operators with factorized correlators can only be realized in a limiting sense as a small low-lying sector of very big conformal field theories.

In our paper we have not addressed an important point. We argued that the interactions between generalized free fields can be related to effective bulk interactions between fields in AdS but we have not discussed whether the bulk fields and their interactions admit an independent (dual) formulation. For instance, in the case of large $N$ gauge theories we generally expect that there is a dual two-dimensional worldsheet CFT which can reproduce the interactions between spacetime fields by the usual perturbative string-theory rules. Is there such a dual theory for more general generalized free CFTs? Or should generic dual theories that emerge from this construction be thought of as entirely "effective", lacking an underlying organizing principle. The problem with the latter viewpoint is that in general we expect that the number of fields in the AdS space will be quite large (though $c$-independent), similar to what happens in the $\mathcal{N}=4$ SYM at weak 't Hooft coupling or in the $O(N)$ vector models. ${ }^{83}$ In these cases the bulk theory is not a conventional effective field theory with a small number of fields so without an organizing principle (such as that provided by perturbative string theory) it is not so clear how to handle the bulk theory. On the other hand the answer cannot be that there is always a dual string theory, as can be seen from the example of M-theory backgrounds (for example, $\mathrm{AdS}_{4} \times \mathrm{S}^{7}$ or $\mathrm{AdS}_{7} \times \mathrm{S}^{4}$ ) which presumably have no description in terms of a perturbative string theory, or from examples of higher-spin gravity.

Our paper focused on the holographic representation of conformal field theories. However, conformal invariance does not play a fundamental role in our discussions; it was merely a simplifying assumption. More generally let us consider a non-conformal QFT with a local operator $\mathcal{O}$ whose correlators factorize to products of 2-points functions (in an appropriate limiting sense). As we argued in the text it would be natural to represent this field in a higher dimensional space, whose geometry has to be chosen so that the field will satisfy a linear wave equation on that background and that the bulk propagator will coincide with the boundary 2-point function.

An interesting problem would be to examine a generalized free CFT whose low-lying spectrum consists only of the stress energy tensor and multiparticle states. If such a CFT existed it would be dual to pure gravity in AdS. It would be interesting to study to what extent the conformal bootstrap can constrain the tree-level (i.e. leading order in an $1 / c$ expansion) interactions between the stress energy tensor to be the same as those predicted by tree level Einstein-Hilbert gravity in the bulk. ${ }^{84}$ Unfortunately this problem is difficult

\footnotetext{
${ }^{83}$ In other words, typically we do not expect a generalized free CFT to have a gap between single trace operators of spin two and higher as in [10], which would lead to a dual with a large macroscopic AdS spacetime.

${ }^{84}$ In other words, to what extent AdS theories with higher-derivative corrections are inconsistent if the
} 
in practice since the conformal partial waves for operators with nonzero spin on the external legs are not explicitly known. Along these lines, it might be interesting to explore the use of analyticity methods, in the spirit of the $\mathrm{BCFW}$ relations and their generalizations for AdS [111], to simplify the bootstrap problem for correlators of the stress energy tensor.

A related, but perhaps simpler, problem would be to consider the simplest perturbed GFF we can construct. This would include a single GFF scalar $\mathcal{O}$ which factorizes to order $c^{0}$ and which at order $\frac{1}{\sqrt{c}}$ receives only corrections mediated by the stress tensor. Thus one could attempt to construct a consistent CPW expansion by relating a graviton exchange diagram to a CPW expansion. This would presumably include stress-tensor and perhaps some multi-particle contributions. Despite requiring higher-spin intermediate operators this approach depends only on the CPW expansion of scalar four-point functions and is hence accessible with current technology. In the bulk this should correspond to a free scalar minimally coupled to gravity.

In this paper we focused on the leading terms in the large $c$ expansion and their representation in terms of tree-level interactions in the bulk. It would be interesting to further study the subleading terms corresponding to loop diagrams. This was also recently explored in [13].

It would be satisfying to find examples of CFTs with generalized free sectors which are not large $N$ gauge theories (or symmetric orbifolds in two dimensions). This would clarify the fact that the holographic correspondence is not intimately related to gauge invariance but rather to the fact that generalized free fields can be realized linearly in a higher dimensional AdS space.

Finally a natural question is the following: we saw that free (or weakly interacting) fields in AdS can be thought of as a representation of generalized free fields of a CFT which makes the linearity more manifest. What is the analogue for flat space/de Sitter fields? What is the class of quantum systems for which free fields in flat space/de Sitter provide an effective (approximately) linearized description via a holographic map?

\section{Acknowledgments}

We would like to thank R. Brustein, F. Dolan, M. Gaberdiel, R. Gopakumar, N. Iizuka, E. Kiritsis, M. Kulaxizi, S. Minwalla, A. Petkou, N. Prezas, S. Raju, S. Rychkov, M. Shigemori for useful discussions. We would like to especially thank J. de Boer and E. Verlinde for useful comments and stimulating discussions. We would also like to thank J. de Boer, R. Gopakumar, E. Kiritsis for very helpful comments on the draft. K.P. would like to thank the University of Crete, the University of Amsterdam, CERN and CEA Saclay for hospitality during the completion of this work. S.E. would like to thank the University of Amsterdam and the CEA Saclay for hospitality during the completion of this work. The research of S.E. is partially supported by the Netherlands Organisation for Scientific Research (NWO) under a Rubicon grant.

only light particle in the spectrum is the graviton. For such an analysis it might be important to also analyze the consistency of the theory at finite temperature, as was partially done in [107-110]. We thank J. de Boer for discussions on these issues. 


\section{A Conformal partial waves}

In this appendix we review some of the results of [66] which are necessary for our discussions. We focus on four dimensional conformal field theories. We consider a 4-point function of scalar operators $\mathcal{O}_{i}, i=1,2,3,4$ and an operator $\mathcal{O}_{k}$ of spin $l$ which is exchanged in the $(12) \rightarrow(34)$ channel. Then the conformal partial wave corresponding to $\mathcal{O}_{k}$ and all of its descendants is

$$
\mathbf{G}_{k}^{12,34}\left(x_{1}, x_{2}, x_{3}, x_{4}\right)=\frac{1}{\left|x_{12}\right|^{\Delta_{1}+\Delta_{2}}\left|x_{34}\right|^{\Delta_{3}+\Delta_{4}}}\left(\frac{x_{24}}{x_{14}}\right)^{\Delta_{12}}\left(\frac{x_{14}}{x_{13}}\right)^{\Delta_{34}} \bar{G}_{k}^{12,34}(u, v)
$$

where we introduced the conformal cross-ratios

$$
u=\frac{x_{12}^{2} x_{34}^{2}}{x_{13}^{2} x_{24}^{2}} \quad, \quad v=\frac{x_{14}^{2} x_{23}^{2}}{x_{13}^{2} x_{24}^{2}}
$$

which can also be parametrized as

$$
u=z \bar{z} \quad, \quad v=(1-z)(1-\bar{z})
$$

If we work in Euclidean signature then we have $\bar{z}=z^{*}$. The functions $\bar{G}_{k}^{12,34}(u, v)$ written in terms of the variables $z, \bar{z}$ have the form

$$
\begin{aligned}
\bar{G}_{k}^{12,34}(u, v)= & \left(-\frac{1}{2}\right)^{l} \frac{(z \bar{z})^{\frac{1}{2}\left(\Delta_{k}-l\right)}}{z-\bar{z}}\left[z^{l+1}{ }_{2} F_{1}\left(\frac{\Delta_{k}-\Delta_{12}+l}{2}, \frac{\Delta_{k}+\Delta_{34}+l}{2}, \Delta_{k}+l, z\right) \times\right. \\
& \left.\times{ }_{2} F_{1}\left(\frac{\Delta_{k}-\Delta_{12}-l-2}{2}, \frac{\Delta_{k}+\Delta_{34}-l-2}{2}, \Delta_{k}+l-2, \bar{z}\right)-(z \leftrightarrow \bar{z})\right]
\end{aligned}
$$

A 4-point function of scalar operators can be expanded in CPWs in the $(12) \rightarrow(34)$ channel

$$
\left\langle\mathcal{O}_{1}\left(x_{1}\right) \mathcal{O}_{2}\left(x_{2}\right) \mathcal{O}_{3}\left(x_{3}\right) \mathcal{O}_{4}\left(x_{4}\right)\right\rangle=\sum_{k} C_{12}^{k} C_{34}^{k} \mathbf{G}_{k}^{12,34}\left(x_{1}, x_{2}, x_{3}, x_{4}\right)
$$

This expansion converges when $x_{1}$ and $x_{2}$ can be enclosed by a sphere which does not contain $x_{3}$ or $x_{4}$, in other words when the conformal cross ratios are within a certain distance around the point $u=0, v=1$. In terms of the variables $z, \bar{z}$ the region of convergence of this expansion corresponds to the disk $|z|<1$.

In the other limit, where $x_{1}$ and $x_{4}$ can be enclosed by a sphere not containing the other insertions, we can expand in the $(14) \rightarrow(23)$ channel as

$$
\left\langle\mathcal{O}_{1}\left(x_{1}\right) \mathcal{O}_{2}\left(x_{2}\right) \mathcal{O}_{3}\left(x_{3}\right) \mathcal{O}_{4}\left(x_{4}\right)\right\rangle=\sum_{k} C_{14}^{k} C_{23}^{k} \mathbf{G}_{k}^{14,23}\left(x_{1}, x_{4}, x_{2}, x_{3}\right)
$$

which converges around the point $u=1, v=0$, or the disk $|1-z|<1$ on the $z$-plane.

The statement of the conformal bootstrap is that the two expansions are equivalent, in the sense that we have

$$
\sum_{k} C_{12}^{k} C_{34}^{k} \mathbf{G}_{k}^{12,34}\left(x_{1}, x_{2}, x_{3}, x_{4}\right)=\sum_{k} C_{14}^{k} C_{23}^{k} \mathbf{G}_{k}^{14,23}\left(x_{1}, x_{4}, x_{2}, x_{3}\right)
$$


in the overlapping region of convergence i.e. the region satisfying $|z|<1$ and $|1-z|<1$ simultaneously. This condition can also be written as

$$
\sum_{k} C_{12}^{k} C_{34}^{k} \bar{G}_{k}^{12,34}(u, v)=\sum_{k} C_{14}^{k} C_{23}^{k} \frac{u^{\left(\Delta_{3}+\Delta_{4}\right) / 2}}{v^{\left(\Delta_{2}+\Delta_{3}\right) / 2}} \bar{G}_{k}^{14,23}(v, u)
$$

\section{B CPW expansion of Witten diagrams}

In this appendix we present the CPW expansion of some basic Witten diagrams. These are not new results, but to our knowledge explicit expressions for the CPW coefficients in the case of operators with different conformal dimensions (i.e. equations (B.3), (B.8), (B.10)) have not been presented in the literature.

\section{B.1 Contact Witten diagram}

We only discuss the case of $\mathrm{AdS}_{5}$ i.e. we take $d=4$. The conformal dimension $\Delta$ is related to the mass of a field in the bulk by the formula ${ }^{85} \Delta=2+\sqrt{4+m^{2}}$. The AdS metric is

$$
d s^{2}=\frac{d z_{0}^{2}+d \vec{z}^{2}}{z_{0}^{2}}
$$

The bulk-to-boundary propagator has the form

$$
K_{\Delta}(z, x)=\frac{\Gamma(\Delta)}{\pi^{2} \Gamma(\Delta-2)}\left(\frac{z_{0}}{z_{0}^{2}+(\vec{z}-\vec{x})^{2}}\right)^{\Delta}
$$

and the bulk-to-bulk propagator

$$
G_{\Delta}(z, w)=\frac{\Gamma(\Delta)}{2^{\Delta+1} \pi^{2} \Gamma(\Delta-1)} s^{\Delta}{ }_{2} F_{1}\left(\frac{\Delta}{2}, \frac{\Delta+1}{2} ; \Delta-1, s^{2}\right)
$$

where we introduced the geodesic distance in AdS

$$
s=\frac{2 z_{0} w_{0}}{z_{0}^{2}+w_{0}^{2}+(\vec{z}-\vec{w})^{2}}
$$

Let us call $\mathbf{W}^{1234}\left(x_{1}, x_{2}, x_{3}, x_{4}\right)$ the basic contact Witten diagram for 4 scalar operators $\mathcal{O}_{i}$ of conformal dimension $\Delta_{i}$.

$$
\mathbf{W}^{1234}\left(x_{1}, x_{2}, x_{3}, x_{4}\right)=\int \frac{d^{5} z}{z_{0}^{5}} K_{\Delta_{1}}\left(z, x_{1}\right) K_{\Delta_{2}}\left(z, x_{2}\right) K_{\Delta_{3}}\left(z, x_{3}\right) K_{\Delta_{4}}\left(z, x_{4}\right)
$$

For simplicity we take the conformal dimensions to be generic real numbers. ${ }^{86}$ By massaging the expression for this Witten diagram we find that it can be decomposed in conformal blocks in the (12) $\rightarrow$ (34) channel as

$$
\begin{aligned}
\mathbf{W}^{1234}\left(x_{1}, x_{2}, x_{3}, x_{4}\right)= & \sum_{n=0}^{\infty} a_{n}\left(\Delta_{1}, \Delta_{2}, \Delta_{3}, \Delta_{4}\right) \mathbf{G}_{\Delta_{1}+\Delta_{2}+2 n}^{12,34}\left(x_{1}, x_{2}, x_{3}, x_{4}\right) \\
& +\sum_{n=0}^{\infty} a_{n}\left(\Delta_{3}, \Delta_{4}, \Delta_{1}, \Delta_{2}\right) \mathbf{G}_{\Delta_{3}+\Delta_{4}+2 n}^{12,34}\left(x_{1}, x_{2}, x_{3}, x_{4}\right)
\end{aligned}
$$

\footnotetext{
${ }^{85} \mathrm{We}$ assume that $m^{2}$ is large enough so that there is no ambiguity in the choice of the branch of the square root.

${ }^{86}$ In order to avoid logarithmic terms due to anomalous dimensions of two-particle operators.
} 
where the coefficients $a_{n}$ are given by

$$
\begin{aligned}
a_{n}\left(\Delta_{1}, \Delta_{2}, \Delta_{3}, \Delta_{4}\right)= & \frac{(-1)^{n}}{\pi^{6}} \frac{\Gamma\left(\Delta_{1}+n\right) \Gamma\left(\Delta_{2}+n\right) \Gamma\left(\Delta_{1}+\Delta_{2}+n-2\right)}{2 \Gamma\left(\Delta_{1}-2\right) \Gamma\left(\Delta_{2}-2\right) \Gamma\left(\Delta_{3}-2\right) \Gamma\left(\Delta_{4}-2\right)} \times \\
& \times \frac{\Gamma\left(\Sigma-\Delta_{3}+n\right) \Gamma\left(\Sigma-\Delta_{4}+n\right) \Gamma(\Sigma+n-2) \Gamma\left(\Sigma-\Delta_{1}-\Delta_{2}-n\right)}{\Gamma(n+1) \Gamma\left(\Delta_{1}+\Delta_{2}+2 n\right) \Gamma\left(\Delta_{1}+\Delta_{2}+2 n-2\right)}
\end{aligned}
$$

where $\Sigma=\frac{1}{2}\left(\Delta_{1}+\Delta_{2}+\Delta_{3}+\Delta_{4}\right)$. Our assumption that the conformal dimensions are generic means that none of the Gamma functions will blow up for any $n$.

The physical interpretation of this expansion is that in the $(12) \rightarrow(34)$ channel, the contact Witten diagram corresponds to the exchange of 2-particle conformal primaries : $\mathcal{O}_{1}\left(\nabla^{2}\right)^{n} \mathcal{O}_{2}:$ and $: \mathcal{O}_{3}\left(\nabla^{2}\right)^{n} \mathcal{O}_{4}:$ for $n=0,1, \ldots$, together with their descendants as predicted by conformal invariance.

The same diagram can be expanded in the crossed channel as

$$
\begin{aligned}
\mathbf{W}^{1234}\left(x_{1}, x_{2}, x_{3}, x_{4}\right)= & \sum_{n=0}^{\infty} a_{n}\left(\Delta_{1}, \Delta_{4}, \Delta_{2}, \Delta_{2}\right) \mathbf{G}_{\Delta_{1}+\Delta_{4}+2 n}^{14,23}\left(x_{1}, x_{4}, x_{2}, x_{3}\right) \\
& +\sum_{n=0}^{\infty} a_{n}\left(\Delta_{2}, \Delta_{3}, \Delta_{1}, \Delta_{4}\right) \mathbf{G}_{\Delta_{2}+\Delta_{3}+2 n}^{14,23}\left(x_{1}, x_{4}, x_{2}, x_{2}\right)
\end{aligned}
$$

where now we have the exchange of operators : $\mathcal{O}_{1}\left(\nabla^{2}\right)^{n} \mathcal{O}_{4}:$ and $: \mathcal{O}_{2}\left(\nabla^{2}\right)^{n} \mathcal{O}_{3}$ :. So we can explicitly see (though perhaps not surprisingly) that the basic contact diagram is consistent with an OPE expansion in all channels ${ }^{87}$ and is a solution of the bootstrap equations.

As a test that we got the expansion (B.3) right, let us check the bootstrap condition (A.3) numerically by summing up the conformal blocks with coefficients given by (B.2), (B.3) , (B.4) in the direct and crossed channels. To do this in practice, we regularize the infinite sums by taking $n$ up to an integer $N_{\max }$. In the following graph we plot the ratio $\frac{\text { direct }}{\text { crossed }}\left(N_{\max }\right)$ evaluated along the real axis $z=\bar{z}$ (see appendix A for the meaning of the variables $z, \bar{z}$ ), for increasing values of $N_{\max }$. In the range of the $z$-axis which is plotted, both expansions are convergent so the ratio should approach the value 1 for large $N_{\max }$. We see that this is indeed the case.

Let us see what this CPW expansion bodes for the OPE. By construction

$$
\lambda a_{n}\left(\Delta_{1}, \Delta_{2}, \Delta_{3}, \Delta_{4}\right)=C_{12}^{: 12 n:} C_{34}^{: 12 n:}\left\langle\mathcal{O}_{n}^{(12)} \mathcal{O}_{n}^{(12)}\right\rangle
$$

in a hopefully obvious notation. Here $\lambda$ is the coefficient of the bulk 4-pt vertex (which we had previously set to 1 ). As a consequence of the freedom allowed by $\lambda$ the real physical content of the expression $a_{n}$ is not in its value but in the ratio of $a_{n}$ with different arguments (i.e. $\left.a_{n}\left(\Delta_{1}, \Delta_{2}, \Delta_{3}, \Delta_{4}\right) / a_{n}\left(\Delta_{3}, \Delta_{4}, \Delta_{1}, \Delta_{2}\right)\right)$.

\footnotetext{
${ }^{87}$ Notice that in older literature the statement has appeared that a single Witten diagram is not fully consistent with an OPE expansion. However as we can see from the explicit expansion presented above this is not true. A single Witten diagram is consistent with an OPE expansion by itself. We believe that the reason for this confusion is that the 2-particle conformal primary states of the form : $\mathcal{O}_{1}\left(\nabla^{2}\right)^{n} \mathcal{O}_{2}$ : etc. were not properly taken into account in earlier works.
} 


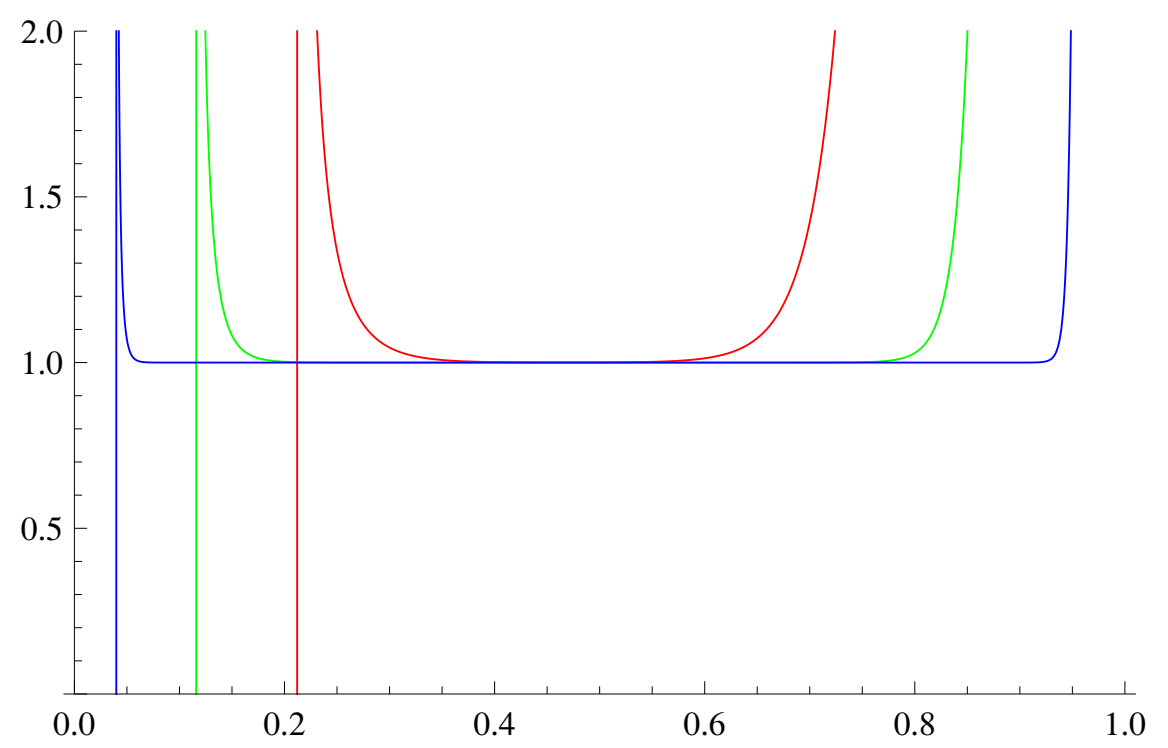

Figure 8. Plot of the ratio $\frac{\text { direct }}{\text { crossed }}\left(N_{\max }\right)$ as a function of $z=\bar{z}$ for $N_{\max }=5$ (red), $N_{\max }=10$ (green) and $N_{\max }=25$ (blue). We see that the ratio converges to 1 as we increase $N_{\max }$.

Because we know $C_{12}^{: 12 n}$ : and $\left\langle\mathcal{O}_{n}^{(12)} \mathcal{O}_{n}^{(12)}\right\rangle$ to first order (from factorization) we can

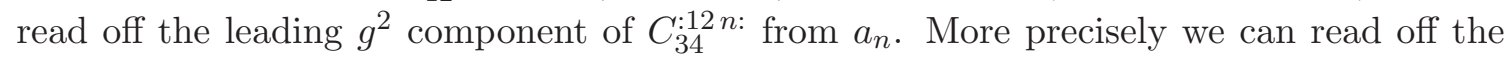

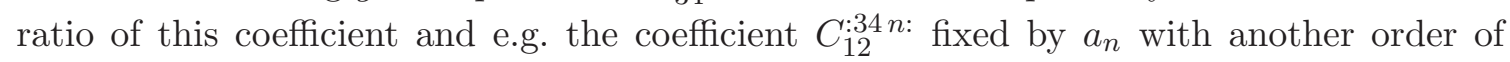
the arguments.

\section{B.2 Exchange Witten diagram}

Let us now consider the basic exchange Witten diagram $\mathbf{W}_{k}^{12,34}\left(x_{1}, x_{2}, x_{3}, x_{4}\right)$ with operators $\mathcal{O}_{i}, i=1, \ldots, 4$ of dimensions $\Delta_{i}$ on the external legs and the operator $\mathcal{O}_{k}$ of dimension $\Delta_{k}$ dual to the field exchanged between (12) and (34). We have

$$
\mathbf{W}_{k}^{12,34}\left(x_{1}, x_{2}, x_{3}, x_{4}\right)=\int \frac{d^{5} z}{z_{0}^{5}} \int \frac{d^{5} w}{w_{0}^{5}} K_{\Delta_{1}}\left(z, x_{1}\right) K_{\Delta_{2}}\left(z, x_{2}\right) G_{\Delta_{k}}(z, w) K_{\Delta_{3}}\left(w, x_{3}\right) K_{\Delta_{4}}\left(w, x_{4}\right)
$$

Let us first present its expansion in conformal partial waves in the direct (i.e. (12) $\rightarrow$ (34) channel). We have

$$
\begin{aligned}
\mathbf{W}_{k}^{12,34}\left(x_{1}, x_{2}, x_{3}, x_{4}\right)= & b\left(\Delta_{1}, \Delta_{2}, \Delta_{3}, \Delta_{4}, \Delta_{k}\right) \mathbf{G}_{k}^{12,34}\left(x_{1}, x_{2}, x_{3}, x_{4}\right) \\
& +\sum_{n=0}^{\infty} c_{n}\left(\Delta_{1}, \Delta_{2}, \Delta_{3}, \Delta_{4}, \Delta_{k}\right) \mathbf{G}_{\Delta_{1}+\Delta_{2}+2 n}^{12,34}\left(x_{1}, x_{2}, x_{3}, x_{4}\right) \\
& +\sum_{n=0}^{\infty} c_{n}\left(\Delta_{3}, \Delta_{4}, \Delta_{1}, \Delta_{2}, \Delta_{k}\right) \mathbf{G}_{\Delta_{3}+\Delta_{4}+2 n}^{12,34}\left(x_{1}, x_{2}, x_{3}, x_{4}\right)
\end{aligned}
$$


with the following coefficients

$$
\begin{array}{r}
b\left(\Delta_{1}, \Delta_{2}, \Delta_{3}, \Delta_{4}, \Delta_{k}\right) \\
=\frac{\Gamma\left(\frac{\Delta_{1}+\Delta_{2}-\Delta_{k}}{2}\right) \Gamma\left(\frac{\Delta_{3}+\Delta_{4}-\Delta_{k}}{2}\right) \Gamma\left(\frac{\Delta_{1}+\Delta_{2}+\Delta_{k}}{2}-2\right) \Gamma\left(\frac{\Delta_{3}+\Delta_{4}+\Delta_{k}}{2}-2\right)}{8 \pi^{6} \Gamma\left(\Delta_{1}-2\right) \Gamma\left(\Delta_{2}-2\right) \Gamma\left(\Delta_{3}-2\right) \Gamma\left(\Delta_{4}-2\right)} \times \\
\quad \times \frac{\Gamma\left(\frac{\Delta_{k}+\Delta_{1}-\Delta_{2}}{2}\right) \Gamma\left(\frac{\Delta_{k}-\Delta_{1}+\Delta_{2}}{2}\right) \Gamma\left(\frac{\Delta_{k}+\Delta_{3}-\Delta_{4}}{2}\right) \Gamma\left(\frac{\Delta_{k}-\Delta_{3}+\Delta_{4}}{2}\right)}{\Gamma\left(\Delta_{k}\right) \Gamma\left(\Delta_{k}-1\right)}
\end{array}
$$

and

$$
\begin{aligned}
& c_{n}\left(\Delta_{1}, \Delta_{2}, \Delta_{3}, \Delta_{4}, \Delta_{k}\right) \\
& =\frac{(-1)^{n+1}}{\pi^{6}} \frac{\Gamma\left(\Delta_{1}+n\right) \Gamma\left(\Delta_{2}+n\right) \Gamma\left(\Delta_{1}+\Delta_{2}+n-2\right)}{2 \Gamma\left(\Delta_{1}-2\right) \Gamma\left(\Delta_{2}-2\right) \Gamma\left(\Delta_{3}-2\right) \Gamma\left(\Delta_{4}-2\right)} \times \\
& \quad \times \frac{\Gamma\left(\Sigma-\Delta_{3}+n\right) \Gamma\left(\Sigma-\Delta_{4}+n\right) \Gamma(\Sigma+n-2) \Gamma\left(\Sigma-\Delta_{1}-\Delta_{2}-n\right)}{\Gamma(n+1) \Gamma\left(\Delta_{1}+\Delta_{2}+2 n\right) \Gamma\left(\Delta_{1}+\Delta_{2}+2 n-2\right)\left(\Delta_{1}+\Delta_{2}-\Delta+2 n\right)\left(\Delta_{1}+\Delta_{2}+\Delta-4+2 n\right)}
\end{aligned}
$$

In the direct channel (i.e. $(12) \rightarrow(34)$ ) the exchange Witten diagram corresponds to the exchange of the conformal block of the operator $\mathcal{O}_{k}$, together with the exchange of the conformal blocks of 2-particle operators of the form : $\mathcal{O}_{1}\left(\nabla^{2}\right)^{n} \mathcal{O}_{2}:$ and : $\mathcal{O}_{3}\left(\nabla^{2}\right)^{n} \mathcal{O}_{4}:$.

Note that once more we can relate $b_{n}$ and $c_{n}$ to a product of OPE coefficients. $b_{n}$ is proportional to the product of two unknown (order $g$ ) coefficients $C_{12}^{k} C_{34}^{k}$ but from its structure there appears to be a natural factorization into two components depending only on $\left(\Delta_{1}, \Delta_{2}\right)$ and $\left(\Delta_{3}, \Delta_{4}\right)$. Of course, as in the case with $a_{n}$, the expansion is only fixed up to a coefficient (in this case the product of two three-point vertices $g_{12 k} g_{34 k}$ in the bulk) so the physical content is actually in the ratio of the $b_{n}$ to the $c_{n}$. This translates into a

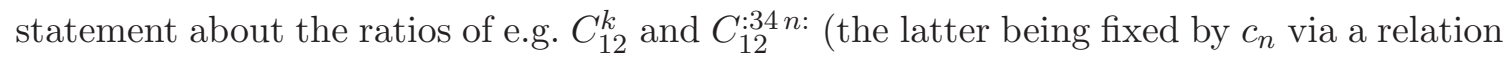
analogous to (B.5)).

Expanding the Witten diagram in the crossed channel is more complicated. Starting with the expressions of [91] and following the method of [97, 98] we find that the same exchange Witten can be expanded in the crossed channel (i.e. $(14) \rightarrow(23)$ ) as

$$
\begin{aligned}
\mathbf{W}_{k}^{12,34}\left(x_{1}, x_{2}, x_{3}, x_{4}\right)= & \sum_{n=0}^{\infty} d_{n}\left(\Delta_{1}, \Delta_{4}, \Delta_{2}, \Delta_{3}, \Delta_{k}\right) \mathbf{G}_{\Delta_{1}+\Delta_{4}+2 n}^{14,23}\left(x_{1}, x_{4}, x_{2}, x_{2}\right) \\
& +\sum_{n=0}^{\infty} d_{n}\left(\Delta_{2}, \Delta_{3}, \Delta_{1}, \Delta_{4}, \Delta_{k}\right) \mathbf{G}_{\Delta_{2}+\Delta_{3}+2 n}^{14,23}\left(x_{1}, x_{4}, x_{2}, x_{3}\right)
\end{aligned}
$$

which corresponds to the exchange of two-particle operators : $\mathcal{O}_{1}\left(\nabla^{2}\right)^{n} \mathcal{O}_{4}$ : and : $\mathcal{O}_{2}\left(\nabla^{2}\right)^{n} \mathcal{O}_{3}$ :. We have not been able to find an explicit closed form for the coefficients $d_{n}$ but they can be determined by solving the following equations

$$
\sum_{n=0}^{\infty} d_{n}\left(\Delta_{1}, \Delta_{4}, \Delta_{2}, \Delta_{3}, \Delta_{k}\right) \bar{G}_{\Delta_{1}+\Delta_{4}+2 n}(u, v)=h u^{\frac{\Delta_{1}+\Delta_{4}}{2}} \sum_{n, m=0}^{\infty} I_{m n} \frac{u^{m}(1-v)^{n}}{m ! n !}
$$


where $\bar{G}(u, v)$ are related to the CPWs by equation (A.1) and

$$
h=\frac{\Gamma\left(\frac{\Delta_{1}+\Delta_{2}+\Delta_{3}+\Delta_{4}-4}{2}\right) \Gamma\left(\frac{\Delta_{1}+\Delta_{2}+\Delta_{k}-4}{2}\right) \Gamma\left(\frac{\Delta_{3}+\Delta_{4}+\Delta_{k}-4}{2}\right) \Gamma\left(\frac{\Delta_{3}-\Delta_{4}-\Delta_{1}+\Delta_{2}}{2}\right)}{8 \pi^{6} \Gamma\left(\Delta_{1}-2\right) \Gamma\left(\Delta_{2}-2\right) \Gamma\left(\Delta_{3}-2\right) \Gamma\left(\Delta_{4}-2\right) \Gamma\left(\Delta_{k}-1\right)}
$$

The coefficients $I_{m n}$ are given by the following integral

$$
\begin{aligned}
I_{m n}= & \frac{1}{2 \pi i} \int_{-i \infty}^{+i \infty} d s \frac{\Gamma\left(\frac{\Delta_{1}+\Delta_{2}}{2}-s\right) \Gamma\left(\frac{\Delta_{3}+\Delta_{4}}{2}-s\right) \Gamma\left(\frac{\Delta_{k}}{2}-s\right) \Gamma\left(\frac{\Delta_{4}-\Delta_{3}}{2}+s\right) \Gamma\left(\frac{\Delta_{1}-\Delta_{2}}{2}+s\right)}{\Gamma\left(\frac{\Delta_{1}+\Delta_{2}+\Delta_{3}+\Delta_{4}+\Delta_{k}-4}{2}-s\right)} \times \\
& \times \frac{(-s)_{m}\left(s+\frac{\Delta_{4}-\Delta_{3}}{2}\right)_{n}\left(s+\frac{\Delta_{1}-\Delta_{2}}{2}\right)_{n}}{\left(\frac{\Delta_{1}-\Delta_{2}-\Delta_{3}+\Delta_{4}}{2}+1\right)_{n}}{ }_{3} F_{2}\left(\frac{\frac{\Delta_{1}+\Delta_{2}+\Delta_{k}-4}{2}, \frac{\Delta_{3}+\Delta_{4}+\Delta_{k}-4}{2}, \frac{\Delta_{k}}{2}-s}{\frac{\Delta_{1}+\Delta_{2}+\Delta_{3}+\Delta_{4}+\Delta_{k}-4}{2}-s, \Delta_{k}-1} ; 1\right)
\end{aligned}
$$

where $(a)_{m} \equiv \frac{\Gamma(a+m)}{\Gamma(a)}$ is the Pochhammer symbol. Notice that the integral is convergent but we have not been able to compute it in closed form. ${ }^{88}$ Hence we have not been able to find the coefficients $d_{n}$ explicitly. However they can be computed, in principle, by solving equation (B.10). This can be done most efficiently by setting $v=1$ and considering (B.10) as an equation for two functions of $u$. The coefficients $d_{n}$ can then be determined order by order starting from matching the lowest order terms in $u$ and going up. Having determined the coefficients $d_{n}$ this way, we can then reconsider the dependence on $v$. The fact that the equation (B.10) remains true for all $u, v$, with the determined coefficients $d_{n}$, is a nontrivial result which depends on the fact that the Witten diagram has a consistent CPW expansion.

\section{Central charges in higher dimensional CFTs}

Two dimensional CFTs are characterized by a constant $c$ appearing in the most singular term of the $T \cdot T$ OPE, in the trace anomaly and also in the 1-point function of $T$ in a thermal background. We have

$$
\begin{aligned}
T_{z z}(z) \cdot T_{z z}(0) & =\frac{c}{2 z^{4}}+\frac{2 T(z)}{z^{2}}+\frac{\partial T_{z z}(0)}{z}+\ldots \\
T_{z \bar{z}} & =-\frac{c}{12} R \\
\left\langle T_{00}\right\rangle_{\beta} & =\frac{\pi}{6} \frac{c}{\beta^{2}}
\end{aligned}
$$

with $R$ the Ricci scalar of the manifold. The 1-point function in the last line is evaluated on $\mathbf{R} \times S^{1}$ at an inverse temperature $\beta$. Moreover, in a two-dimension QFT with coupling constants $g_{i}$ there exists a function $c\left(g_{i}\right)$ defined via the two point function of the stress tensor even away from a conformal point; the latter can be shown to flow monotonically along RG flows and to coincide with $c$ at fixed points in the flows $[112,113]$. This gives a very direct interpretation of $c$ as counting the available (massless, in the conformal case) degrees of freedom of the theory.

\footnotetext{
${ }^{88}$ One can close the contour to the right and pick up the residues from sequences of poles, as was done in [91], however we have not been able to evaluate the resulting infinite sums in closed form.
} 
In higher dimension, unfortunately, the story is not so straight forward. The analogues of the $c$ 's appearing in the three lines in eqn (C.1) are not generally equal. In four dimensions, for instance, several distinct quantities appear. The stress tensor has a canonical normalization fixed by the Ward identity

$$
\partial_{\mu}\left\langle T_{\mu \nu}(x) \phi\left(x_{1}\right) \ldots \phi\left(x_{n}\right)\right\rangle=\sum_{i} \delta\left(x-x_{i}\right)\left\langle\phi\left(x_{1}\right) \ldots \partial_{\nu} \phi\left(x_{i}\right) \ldots \phi\left(x_{n}\right)\right\rangle
$$

With this normalization the two point function takes the following form

$$
\left\langle T_{\mu \nu}(x) T_{\rho \sigma}(0)\right\rangle=C_{T} f_{\mu \nu \rho \sigma}(x)
$$

where $f_{\mu \nu \rho \sigma}(x)$ is a four-tensor fixed by conformal invariance and independent of the details of the CFT (for the exact form see e.g. [65]).

If the conformal field theory is placed on a curved manifold scale invariance is broken by the curvature of the manifold and this is reflected in the non-vanishing VEV of $T_{\mu}^{\mu}$ (which becomes $T_{z \bar{z}}$ in $2 \mathrm{~d}$ )

$$
\left\langle T_{\mu}^{\mu}\right\rangle=\frac{c}{16 \pi^{2}}(\text { Weyl })^{2}-\frac{a}{16 \pi^{2}}(\text { Euler })
$$

where the two terms correspond to the square of the Weyl tensor and the Euler density (see [65] for more details). Note that unlike the two dimensional case the trace anomaly is characterized by two constants, $c$ and $a$, rather than one. Of these two, $a$ is considered a candidate for a higher-dimensional analog to Zamolodchikov's two-dimensional $c$-theorem. In supersymmetric theories this result has even been partially demonstrated [114] using the fact that these coefficients are related via anomaly computations to the R-symmetry charges. It can be shown that the coefficients $c$ and $C_{T}$ are proportional [65].

Other interesting constants appear in the 3-point function of stress energy tensors. In two dimensions the 3-point function is proportional to $c$. In four dimensions the form of the 3 -point function is fixed by conformal invariance up to 3 constants $A, B, C$ [65]. A linear combination of these constants is proportional to $C_{T}$ (and thus to the anomaly $c$ ). Another linear combination is proportional to the anomaly $a$. The third linear combination is an independent quantity.

Finally if we formulate the CFT at finite temperature then, as discussed in the paper, operators can admit a temperature-dependent VEV and conformal invariance constrains the energy density to be of the form

$$
\left\langle T_{00}\right\rangle \sim \frac{\tilde{c}}{\beta^{d}}
$$

where $\tilde{c}$ is related by standard thermodynamic arguments to the entropy density. Note that, in contrast with the two-dimensional case, ${ }^{89}$ the constant $\tilde{c}$ is not protected and, in known examples, varies as a function of the CFT moduli. In $\mathcal{N}=4 \mathrm{SYM}$ at large $N$, for instance, it is known to vary by a factor of $3 / 4$ as the theory goes from weak to strong coupling.

\footnotetext{
${ }^{89}$ Where $\tilde{c}=c$ characterizes the CFT.
} 
Thus in a general $4 \mathrm{~d}$ CFT we have the following a priori independent constants: $c, a, \widetilde{c}$, as well as the undetermined linear combination of the $A, B, C$ constants in the 3-point function. However, as we argue in the paper, there is reason to believe that as one is sent to infinity the others must scale in essentially the same way ${ }^{90}$ (particularly $C_{T}$ and $\tilde{c}$ with which we are mostly concerned).

Open Access. This article is distributed under the terms of the Creative Commons Attribution License which permits any use, distribution and reproduction in any medium, provided the original author(s) and source are credited.

\section{References}

[1] N. Seiberg, Emergent spacetime, hep-th/0601234 [INSPIRE].

[2] J.M. Maldacena, The large- $N$ limit of superconformal field theories and supergravity, Adv. Theor. Math. Phys. 2 (1998) 231 [Int. J. Theor. Phys. 38 (1999) 1113] [hep-th/9711200] [INSPIRE].

[3] S. Gubser, I.R. Klebanov and A.M. Polyakov, Gauge theory correlators from noncritical string theory, Phys. Lett. B 428 (1998) 105 [hep-th/9802109] [INSPIRE].

[4] E. Witten, Anti-de Sitter space and holography, Adv. Theor. Math. Phys. 2 (1998) 253 [hep-th/9802150] [INSPIRE].

[5] T. Banks, M.R. Douglas, G.T. Horowitz and E.J. Martinec, AdS dynamics from conformal field theory, hep-th/9808016 [INSPIRE].

[6] V. Balasubramanian, S.B. Giddings and A.E. Lawrence, What do CFTs tell us about Anti-de Sitter space-times?, JHEP 03 (1999) 001 [hep-th/9902052] [INSPIRE].

[7] M. Berkooz and H.L. Verlinde, Matrix theory, AdS/CFT and Higgs-Coulomb equivalence, JHEP 11 (1999) 037 [hep-th/9907100] [INSPIRE].

[8] E. Witten, Spacetime reconstruction, talk at JHS 60 Conference, November 3-4, Caltech, U.S.A. (2001), http://quark.caltech.edu/jhs60/witten/1.html.

[9] D. Berenstein, Large-N BPS states and emergent quantum gravity, JHEP 01 (2006) 125 [hep-th/0507203] [INSPIRE].

[10] I. Heemskerk, J. Penedones, J. Polchinski and J. Sully, Holography from conformal field theory, JHEP 10 (2009) 079 [arXiv:0907.0151] [INSPIRE].

[11] I. Heemskerk and J. Sully, More holography from conformal field theory, JHEP 09 (2010) 099 [arXiv: 1006.0976] [INSPIRE].

[12] A.L. Fitzpatrick, E. Katz, D. Poland and D. Simmons-Duffin, Effective conformal theory and the flat-space limit of AdS, JHEP 07 (2011) 023 [arXiv:1007.2412] [INSPIRE].

[13] J. Penedones, Writing CFT correlation functions as AdS scattering amplitudes, JHEP 03 (2011) 025 [arXiv: 1011.1485] [INSPIRE].

[14] E.P. Verlinde, On the origin of gravity and the laws of newton, JHEP 04 (2011) 029 [arXiv:1001.0785] [INSPIRE].

\footnotetext{
${ }^{90}$ The relative scaling of $a, c, A, B, C$, and $C_{T}$ in the limit $C_{T} \rightarrow \infty$ follows, to some extent, from the inequalities derived in [115].
} 
[15] M. Van Raamsdonk, Comments on quantum gravity and entanglement, arXiv:0907.2939 [INSPIRE].

[16] M. Van Raamsdonk, Building up spacetime with quantum entanglement, Gen. Rel. Grav. 42 (2010) 2323 [arXiv: 1005.3035] [INSPIRE].

[17] R. Jost, The general theory of quantized fields, American Mathematical Society, Providence U.S.A. (1965).

[18] K.-H. Rehren, Algebraic holography, Annales Henri Poincaré 1 (2000) 607 [hep-th/9905179] [INSPIRE].

[19] M. Duetsch and K.-H. Rehren, Generalized free fields and the AdS-CFT correspondence, Annales Henri Poincaré 4 (2003) 613 [math-ph/0209035].

[20] R. Gopakumar, From free fields to AdS, Phys. Rev. D 70 (2004) 025009 [hep-th/0308184] [INSPIRE].

[21] R. Gopakumar, From free fields to AdS. 2, Phys. Rev. D 70 (2004) 025010 [hep-th/0402063] [INSPIRE].

[22] R. Gopakumar, Free field theory as a string theory?, Comptes Rendus Physique 5 (2004) 1111 [hep-th/0409233] [INSPIRE].

[23] R. Gopakumar, From free fields to AdS: III, Phys. Rev. D 72 (2005) 066008 [hep-th/0504229] [INSPIRE].

[24] O. Aharony, J.R. David, R. Gopakumar, Z. Komargodski and S.S. Razamat, Comments on worldsheet theories dual to free large-N gauge theories, Phys. Rev. D 75 (2007) 106006 [hep-th/0703141] [INSPIRE].

[25] O. Aharony and Z. Komargodski, The space-time operator product expansion in string theory duals of field theories, JHEP 01 (2008) 064 [arXiv:0711.1174] [INSPIRE].

[26] I. Klebanov and A. Polyakov, AdS dual of the critical $O(N)$ vector model, Phys. Lett. B 550 (2002) 213 [hep-th/0210114] [INSPIRE].

[27] J.M. Maldacena, A. Strominger and E. Witten, Black hole entropy in M-theory, JHEP 12 (1997) 002 [hep-th/9711053] [INSPIRE].

[28] G. 't Hooft, A planar diagram theory for strong interactions, Nucl. Phys. B 72 (1974) 461 [INSPIRE].

[29] A.M. Polyakov, Gauge fields and space-time, Int. J. Mod. Phys. A 17S1 (2002) 119 [hep-th/0110196] [INSPIRE].

[30] B. Sundborg, The hagedorn transition, deconfinement and $N=4$ SYM theory, Nucl. Phys. B 573 (2000) 349 [hep-th/9908001] [INSPIRE].

[31] O. Aharony, J. Marsano, S. Minwalla, K. Papadodimas and M. Van Raamsdonk, The Hagedorn/deconfinement phase transition in weakly coupled large- $N$ gauge theories, Adv. Theor. Math. Phys. 8 (2004) 603 [hep-th/0310285] [INSPIRE].

[32] O. Aharony, O. Bergman, D.L. Jafferis and J. Maldacena, $N=6$ superconformal Chern-Simons-matter theories, M2-branes and their gravity duals, JHEP 10 (2008) 091 [arXiv:0806.1218] [INSPIRE].

[33] N. Drukker, M. Mariño and P. Putrov, From weak to strong coupling in ABJM theory, Commun. Math. Phys. 306 (2011) 511 [arXiv:1007.3837] [INSPIRE]. 
[34] S. Hawking and D.N. Page, Thermodynamics of black holes in Anti-de Sitter space, Commun. Math. Phys. 87 (1983) 577 [InSPIRE].

[35] O. Aharony, S.S. Gubser, J.M. Maldacena, H. Ooguri and Y. Oz, Large-N field theories, string theory and gravity, Phys. Rept. 323 (2000) 183 [hep-th/9905111] [INSPIRE].

[36] O. Lunin and S.D. Mathur, Correlation functions for $M^{N} / S(N)$ orbifolds, Commun. Math. Phys. 219 (2001) 399 [hep-th/0006196] [InSPIRE].

[37] O. Lunin and S.D. Mathur, Three point functions for $M^{N} / S(N)$ orbifolds with $N=4$ supersymmetry, Commun. Math. Phys. 227 (2002) 385 [hep-th/0103169] [INSPIRE].

[38] A. Pakman, L. Rastelli and S.S. Razamat, Diagrams for symmetric product orbifolds, JHEP 10 (2009) 034 [arXiv:0905.3448] [INSPIRE].

[39] J.M. Maldacena and L. Susskind, D-branes and fat black holes, Nucl. Phys. B 475 (1996) 679 [hep-th/9604042] [INSPIRE].

[40] M.A. Vasiliev, Higher spin gauge theories in four-dimensions, three-dimensions and two-dimensions, Int. J. Mod. Phys. D 5 (1996) 763 [hep-th/9611024] [INSPIRE].

[41] M.A. Vasiliev, Higher spin gauge theories: star product and AdS space, hep-th/9910096 [INSPIRE].

[42] M. Vasiliev, Nonlinear equations for symmetric massless higher spin fields in (A)dS(d), Phys. Lett. B 567 (2003) 139 [hep-th/0304049] [INSPIRE].

[43] E. Sezgin and P. Sundell, Massless higher spins and holography, Nucl. Phys. B 644 (2002) 303 [Erratum ibid. B 660 (2003) 403] [hep-th/0205131] [INSPIRE].

[44] A.C. Petkou, Evaluating the AdS dual of the critical $O(N)$ vector model, JHEP 03 (2003) 049 [hep-th/0302063] [INSPIRE].

[45] R.G. Leigh and A.C. Petkou, Holography of the $N=1$ higher spin theory on AdS , JHEP 06 (2003) 011 [hep-th/0304217] [INSPIRE].

[46] S.R. Das and A. Jevicki, Large- $N$ collective fields and holography, Phys. Rev. D 68 (2003) 044011 [hep-th/0304093] [INSPIRE].

[47] R. de Mello Koch, A. Jevicki, K. Jin and J.P. Rodrigues, $A d S_{4} / C F T_{3}$ construction from collective fields, Phys. Rev. D 83 (2011) 025006 [arXiv: 1008.0633] [InSPIRE].

[48] M.R. Douglas, L. Mazzucato and S.S. Razamat, Holographic dual of free field theory, Phys. Rev. D 83 (2011) 071701 [arXiv:1011.4926] [INSPIRE].

[49] S. Giombi and X. Yin, Higher spin gauge theory and holography: the three-point functions, JHEP 09 (2010) 115 [arXiv:0912.3462] [INSPIRE].

[50] M.R. Gaberdiel and R. Gopakumar, An AdS $S_{3}$ dual for minimal model CFTs, Phys. Rev. D 83 (2011) 066007 [arXiv:1011.2986] [INSPIRE].

[51] M.R. Gaberdiel and T. Hartman, Symmetries of holographic minimal models, JHEP 05 (2011) 031 [arXiv:1101.2910] [InSPIRE].

[52] R. Minasian, G.W. Moore and D. Tsimpis, Calabi-Yau black holes and $(0,4) \sigma$-models, Commun. Math. Phys. 209 (2000) 325 [hep-th/9904217] [InSPIRE].

[53] J. de Boer, S. El-Showk, I. Messamah and D. Van den Bleeken, A bound on the entropy of supergravity?, JHEP 02 (2010) 062 [arXiv:0906.0011] [INSPIRE]. 
[54] J. de Boer, F. Denef, S. El-Showk, I. Messamah and D. Van den Bleeken, Black hole bound states in $A d S_{3} \times S^{2}$, JHEP 11 (2008) 050 [arXiv:0802.2257] [INSPIRE].

[55] E. Kiritsis, Product CFTs, gravitational cloning, massive gravitons and the space of gravitational duals, JHEP 11 (2006) 049 [hep-th/0608088] [INSPIRE].

[56] O. Aharony, A.B. Clark and A. Karch, The CFT/AdS correspondence, massive gravitons and a connectivity index conjecture, Phys. Rev. D 74 (2006) 086006 [hep-th/0608089] [INSPIRE].

[57] E. Kiritsis and V. Niarchos, (Multi)matrix models and interacting clones of Liouville gravity, JHEP 08 (2008) 044 [arXiv: 0805.4234] [INSPIRE].

[58] E. Kiritsis and V. Niarchos, Interacting string multi-verses and holographic instabilities of massive gravity, Nucl. Phys. B 812 (2009) 488 [arXiv:0808.3410] [INSPIRE].

[59] V. Niarchos, Multi-string theories, massive gravity and the AdS/CFT correspondence, Fortsch. Phys. 57 (2009) 646 [arXiv:0901.2108] [INSPIRE].

[60] E. Kiritsis and V. Niarchos, Large- $N$ limits of $2 D$ CFTs, quivers and $A d S_{3}$ duals, JHEP 04 (2011) 113 [arXiv:1011.5900] [InSPIRE].

[61] I. Bakas and E. Kiritsis, Bosonic realization of a universal $W$ algebra and $Z(\infty)$ parafermions, Nucl. Phys. B 343 (1990) 185 [Erratum ibid. B 350 (1991) 512] [INSPIRE].

[62] I. Bakas and E. Kiritsis, Grassmannian coset models and unitary representations of $W(\infty)$, Mod. Phys. Lett. A 5 (1990) 2039 [InSPIRE].

[63] I. Bakas and E. Kiritsis, Beyond the large-N limit: nonlinear $W(\infty)$ as symmetry of the $\mathrm{SL}(2, R) / \mathrm{U}(1)$ coset model, Int. J. Mod. Phys. A 7S1A (1992) 55 [hep-th/9109029] [INSPIRE].

[64] I. Bakas and E. Kiritsis, Target space description of $W(\infty)$ symmetry in coset models, Phys. Lett. B 301 (1993) 49 [hep-th/9211083] [INSPIRE].

[65] H. Osborn and A. Petkou, Implications of conformal invariance in field theories for general dimensions, Annals Phys. 231 (1994) 311 [hep-th/9307010] [INSPIRE].

[66] F. Dolan and H. Osborn, Conformal four point functions and the operator product expansion, Nucl. Phys. B 599 (2001) 459 [hep-th/0011040] [INSPIRE].

[67] F. Dolan and H. Osborn, Conformal partial waves and the operator product expansion, Nucl. Phys. B 678 (2004) 491 [hep-th/0309180] [INSPIRE].

[68] R. Rattazzi, V.S. Rychkov, E. Tonni and A. Vichi, Bounding scalar operator dimensions in 4D CFT, JHEP 12 (2008) 031 [arXiv:0807.0004] [INSPIRE].

[69] V.S. Rychkov and A. Vichi, Universal constraints on conformal operator dimensions, Phys. Rev. D 80 (2009) 045006 [arXiv:0905.2211] [INSPIRE].

[70] F. Caracciolo and V.S. Rychkov, Rigorous limits on the interaction strength in quantum field theory, Phys. Rev. D 81 (2010) 085037 [arXiv:0912.2726] [INSPIRE].

[71] D. Poland and D. Simmons-Duffin, Bounds on $4 D$ conformal and superconformal field theories, JHEP 05 (2011) 017 [arXiv: 1009.2087] [INSPIRE].

[72] R. Rattazzi, S. Rychkov and A. Vichi, Central charge bounds in $4 D$ conformal field theory, Phys. Rev. D 83 (2011) 046011 [arXiv:1009.2725] [INSPIRE]. 
[73] R. Rattazzi, S. Rychkov and A. Vichi, Bounds in $4 D$ conformal field theories with global symmetry, J. Phys. A 44 (2011) 035402 [arXiv: 1009.5985] [InSPIRE].

[74] A.C. Petkou and N.D. Vlachos, Finite size effects and operator product expansions in a CFT for $d>2$, Phys. Lett. B 446 (1999) 306 [hep-th/9803149] [INSPIRE].

[75] A.C. Petkou and N.D. Vlachos, Finite size and finite temperature effects in the conformally invariant $O(N)$ vector model for $2<d<4$, hep-th/9809096 [INSPIRE].

[76] E. Witten, Three-dimensional gravity revisited, arXiv:0706.3359 [INSPIRE].

[77] M.R. Gaberdiel, Constraints on extremal self-dual CFTs, JHEP 11 (2007) 087 [arXiv:0707.4073] [INSPIRE].

[78] M.R. Gaberdiel, S. Gukov, C.A. Keller, G.W. Moore and H. Ooguri, Extremal $N=(2,2)$ $2 D$ conformal field theories and constraints of modularity, Commun. Num. Theor. Phys. 2 (2008) 743 [arXiv: 0805.4216] [inSPIRE].

[79] S. Hellerman, A universal inequality for CFT and quantum gravity, JHEP 08 (2011) 130 [arXiv:0902.2790] [INSPIRE].

[80] S. Hellerman and C. Schmidt-Colinet, Bounds for state degeneracies in $2 D$ conformal field theory, JHEP 08 (2011) 127 [arXiv:1007.0756] [INSPIRE].

[81] A. Castro, A. Lepage-Jutier and A. Maloney, Higher spin theories in $A d S_{3}$ and a gravitational exclusion principle, JHEP 01 (2011) 142 [arXiv:1012.0598] [INSPIRE].

[82] H. Bloete, J.L. Cardy and M. Nightingale, Conformal invariance, the central charge and universal finite size amplitudes at criticality, Phys. Rev. Lett. 56 (1986) 742 [InSPIRE].

[83] P. Kovtun and A. Ritz, Black holes and universality classes of critical points, Phys. Rev. Lett. 100 (2008) 171606 [arXiv:0801.2785] [InSPIRE].

[84] L. Susskind and E. Witten, The holographic bound in Anti-de Sitter space, hep-th/9805114 [INSPIRE].

[85] J. de Boer, K. Papadodimas and E. Verlinde, Holographic neutron stars, JHEP 10 (2010) 020 [arXiv:0907.2695] [inSPIRE].

[86] X. Arsiwalla, J. de Boer, K. Papadodimas and E. Verlinde, Degenerate stars and gravitational collapse in AdS/CFT, JHEP 01 (2011) 144 [arXiv:1010.5784] [INSPIRE].

[87] I. Bena, On the construction of local fields in the bulk of $A d S_{5}$ and other spaces, Phys. Rev. D 62 (2000) 066007 [hep-th/9905186] [INSPIRE].

[88] A. Hamilton, D.N. Kabat, G. Lifschytz and D.A. Lowe, Local bulk operators in AdS/CFT: a boundary view of horizons and locality, Phys. Rev. D 73 (2006) 086003 [hep-th/0506118] [INSPIRE].

[89] A. Hamilton, D.N. Kabat, G. Lifschytz and D.A. Lowe, Holographic representation of local bulk operators, Phys. Rev. D 74 (2006) 066009 [hep-th/0606141] [INSPIRE].

[90] A. Hamilton, D.N. Kabat, G. Lifschytz and D.A. Lowe, Local bulk operators in AdS/CFT: a holographic description of the black hole interior, Phys. Rev. D 75 (2007) 106001 [Erratum ibid. D 75 (2007) 129902] [hep-th/0612053] [INSPIRE].

[91] H. Liu, Scattering in Anti-de Sitter space and operator product expansion, Phys. Rev. D 60 (1999) 106005 [hep-th/9811152] [INSPIRE]. 
[92] H. Liu and A.A. Tseytlin, On four point functions in the CFT/AdS correspondence, Phys. Rev. D 59 (1999) 086002 [hep-th/9807097] [INSPIRE].

[93] D.Z. Freedman, S.D. Mathur, A. Matusis and L. Rastelli, Comments on 4 point functions in the CFT/AdS correspondence, Phys. Lett. B 452 (1999) 61 [hep-th/9808006] [INSPIRE].

[94] E. D'Hoker, D.Z. Freedman, S.D. Mathur, A. Matusis and L. Rastelli, Graviton exchange and complete four point functions in the AdS/CFT correspondence, Nucl. Phys. B 562 (1999) 353 [hep-th/9903196] [INSPIRE].

[95] E. D'Hoker, D.Z. Freedman and L. Rastelli, AdS/CFT four point functions: how to succeed at $z$ integrals without really trying, Nucl. Phys. B 562 (1999) 395 [hep-th/9905049] [INSPIRE].

[96] E. D'Hoker, S.D. Mathur, A. Matusis and L. Rastelli, The operator product expansion of $N=4 S Y M$ and the 4 point functions of supergravity, Nucl. Phys. B 589 (2000) 38 [hep-th/9911222] [INSPIRE].

[97] L. Hoffmann, A.C. Petkou and W. Rühl, A note on the analyticity of AdS scalar exchange graphs in the crossed channel, Phys. Lett. B 478 (2000) 320 [hep-th/0002025] [INSPIRE].

[98] L. Hoffmann, A.C. Petkou and W. Rühl, Aspects of the conformal operator product expansion in AdS/CFT correspondence, Adv. Theor. Math. Phys. 4 (2002) 571 [hep-th/0002154] [INSPIRE].

[99] D.E. Diaz and H. Dorn, On the AdS higher spin $/ O(N)$ vector model correspondence: degeneracy of the holographic image, JHEP 07 (2006) 022 [hep-th/0603084] [INSPIRE].

[100] J. Polchinski, S matrices from AdS space-time, hep-th/9901076 [INSPIRE].

[101] S.B. Giddings, Flat space scattering and bulk locality in the AdS/CFT correspondence, Phys. Rev. D 61 (2000) 106008 [hep-th/9907129] [INSPIRE].

[102] M. Gary, S.B. Giddings and J. Penedones, Local bulk S-matrix elements and CFT singularities, Phys. Rev. D 80 (2009) 085005 [arXiv:0903.4437] [INSPIRE].

[103] M. Gary and S.B. Giddings, The flat space S-matrix from the AdS/CFT correspondence?, Phys. Rev. D 80 (2009) 046008 [arXiv:0904.3544] [inSPIRE].

[104] A. Polyakov, Nonhamiltonian approach to conformal quantum field theory, Zh. Eksp. Teor. Fiz. 66 (1974) 23 [INSPIRE].

[105] E. Witten, Anti-de Sitter space, thermal phase transition and confinement in gauge theories, Adv. Theor. Math. Phys. 2 (1998) 505 [hep-th/9803131] [INSPIRE].

[106] G. Festuccia and H. Liu, The arrow of time, black holes and quantum mixing of large- $N$ Yang-Mills theories, JHEP 12 (2007) 027 [hep-th/0611098] [INSPIRE].

[107] D.M. Hofman, Higher derivative gravity, causality and positivity of energy in a UV complete QFT, Nucl. Phys. B $\mathbf{8 2 3}$ (2009) 174 [arXiv:0907.1625] [INSPIRE].

[108] J. de Boer, M. Kulaxizi and A. Parnachev, $A d S_{7} / C F T_{6}$, Gauss-Bonnet gravity and viscosity bound, JHEP 03 (2010) 087 [arXiv:0910.5347] [INSPIRE].

[109] J. de Boer, M. Kulaxizi and A. Parnachev, Holographic Lovelock gravities and black holes, JHEP 06 (2010) 008 [arXiv:0912.1877] [INSPIRE].

[110] M. Kulaxizi and A. Parnachev, Energy flux positivity and unitarity in CFTs, Phys. Rev. Lett. 106 (2011) 011601 [arXiv: 1007.0553] [INSPIRE]. 
[111] S. Raju, BCFW for Witten diagrams, Phys. Rev. Lett. 106 (2011) 091601 [arXiv: 1011.0780] [INSPIRE].

[112] A. Zamolodchikov, Renormalization group and perturbation theory near fixed points in two-dimensional field theory, Sov. J. Nucl. Phys. 46 (1987) 1090 [Yad. Fiz. 46 (1987) 1819] [INSPIRE].

[113] A. Zamolodchikov, Irreversibility of the flux of the renormalization group in a $2 D$ field theory, JETP Lett. 43 (1986) 730 [Pisma Zh. Eksp. Teor. Fiz. 43 (1986) 565] [InSPIRE].

[114] K.A. Intriligator and B. Wecht, The exact superconformal R-symmetry maximizes a, Nucl. Phys. B 667 (2003) 183 [hep-th/0304128] [INSPIRE].

[115] D.M. Hofman and J. Maldacena, Conformal collider physics: energy and charge correlations, JHEP 05 (2008) 012 [arXiv: 0803.1467] [INSPIRE]. 